Portland State University

PDXScholar

Spring 6-3-2014

\title{
A Girl Power Study: Looking and Listening to the Role of Emotions and Relationality in Developing Critical Consciousness
}

Jennifer Wallin-Ruschman

Portland State University

Follow this and additional works at: https://pdxscholar.library.pdx.edu/open_access_etds

Part of the Cognitive Psychology Commons, and the Philosophy of Mind Commons Let us know how access to this document benefits you.

Recommended Citation

Wallin-Ruschman, Jennifer, "A Girl Power Study: Looking and Listening to the Role of Emotions and Relationality in Developing Critical Consciousness" (2014). Dissertations and Theses. Paper 1837. https://doi.org/10.15760/etd.1836

This Dissertation is brought to you for free and open access. It has been accepted for inclusion in Dissertations and Theses by an authorized administrator of PDXScholar. Please contact us if we can make this document more accessible: pdxscholar@pdx.edu. 
A Girl Power Study: Looking and Listening to the Role of

Emotions and Relationality in Developing Critical Consciousness

by

Jennifer Wallin-Ruschman

A dissertation submitted in partial fulfillment of the requirements for the degree of

Doctor of Philosophy

in

Applied Psychology

Dissertation Committee:

Janice Haaken, Chair

Eric Mankowski

Yves Labissiere

David Morgan

Robert Liebman

Portland State University

2014 


\begin{abstract}
The concept of critical consciousness centers on the capacity for involvement in social change efforts. Its development has been the aim of many recent social movements (e.g., the consciousness raising groups of the women's movement). In this work, critical consciousness is defined as the highest level of socio-political-cultural (SPC) consciousness development. SPC consciousness is characterized by the linking of the personal and the political so that structures and discourses of oppression are not only understood but also lead to critical action and transforming relations of domination. Additionally, critical consciousness includes the ability to tolerate ambivalence and conflict as well as the capacity to form group identifications that support critical reflection. While critical consciousness can develop in a variety of settings, it has a historical affinity with liberation education projects, particularly education projects that combine Critical Pedagogy and community engaged learning.
\end{abstract}

Empirical inquiry on critical consciousness development is extremely limited. This dissertation addresses that gap, focusing specifically on the role of emotion and relationality in critical consciousness development. Further, the study offers a feminist critique of the literature, addressing as well the contribution of Community Psychology to conceptualizing critical consciousness.

This dissertation analyzes data gathered through the Girl Power Senior Capstone, a course routinely taught at an urban Pacific Northwest public university. The six-hour course last for one quarter-term and integrates classroom time with community engagement. A central aim of the course is the development of critical consciousness. 
Specifically, the research was designed to address the following questions: 1) How are emotionally and relationally significant Girl Power experiences related to SPC consciousness development? 2) What tensions arise between the dominant culture and/or significant others' values and the values of the Girl Power capstone and how do these tensions move individuals toward or away from critical consciousness?

The theoretical framework and interview schedule were guided by participant observation of the Girl Power course conducted over an academic term. Semi-structured interviews were conducted with all consenting and available capstone participants $(\mathrm{N}=10)$ in the course where participant-observations were carried out. The interviews were transcribed and analyzed based on a modified version of Carol Gilligan's Listening Guide.

Two primary themes emerged from the data analysis-- the processes of awakening and sources of dissonance. The first theme relates to the processes of transformation that participants undergo during and following the course. Participants discuss this process as coming to see the world in a new way though their emotional experiences and relations developed in the course. The second theme, sources of dissonance, addresses sources of conflict that emerge as participants undergo this processes of awakening. Areas of tension that were particularly salient centered on relationships and experiences in the course. Participants identified experiences in the course that they perceived as contributing in key respects to SPC consciousness. Yet some aspects of change in the course seemed to reflect limiting capacities, including magical thinking, a limited range of critical action strategies, and lack of critical community post Girl Power. The findings 
from the dissertation can be used to inform the creation and implementation of future projects of critical consciousness development and social justice work more broadly. 


\section{Acknowledgments}

The completion of this document and degree were made possible by a fountain of social, emotional, and intellectual support. I am particularly indebted to my advisor, Jan Haaken, for her patience and guidance over the past six years. She has consistently pushed my writing and thinking to be more complex. The skills I have learned under her mentorship will continue to influence my academic and activist work. Eric Mankowski has also provided ongoing support that has helped me learn and grow as a scholar and person. Bob Liebman, Yves Labissiere, and David Morgan have completed the best committee that any student could ask for. The insights and support offered by each of my committee members have expanded this study and my skills into exciting and meaningful terrain.

I am also indebted to everyone involved in the Girl Power course. The opportunity to be a part of the beloved community created in the course not only allowed this study to be possible but also facilitated my own critical consciousness development. I am particularly indebted to the participants that took the time out of their busy lives to share their stories with me in interviews. I have learned a great deal from each of you.

I owe my sincerest gratitude and thanks to my friends and graduate student colleagues who have walked this long road with me. This document would not exist without the ideas, support, and comma control of Kealani Balfour, Mazna Patka, Sarah Van Dyke, Aisha Taylor, Diana Rempe, Jimena Alvarado and many others. 
Finally, my editor extraordinar and partner, Matt. I love you and could not have done this without you. 


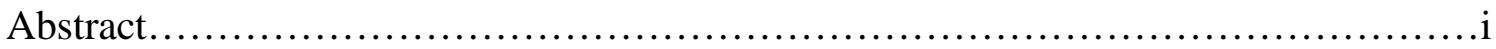

Acknowledgments...........................................................

Chapter One: Introduction..................................................

Chapter Two: Literature Review........................................... 32

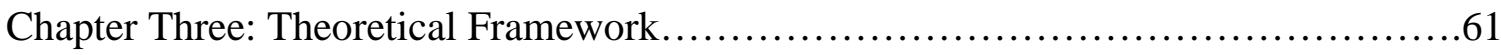

Chapter Four: Research Methods.............................................99

Chapter Five: Findings.................................................. 121

Chapter Six: Discussion................................................... 180

Chapter Seven: Conclusion...............................................213

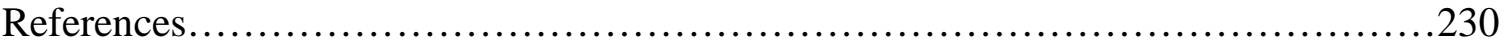

Appendix A - Interview Guide.............................................240

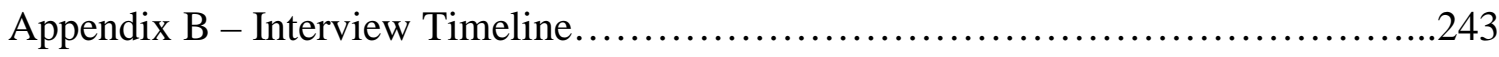

Appendix C - Smith's Conscientization Coding Categories Outline...................244

Appendix D - Watts \& Abdul-Adil Five Stage Model of Sociopolitical Development.247

Appendix E - Sample Girl Power Zine Page...................................248

Appendix F - Informed Consent Document.................................249 


\section{Chapter One: Introduction}

Critical consciousness is central to progressive social change. Social movement organizers and scholars acknowledge the importance of developing capacities for critique in a range of projects oriented toward social transformation (see Freeman, 1973; Gamson, 1992). Academics committed to action view the development of such capacities as a core component of their work (see Burton \& Kagen, 2005; Brydon-Miller, 2001; Moane, 2010; Montero, 2009; Prilleltensky, 2008). For the purposes of this dissertation critical consciousness is defined as a state of socio-political-cultural (SPC) consciousness development. It is characterized by the linking of the personal and political so that the structures and discourses of oppression are not only understood but the subject of critical action aimed at transforming relations of domination (Ife, 2001). Additionally, critical consciousness includes the ability to tolerate ambivalence and conflict as well as the ability to form group identifications that support critical reflection (Haaken, WallinRuschman, \& Patange, 2012). Educators committed to social justice have developed a range of curricula and teaching strategies specifically designed to promote critical analytic skills (see Freire, 2000; hooks 2994; Lynn, Benigno, Williams, Park, \& Mitchell, 2006).

As the women's movement was stressing consciousness-raising starting in the 1960's in the United States (see Ferree \& Hess, 2000; Freeman, 1973; Staggenborg, 2001), Paulo Freire was working in Brazil to develop a style of education focused on developing individual consciousness of sociopolitical structures and capacities to act against these configurations. Critical consciousness and consciousness-raising share a 
commitment to linking the personal to the political (Moane, 2010). However, the empirical understanding of critical consciousness development and consciousness-raising is stunted. Important questions remain under developed or unanswered. For example, what exactly is critical consciousness? How do we recognize it when we see it? What does the process of development look like? In addition, how do we, in fact, go about developing it? While some of these questions have been addressed in empirical literature, there are vast gaps in academic understandings of critical consciousness. This gap is perhaps widest in psychology, a field well suited to the study of critical consciousness. The dissertation situates itself in these fissures and expands the understanding of critical consciousness into new psychological territory.

\section{Why Critical Consciousness? Why Now?}

We live in a time of contradictions. Americans are quite politically disengaged; yet, civil unrest exists on both sides of the political spectrum, as represented by the Tea Party and Occupy movements. Voting levels are quite low and despite the visibility of protests, there is evidence to support the fact that most people have never engaged in this type of civic behavior (see Cornelius, 1998). Overall, it seems that only about half of the population votes and a much smaller percentage are involved in other types of civic engagement (e.g., direct action or advocacy). At the same time, many individuals do engage in some form of service-oriented work. Generally, this service often takes the form of charity, meaning that it is seen as a one way giving of resources from the provider to the "needy" recipient. While charity can help individuals, at least temporarily, it does nothing to address the root causes of the problems that put people in 
need of help in the first place. Further, engagement in charity alone does not appear to facilitate other citizenship behaviors (Cutforth, Stoecker, Marullo, Donohue, \& Strand, 2006). The need for engaged and informed citizens is assumed as a basis for a well functioning democracy. However, what counts as good citizenship behaviors is debated. I argue that engaged and informed citizens are those that possess the cognitive, emotional, and behavioral characteristics of a critically conscious individual.

Different conceptualizations of what good citizenship entails further complicate the picture of civic engagement. Some scholars and service providers argue for a conservative view of citizenship, which includes only engagement in voting behavior and/or charity based service (see Diemer \& Li, 2011). However, this limited definition leaves out a number of other behaviors necessary for a well-functioning democratic society. Kahne and Westheimer (1996) offer an expanded view and argue, "Citizenship in a democratic community requires more than kindness and decency; it requires engagement in complex social and institutional endeavors...Citizenship requires that individuals work to create, evaluate, criticize, and change public institutions and programs" (cited in Cutforth et al., 2006, p. 134).

Stefano Passini and Davide Morselli (2011) take a social psychological view of citizenship. They suggest individuals whom are supportive of democracy are also the same individuals that protest the government, suggesting a complex mental capacity and framing of some citizens. Passini and Morselli (2011) suggest protests and acts of civil disobedience may strengthen democracy. Using the classic social psychological problem of obedience and associated crimes of obedience (i.e., committing morally objectionable 
acts because of an authority) to argue disobedience has a progressive side. The authors suggest disobedience is an essential component of citizenship. If a democratic government leans toward authoritarianism, it is the responsibility of citizens to resist. Inherent in their claim is an important distinction: disobedience is not necessarily a positive for democracy but rather, is positive when enacted for legitimate demands. However, what is judged as legitimate depends on an individual's orientation to citizenship.

Passini and Morselli (2011) suggest three forms of citizenship identities: ruleoriented citizens who "see it as their task to follow the rules and respect authority's demands" (p. 258), role-oriented citizens who "perceive themselves as good citizens who meet their role obligations by actively obeying authority's demands" (p. 258), and valueoriented citizens who are "committed to the government because they share the cultural and institutional values on which they believe the state to be founded" (p. 259). It is this last group of citizens that tend to engage in acts of resistance and civil disobedience. These acts occur when citizens judge that authorities' are violating fundamental societal values. The authors conclude that facilitating the development of value-based citizens is one way to enhance democracy. Of course, different groups of citizens' hold divergent values so multiple groups of value-oriented citizens exist and often clash.

The importance of resistance and civil disobedience is not limited to citizens changing government and policy. They are central to youth working to counter regressive education systems and scholars working against oppressive and colonizing forms of research. The findings of my masters thesis (Wallin-Ruschman, 2011) 
correspond to those of Passini and Morselli in arguing that, resistance and disobedience are central to social change, and that can range from more regressive (e.g., simple rebellion against a single authority figure) to more progressive forms (e.g., possessing a complex understanding of social, political, and cultural hegemonic structures). The process of developing SPC consciousness acknowledges the range of resistance and disobedience in which an individual might engage.

In this dissertation, I view critical consciousness as the highest level of SPC consciousness development. SPC consciousness broadly represents the extent to which an individual can interpret broad social, political, and cultural structures and how these structures affect a person individually and groups to which a person may or may not belong. This aspect of SPC consciousness is captured in the common battle cry of the women's movement, "the personal is political." SPC consciousness also involves varying behaviors and emotions that change as individual move from one level of development to another. In chapter three, the Theoretical Framework, I lay out a full model of three states of SPC consciousness development and the three dimensions of each state--emotional, cognitive, and behavioral.

While working for critical consciousness is a stated goal of Liberation, Feminist, and Community psychologies (see Moane, 2010) the integration of these psychological subfields has been limited. The study offers a feminist critique of the critical consciousness literature, which has been shaped by a masculinist emphasis on rationality and individuality. By drawing on the work of feminist and social movement scholars, particularly those informed by psychoanalysis, I theorize the role of relationships and 
emotions in the process of SPC consciousness development. This critique expands the understanding of critical consciousness through the lens of women's experiences. In going beyond models based exclusively on cognitive processes, it also offers a more complete picture of the phenomenon. Critical consciousness can be understood through attending to emotional tensions involved in its development and maintenance and to the role dissonance plays in moving individuals along the SPC consciousness continuum.

Through dialogue and praxis, critical consciousness develops (Freire, 2000). Praxis is the process of acting on reality, reflecting on action, and then acting again in light of those reflections (Freire, 2000). While critical consciousness can develop in a variety of settings, it has a historical affinity with liberation education projects (see Freire, 1993; Moane, 2010), particularly education projects that combine Critical Pedagogy and community service-learning (Landreman et al., 2007; Reason et al., 2005). While individuals may be exposed to social problems and work for solutions in a variety of settings, I am grounding this dissertation in the field of education. The academic field of education currently and historically has been a place with the capacity to both create passive individuals who accept and recreate systems of oppression and active individuals who see through the veil of hegemony and fight systems of domination (Boler, $1997 \&$ 1999; Freire, 1993 \& 2000; hooks, 1994). This capacity to fight oppression arises out of the work of Freire, who serves as bedrock for the dissertation. In this study, I focus on an education project committed to social justice that carries the risk of recreating oppressive structures and the possibility of enhancing critical consciousness development. 
At least in the United States, many educational institutions are starting to take up the mantel of developing civically involved graduates (Checkoway, 2000). As suggested by the psychologist and education reformer, John Dewey, civic engagement and education are intimately related. Developing good citizens has often been considered the responsibility of educators (Dewey, 1999), but tension can arise when the definition of citizenship is expanded to include social justice behaviors and disobedience to authority. There is also disagreement over the methods best suited to the task of developing citizenship. Advocates of the expanded citizenship model argue one of the primary jobs of education is to prepare students to be actively engaged citizens that can interpret complex situations, think critically, and act to change unjust situations (Freire, 1993, 2000; hooks, 1994). Developing good citizens and offering students opportunities for service is important but not sufficient for engagement in social change. The development of individuals that can comprehend complex social problems and pursue social justice oriented goals in the face of seemingly insurmountable obstacles requires a range of psychological capacities associated with critical consciousness.

\section{Psychology and Critical Consciousness}

Psychology carries an important role in elucidating the mental and interpersonal processes involved in critical consciousness development. Psychological study of thoughts, emotions, and actions provides an ideal arena to investigate the three component parts of SPC consciousness. One of the ongoing issues in psychology concerns the disconnection between attitudes and behaviors. Within the research on SPC consciousness critical reflections and critical action are often severed, in which 
individuals develop critical reflection but often do not go on to engage in critical action (Watts, Diemer, \& Voight, 2011). Additionally, Psychology can help us understand the role that emotions play in critical consciousness development, an area of investigation that has been largely ignored within the critical consciousness literature. Martín-Baró (1994) goes so far to say "the fundamental horizon for psychology as a field of knowledge is concientizacion [critical consciousness]" (p. 39).

Community Psychology is particularly suited to take up the mantle of studying critical consciousness development. Further, the study of critical consciousness is an ideal location for psychological theory and research to contribute to social change. Community Psychology has historically been a change-focused field. This commitment to change ranges from its strong espousal of values such as social justice (Nelson \& Prilleltensky, 1997), an orientation towards more humanizing methods of inquiry and action (Prilleltensky, 1989; Rappaport, 1998), attending to multiple levels of analysis (Kelly, 1971), critiquing victim-blaming in research and policy (Albee, 1978; Ryan, 1971), and focusing on empowerment, liberation, and well-being (Prilleltensky \& Nelson, 2010; Rappaport, 1977). The study of critical consciousness has the potential to address each of these commitments. As a state, critical consciousness development is associated with building social justice allies (see Reason et al., 2005), contributing to empowerment, liberation, and well-being (see Prilleltensky, 2001), and fostering a systematic view that counters victim-blaming (Freire, 2000). Finally, as a process, critical consciousness is a key component of empowering methods, such as participatory action research (see Brydon-Miller, 2001). 
In a recent literature review on the topic, Roderick Watts, Matthew Diemer, and Adam Voight (2011) stated, "As a new area for U.S. social science, CC [critical consciousness] has not yet matured into well-articulated theory or a coherent body of empirical research" (p. 52). The findings of this dissertation begin to fill this gap, specifically in regards to emotion and process, and expand the study of critical consciousness in the broad field of Psychology and the specific field of Community Psychology.

\section{Critical Consciousness: An Overview}

Originally developed under the Portuguese term conscientização, critical consciousness refers to an individual's capacity to critically interpret their world and engage in individual and collective actions to change that world. Critical consciousness covers core aspects of psychology in that it is concerned with an individual's emotions, thoughts, and subsequent behaviors. What is lost in the English translation of the word is the focus on process. An alternative translation, consciousness-raising, captures Paulo Freire's focus on process rather than only a state of awareness. Currently, critical consciousness, much like the concept of empowerment, is viewed as both a state and a process (see Rappaport, 1987). This focus on process that leads me to use the language of SPC consciousness development, with critical consciousness being the most advanced state. To exclusively talk about critical consciousness as a state loses the understanding of the dynamic nature of the phenomenon. Therefore, at times I may talk about critical consciousness as a state to assist in understanding change over time. However, I 
primarily consider critical consciousness a developmental process through which individuals may move forward or backward on a continuum.

Critical consciousness has achieved some currency as an area of inquiry in Community, Liberation, Feminist, and Developmental psychologies. A number of models have been developed to study the component parts and/or stages in the development of critical consciousness (Carlson, Engegretson, \& Chamberlain, 2006; Diemer \& colleagues, 2007, 2009, 2010, and 2011; Guishard, 2009; Mustakova-Possardt, 1998; Smith, 1976; Wallerstein \& Sanchez-Merki, 1994; Watts \& colleagues, 1997, 1999, 2003, 2007, and 2011). Critical consciousness and the related phenomenon of socio-political development are not only useful for overcoming the individual and social effects of oppression but have been tied to general advances in critical thinking skills and individual well being (Watts \& Guessous, 2006). Further, any kind of involvement in social issues is thought to lead to increased levels of social competence and efficacy (Diemer \& Li, 2011). Diemer and Li (2011) cite several sources suggesting that critical consciousness "has been associated with urban African-American youth's mental health, school engagement among African-American and Puerto Rican youth, career development among low socioeconomic status youth of color, and occupational attainment in adulthood for low SES youth of color" (p. 1815). Increases in SPC consciousness benefit not only individuals but also society as a whole, as citizens become engaged and strive to change unjust aspects of the world.

I view SPC consciousness as existing at three broad states or levels. The first level of SPC consciousness development is pre-consciousness. As the name suggests, in 
this status there is no SPC consciousness. Overall, consciousness of consciousness is low and the understanding of the effect of internal and external factors on consciousness and behavior is even lower. The transitional level of SPC consciousness encompasses the widest variety of thoughts, emotions, and behaviors. It is at this state individuals begin to transition out of pre-consciousness and towards critical consciousness. The transformation is a highly emotional process because it involves significant changes to previous ways of knowing and being (Boler, 1999). It is during this level that individuals shed previous ways of thinking, feeling, and acting and replace them with new strategies-a highly emotional process. The highest state of SPC consciousness, critical consciousness, is characterized by critical action, critical efficacy, and critical reflection (Watts et al., 2011). Critical efficacy is the belief that one has the capacity to enact meaningful change. Critical action refers to actually being involved in transformational change. Critical reflection involves thinking about the world in a way that encompasses complexity and ambiguity, particularly around macro-social structures and their connection to individual level phenomenon. While I do take up this three-part model of critical consciousness, I argue that each component involves not only cognitions and behaviors but emotional dimensions as well. Elena Mustakova-Possardt (2004) says critical consciousness "is a precarious balance between mind and heart, where each serves as a corrective to the other, as a result of which the faculties of love, knowledge, and will function in relative unity" (p. 260). The quote suggests that critical consciousness is much more than cold cognition; it involves an essential and understudied emotional component, what she calls "heart." 


\section{Integrating Emotions into Critical Consciousness}

As with many other phenomena in the contemporary field of psychology, critical consciousness has been overwhelmingly studied as an exclusively cognitive phenomenon. Although cognition is important to SPC consciousness development, emotion is as well. For example, emotions as diverse as anger and love may be the stimulus that causes someone to have a critical moment of reflection that starts a journey towards critical consciousness. An emotion such as fear (as suggested by Freire, 2000) might be the roadblock preventing someone from engaging in critical action or serve as a stimulus for action.

To ignore the role of emotions in SPC consciousness is not only a result of the zeitgeist of the cognitive revolution in psychology but also a symptom of the historical neglect of emotions in scholarly work. Emotions have long been associated with the feminine, the illogical, and the weak (Boler, 1999). In the sciences, good academic work is generally associated with control over emotions (i.e., just the cold, hard facts). While bringing emotions back into academia is a worthy goal for many phenomena, it is particularly important to projects that claim to be counter-hegemonic in nature.

Megan Boler (1999) argues that, much like education, emotions are simultaneously a site of social control and critical resistance. In other words, emotions can hinder or enhance SPC consciousness development. However, the existing models of the emotional in critical consciousness are almost exclusively focused on the role emotions play early in the development of SPC consciousness (e.g., Carlson et al., 2006; Freire, 2000). Falling into the trap of considering emotions and logic as binary opposites, 
these models argue that, as SPC consciousness moves towards critical consciousness emotionality will become less important to behavior and thinking because of the increase in rationality and capacity for critical thinking. I disagree with this narrow view of emotion and suggest that it plays a role throughout SPC consciousness development and is important to all three-component parts of critical consciousness.

Scholars of social movements have suggested that emotions not only play a role in inhibiting involvement in social movements but also have the capacity to facilitate participation (Hogget, 2009; Jasper, 2011; \& Summers-Effler, 2002). Further, models of critical consciousness development that consider emotions as only important to the early phases of development fall victim to the binary splitting of emotion and reason. Models of this nature assume that as critical thought increases, emotions become less important to thinking and acting. I argue for a more nuanced and feminist view of emotion that suggests that emotion, cognitions, and behaviors are dynamically related. Further, emotions are not only central to cognition but also strengthen it, a view supported by the work of neurologist Antonio Damasio (1994). In other words, "people are smarter if they are animated by emotion as well as reason" (Clarke, Hoggett, \& Thompson, 2006, p. 6). This view is supported by a range of scholars that argue emotion and cognition are intertwined (see Damasio, 1994) and that emotion is intimately related to learning as suggested by Krathwohl's taxonomy of the affective domain in learning (Krathwohl, Bloom, \& Masia, 1964).

The language of emotion, feeling, and affect has been severely muddled in empirical literature and common parlance (Jasper, 2002). Paul Hoggett (2009) suggests 
the use of the term feeling as a broad category with two subsets, emotions and affect. Broadly, I define emotions as simultaneously biological and social, a view supported by Arlie Hochschild (1983). Emotions are based in a bodily or biological response that connects internal reality to the external world. Emotions are a sense (like touch or hearing) the help individuals understand the world. However, emotions are much more than a biological reaction. My view of emotions uses a social constructionist orientation to emotions. So although emotions are connected to the body how they are understood and interpreted depends on social context, which suggest an inherent connection between emotions and thoughts. Affect, on the other hand, is less connected to cognition and more directly connected to the body. Discussing the distinction, Hoggett (2009) says:

Feelings, which can be thought about and given a name - jealousy or spite for example - I regard as the emotions proper. But, many feelings are not like this we feel something, something has affected us, but it exists at the boundary of our consciousness. We feel something but we do not know what it is; it is more likely to affect us in our body, as a disturbance, than by accruing meaning to itself. This kind of feeling is more properly thought of as an affect. (p. 121)

In other words, emotion is more accessible to consciousness- although still connected to a biological response- it is something that can likely be labeled while feeling is more of an ephemeral bodily sensation. Feeling on the other hand is characterized as "the general area within which we make those distinctions along a spectrum of continuity between body and mind, individual and society, nature and culture" (Crociani-Windland \& Hoggett, 2012, p. 164). Affect, represents the end of this continuum that is more directly associated with the body. The social nature of emotions necessitates that the study of emotion include attending to the relation dimension as well. Emotions are constructed not just on an individual level but in social interactions. 
Within the level of emotions, I wish to make one further distinction using Randall Collins, who argues for emotion to be further broken down into transient emotions and emotional energy. The first, transient emotion, refers to relatively short term emotional experiences that are often labeled, such as joy, fear, and anger. Emotional energy, on the other hand, is "a more long term emotional tone that is durable from situation to situation" (Summers-Effler, 2002, p. 42).

Hoggett (2009) suggests that emotions involve an oscillation between feelings considered opposite. This oscillation occurs because of the ambivalence involved in the cognitively complex nature of some emotions, such as shame and pride. Hoggett argues that transient emotions are shifting in nature, occurring in "an intimate and dynamic relation to others—grief/grievance, shame/pride, love/hate, despair/hope" (p. 94). He goes on to argue that it is their labile nature that contributes to how emotions can influence efficacy. In chapter three, I introduce my interdisciplinary theory of the impact of emotions and the related concept of relationality on critical consciousness development.

\section{Critical Consciousness in the University Classroom}

Much of the previous psychological work on critical consciousness (Carlson et al., 2006; Diemer \& colleagues, 2007, 2009, 2010, and 2011; Guishard, 2009; WallinRuschman, 2011; Watts \& colleagues, 1997, 1999, 2003, 2007, and 2011) studies individuals within relatively homogeneous, usually marginalized groups. While this work is invaluable, it also is important to understand the effects of critical consciousness 
enhancing programs in a broader range of settings. The university classroom offered a variety of advantages for my dissertation.

First, there is considerable variability of individual experiences within the average classroom. Relatively little is known about how intersecting identities and histories result in different trajectories of SPC consciousness development, especially when being exposed to similar stimuli (Landreman et al., 2007). This is an important area of study because, based on what happens (or does not happen) in a setting, scholars and activists can better create interventions, which take the specific backgrounds and identities of those involved to facilitate a more ideal environment for the development of critical consciousness. Only by understanding such processes and factors associated with the variability of students' experiences can instructors design more optimal situations for critical consciousness development.

A second reason for the selection of the university classroom in studying critical consciousness involves the developmental stage of university students. They are basically fully cognitively developed (Piaget, 1953), are ending their stage of identity exploration (Erikson, 1968), and should still be undergoing moral development (Gilligan, 1982; Kohlberg, 2008). Even for students that are not in this developmental period, college courses offer an ideal environment to challenge personal and cultural assumptions that might go unnoticed in other domains (Landreman et al., 2007). Although much of the research on critical consciousness has focused on younger groups, it makes sense to study individuals that are more fully developed. 
Third, there are multitudes of projects aimed at increasing critical consciousness in educational settings. It is important to study as many of these settings as possible to understand unique dynamics that contribute to the development of critical consciousness. However, it cannot be assumed that these interventions always have positive results. Rushing blindly into any new technique can have unintended consequences for students and community members. For example, after service-learning was being utilized heavily in university classrooms it was found that sending students into historically disadvantaged communities without proper preparation could result in increased negative stereotypes (Cutforth et al., 2006). This was not only problematic for the development of the student but also potentially harmful to involved community members (Eyler, 2002).

\section{Setting: Portland State University's Senior Capstones}

In my search for a setting to study critical consciousness, I looked into the unique opportunities afforded by Portland State University's undergraduate curriculum, particularly the University Studies program. This innovative curriculum starts during students' freshman year when they take a yearlong Freshman Inquiry course, which introduces students to "different modes of inquiry" and provides "them with tools to succeed in advanced studies and their major" (UNST Introduction, n.d.). During the sophomore year students take three Sophomore Inquiry courses which "lead into a thematically linked, interdisciplinary cluster of courses at the upper-division level" (UNST Introduction, n.d.).

The culmination of the University Studies curriculum is the six-credit Senior Capstone course. Although there are a range of experiences offered under the capstone 
courses, all share the common goal of increasing community engagement, critical thinking, and supporting students as they act in a socially responsible way (UNST Introduction, n.d.). Further, the courses often utilize reflection and place a high value on community and student voice. For this dissertation, I worked with the assistant director of University Studies to find a capstone course that heavily integrates Critical and Feminist Pedagogies and community-based learning.

Research on Portland State University's senior capstone courses has shown that most students (e.g., $60 \%$ or above) felt that they would continue to volunteer after their capstone experience, that the capstone experience increased communication and problemsolving skills, and that the capstone increased awareness of social and ethical responsibility (Rhodes \& Agre-Kippenhan, 2004). Research on the capstone has also shown that "eight of ten students said they believed...individuals can do something to bring about change in society" (Rhodes \& Agre-Kippenhan, 2004, p. 5) suggesting an increase in critical efficacy.

In carrying out a dissertation in the field of Education, Stephanie Stokamer (2011) analyzed survey data on student evaluations of the capstone courses. The surveys ranged over a five-year period and looked at over 11,000 students in 700 courses. Stokamer identified those aspects of the capstone courses that had the strongest effect on civic competence. This study defined civic competence as the knowledge, skills, attitudes, and actions necessary for effective democratic participation. She found that the communitybased learning component of the course was not enough to facilitate civic competency and that the most effective courses utilized an increased focus on social justice and 
diversity issues. Further, the most effective pedagogical strategies for increasing civic competence included: utilizing class discussion, having a syllabus that directly connects class and community experiences and knowledge, discussing social justice issues, such as race and politics, and having an ongoing process of revising the course. Based on these findings Stokamer describes what she terms the Critical Pedagogy Model of Civic Competence. She argues that "critical pedagogy is necessary to most deeply and effectively help students understand community-based learning in the broader spectrum of civic participation for social change, to create space for dialogue around issues of privilege and difference, and to challenge systems of oppression (Kitano, 1997; Souza, 2007; Yep, 2011)" (cited in Stokamer, 2011, p. 7). These findings are foundational for this dissertation. Critical consciousness and civic competence are related phenomenon; both involve increasing knowledge of socio-political systems and processes, increasing levels of efficacy, commitment to some form of social action, and the understanding that social justice is important to democracy. Stokamer's findings suggest I looked in the right place (i.e., courses that utilize community engaged learning and Critical Pedagogy) to find high levels of SPC consciousness development. However, Stokamer's findings stay in the limited realm of civic competence, which as discussed above, does not adequately reflect the range of behaviors, emotions, and thoughts that could be considered desirable for a well functioning democracy. The goals of this dissertation were to expand this discussion to include the more progressive notion of critical consciousness, expand the study of critical consciousness into the emotional, and gain a 
preliminary understanding of how the capstone experience fits into the process of SPC consciousness development.

\section{Overview of Methods}

This dissertation is rooted in qualitative research methodology. The nature of the phenomena of interest is multidimensional, process oriented, and context specific, making qualitative research methods particularly applicable. This study is primarily situated in a constructivist interpretive paradigm that strives for trustworthiness, credibility, transferability, and confirmability (Denzin \& Lincoln, 2005). Within this paradigm, knowledge is co-constructed by the researcher and participant, and reality is a multiple and intersecting phenomena (Lincoln \& Guba, 2005). As Stein and Mankowski (2004) argue, qualitative research methods "embody the values of community research and action", allow for an enhanced understanding of "individual diversity and the nuance of social context", "enhance the study of behavior embedded in a larger social world", and aid in the development of "ecologically-sensitive constructs" (p. 21). Each of these advantages of qualitative research fit closely with the aims of this dissertation. Further, the use of qualitative methods adds not only to the analytic goals of this study, but also my commitment to action. Stein and Mankowski suggest that qualitative methods can aid in the process of enhancing voice to oppressed research participants but can also contribute to a process of analyzing hegemonic structures by dominant groups. While the full explication of my dissertation methods occurs in a later chapter, I briefly introduce them here. 
Preliminary Research. To better prepare for the primary form of data collection (i.e., interviews) I engaged in a process of preliminary research. This preliminary work involved the criterion selection (Patton, 2001) of a senior capstone course ideally designed to increase critical consciousness (i.e., utilized Critical Pedagogy and community based learning). Also, my focus on the emotional dimension of SPC consciousness development lead me to seek out a course that also utilized Feminist Pedagogy as feminist educators have more fully integrated emotions into their theory and teaching methodology than purely critical pedagogues.

After meeting with multiple members of the University Studies faculty, including the Assistant Director, I was pointed towards the Girl Power capstone - one of the longest running and most successful in the program - as an ideal place to observe my phenomenon of interest. The Girl Power capstone is a one quarter, six credit hour course. I then met with the Girl Power capstone instructor and we developed a plan for me to become a participant observer in the capstone. A description of the Girl Power capstone is presented in the Methodology and Findings chapters.

Participant observation is conceptualized as a method of data collection that allows the researcher to use her/his knowledge and experience in the setting to aid in the collection and analysis of events (Kidder \& Fine, 1997). Rochford (1985) states that a benefit of an active member role in research is the ability to grasp the "subjectively meaningful world of members, rather than the objective analytic accounts of their worlds" (cited in Adler \& Adler, 1987, p. 52). 
In my role as participant observer, I simultaneously acted as an observer by collecting field notes from the various aspects of the course and as a participant by engaging in the course. I was an active member (Adler \& Adler, 1987) of the course, engaging in most functional course activities but also consistently engaging in research as well. Although the full explication of my preliminary research is addressed in chapter four, I briefly note the advantages I gained from acting as a participant observer in the class. First, through my roles as an active member I developed rapport with participants I later interviewed (Adler \& Adler, 1987). During interviews, it seemed that participants' familiarity with me allowed them to open up and share stories that are more personal and struggles they experienced in regards to their course experience and critical consciousness development. Second, the preliminary research helped me to fine tune my theoretical framework and develop my methodology. The expression of emotions in the classroom (e.g., anger, joy, frustration) solidified the theory that emotions and relationality were central to the process of SPC consciousness development. In my time as participant observer, emotions were visible in the classroom lending further support to my theory of emotions as central to the development of critical consciousness. However, these observations do not tell me how or why emotions are an important component of the process. I realized that to get at this level of understanding of emotions and the associated course experiences a methodology that allows for more depth of probing was necessary. I thus integrated interviews into my analysis and made them the primary form of data collected for the dissertation. Further, observations from the course were used in combination with my developed theory to create the interview guide for the study. 
Third, through my role as a participant observer I was able to gain personal knowledge of the experience of the capstone. Instead of solely relaying on participant accounts of the experience I have a subjective experience to draw on, which allowed me to assess inconsistencies in thoughts or actions and use of the language the participants associate with the experience. Personal experience increases the level of credibility in qualitative research (Lincoln \& Guba, 1985). The intricacies of the Girl Power experience identified during interviews may have been lost on me if I had not participated in the course myself. Through my active role in Girl Power, I have a subjective understanding of what it means to take this capstone, because I have also been through the process. I feel this knowledge was essential to both interpreting the data but also served as essential when I constructed my representations of the participants.

Observations allowed me to go through the same process as the participants so that I could better understand and interpret the seminal experiences of the course. During my observations, I took extensive field notes, which included the class discussions, the discussions in one ongoing mentor groups, the students' bodily reactions during class, and my own feelings and reactions. These observations informed my methodology construction and aided in the analysis of other primary forms of data. Self-reflection and reflexivity are also essential to field research in an active member role. I have detailed my process of this in chapter six.

Sources of data. The primary form of data consisted of interviews conducted with all consenting and available capstone participants. These interviews were semistructured and focused around understanding participants' emotional and relational 
experiences in the course and areas of dissonance associated with the course. During the interviews I invited participants to help me theorize the processes involved in SPC consciousness development for themselves. Semi-structured interviews are considered ideal for phenomena that are highly complex (see Smith, 1995), such as emotions and critical consciousness.

Interviews were audio digitally recorded and most lasted for about one hour. In addition to recording the interviews, I took notes during the interview and spent time immediately after the interview writing reflections on the experience, specifically noting the participants' reactions throughout the interview and my personal responses to what was said. Questions for the interviews were primarily drawn from the theoretical framework (see chapter three), research questions, and my preliminary research. The final interview guide (see appendix A) was designed to look at a range of feelings related to the phenomenon by asking students not only how they thought or acted but also how they felt. The guide was also designed to help me utilize participants' insight in developing my theory of the emotional dimension of SPC consciousness development. I carried out a pilot of the interview guide with a past participant of the Girl Power capstone. My interview guide changed slightly following the feedback of the practice interview. For example, the practice interview subject stated that popular culture would be more accessible wording than dominant culture in my interview script, a change reflected in the wording of question nine. Additionally, after the first few interviews I met with my dissertation chair to review two of the first transcripts. Based on feedback from this meeting I made some additional, small, edits to the interview guide. 
Conducting interviews after the end of the course provided students the opportunity to have reflected on the class and experienced changes in emotions, behaviors, and/or attitudes. The instructor of the capstone suggested that students often resist the anti-hegemonic teachings of the course and rebel against this challenge to their traditional ways of viewing themselves and the world (a view supported by Cho \& Lewis, 2005). Rebellion may manifest during the capstone as a general dislike of the course and the instructor or as disengagement from the course. It has been the instructor's experience that many of the students who go through this period of dislike and/or disengagement later contact her to express how influential the experience was for them. Finally, conducting interviews after the completion of the course allowed time for me to develop rapport with the capstone students (Adler \& Adler, 1987). I feel that this rapport was truly essential to the success of my interviews as I was already aware of the participants past struggles, and they of mine, which facilitated an open discussion during the interviews.

Research suggests that individuals are more apt to remember emotionally evocative material (Haaken, 1998) suggesting that my strategy of retrospective interviews fits well with my focus on emotions. Additionally, these interviews allowed me the opportunity to visit any longer term changes to attitude, emotion, and behavior. Landreman and colleagues (2007) argue "Attempts to measure learning outcomes related to intergroup awareness or the development of critical consciousness at the time of program completion may miss learning outcomes that unfold more slowly" (p. 292). The 
present study represents a small step in the direction of a post-course completion investigation.

All interviews included a set of standard questions, which I constructed based on the research questions, theoretical framework, and preliminary research and edited based on my experience in the practice and early interviews. Semi-structured interviews are used to probe interesting and emergent points (Smith, 1995). I also included more personalized and projective aspects to the interviews. Hoggett (2009) suggests that affective experience is often non discursive, or unable to be articulated. Given this potential, I wanted to be creative in the multitude of ways that I elicited response from my participants in the interviews. When scheduling interviews with participants I asked them to bring artifacts from the course that they produced (e.g., weekly journals, Zine pages, secondary projects, reflections, and/or art). During the interviews, I prompted participants to talk about these artifacts and relate them to any experiences or emotions they had in the course. A portion of the participants complied with this request and brought objects. However, many did not- I was prepared for this possibility and asked students what they would have brought, which elicited answers similar in content and form to those participants that did bring a physical artifact.

I also utilized a timeline of significant course events in the interviews (see appendix B). In the first few interviews, I asked participants to track their emotional reactions throughout the listed course events. Some students struggled to do this so in later interviews I also asked participants to take a moment and write either feeling words or faces on the timeline and then talk me through the their emotions in the course. 
Interviews were transcribed with attention to pauses, inhalation and exhalation, changes in voice, laughing, and other potential markers of emotion. My field notes were triangulated with the results of the interview analysis and my personal experiences in the course used to aid in my interpretation of data. I contacted all female capstone participants $(\mathrm{N}=16)$ and 10 responded to my inquiries and were interviewed.

Data analysis. Dedoose, online qualitative data analysis software, was used to manage and code the interview transcripts. I analyzed the interviews using Carol Gilligan's Listening Guide (Gilligan, Spencer, Weinberg, \& Bertsch, 2003). The Listening Guide is a powerful tool for hearing the multiple layers of a narrative, so one interpretation or listening may bring out the participants literal meaning while another digs deeper to listen for how the speaker uses the first person or expresses emotion. Further, like the Girl Power capstone, the Listening Guide is based in a feminist lens of analysis including a focus on voice and relationships. The Listening Guide provided a way to listen to how participants' stories are dissonant or in harmony with one another. Additionally, I also listened to how students resolved these areas of dissonance and conflict. More details of my use of the Listening Guide are provided in chapter four.

Ethics and Participation. The Girl Power capstone was a unique and challenging research location. The capstone is an intimate and vulnerable environment with personal sharing occurring on an almost daily basis. I brought my own experiences and feelings into the daily course activities through personal sharing in whole class and small group discussion, which facilitated my role as a course participant. I am thankful I was accepted into the beloved community but I was constantly attending to my dual role as 
researcher and participant and worked to ensure the participants understood my research project and role in the class. This was achieved primarily by discussing the goals and methods of the project - I did this through a series of informal discussions throughout the quarter. On the first day of class, I introduced myself and talked about my project and the role students would play in the project. At two other points in the quarter, I gave students an update on my dissertation and talked with them about the potential ethical tensions of such a project. After each of these informal presentations, I answered student questions about the project, their role in the research, and the process of research and graduate school more broadly. Following the last two of these brief presentations, a handful of students stated that they forgot I was not a regular classroom participant and that I was conducing research. I saw this as a potentially problematic position and integrated this insight into the development of my ethical guidelines. However, I also viewed these comments as a reflection of successfully becoming a participant in the course activities, culture, and routine.

Through both its classroom and community intervention, the Girl Power capstone is an activist project that is focused on increasing women and girls' voices. The dual visions of voice and confidentiality make for a complex ethical situation that cannot be easily amended by any standard set of ethical guidelines. These dual goals set up a potential contradiction between valuing research participant voice and protecting confidentiality. To amend this possible incongruence I integrated capstone participants' opinions into the development of the informed consent process allowing participants to have a voice in amending this ethical conundrum. This decision draws on the work of 
Brinton Lykes (1989), who contends that informed consent processes in some cultural contexts can actually represent a barrier to working in a participatory fashion because they exist to release the university from liability while also maintaining control of research. Lykes (1989) further argues that when a consent form is introduced that has not been co-developed with research participants; it may alienate the participants and alter the relationship with the researcher. By integrating participant concerns and feedback into the informed consent document I hoped to avoid this fate and potentially increase the likelihood that capstone, students would agree to be a part of the interview process. I acknowledged that participants may not attend to all potential ethical considerations of the project and thus informed them of the reality that the Human Subjects Research Review Committee would make the final decision regarding the informed consent process for the study. Although, the process of consent developed by the course participants' and myself was approved. I also gave participants chances to review portions of the data. Capstone students were given the opportunity to review their interview transcripts and amend or delete any sensitive information. Participants were also provided with a summary of the research findings.

Research questions. This dissertation research was designed to address the following research questions:

1) How are emotionally and relationally significant Girl Power experiences related to SPC consciousness development? 
2) What tensions arise between the dominant culture and/or significant others' values and the values of the Girl Power capstone and how do these tensions move individuals toward or away from critical consciousness?

Findings. Two primary themes arose from my data analysis, the Processes of Awakening and Sources of Dissonance. The first theme connects to the first research question and relates to the processes of transformation that participants go through during and following the course. Participants discuss this process as coming to see the world in a new way though the emotional experiences, academic content, and relations developed in the course. These processes are not without tension, however, nor are they directly related to social action or engagement in activism. The second theme, Sources of Dissonance, relates to the second research question and addresses sources of conflict that emerge as participants undergo this process of awakening. Areas of tension that were particularly salient centered on relationships and experiences in the course. Each of the two themes is addressed in more detail in chapter five.

\section{Conclusion}

The following chapters lay out the dissertation in more detail. Chapter two, the Literature Review, has two main components. First, it addresses the concept of oppression, which is essential to understanding the concept of critical consciousness and why it is important. Second, I offer an historical overview of critical consciousness literature, attending to the gaps that exist around emotion and relationality. In chapter three, the Theoretical Framework, I address the emotional gap in the field of critical consciousness by utilizing theory from interdisciplinary scholars that study emotions. 
Using this additional theory I develop a new continuum of SPC consciousness development that integrates emotions throughout the process. Chapter four describes the methods and mode of analysis used to address the research questions, as well as discuss the ethical implications of the study. Chapter five describes the two primary themes that arose from my data analysis and provides representative data for each of the themes and subthemes. Chapter six addresses the theoretical implications of my findings. Chapter seven provides a brief overview of the study findings. This chapter also addresses the implication and limitations of this dissertation and offers future directions for this line of research. 


\section{Chapter Two: Literature Review}

I begin this chapter by addressing and defining the overarching concept of oppression. This provides a basic understanding of oppression, onto which I map my proposed solution, critical consciousness, detailed in the second section. The literature reviewed in this section is presented chronologically in order to demonstrate how this study fits into the line of research in this area. Throughout this chapter I focus on presenting past works that have influenced the current project. Chapter three takes the most relevant theories from this section and organizes them into a coherent theoretical framework and model for the current project. In this next chapter, I augment the gaps in past research with theories of affect and emotions from education, sociology, and psychoanalysis.

\section{Laying the Groundwork: Understanding and Defining Oppression}

To work towards progressive social change at the political and psychological levels requires an understanding of what must be altered. Oppression is a state and process that exists at the social and psychological levels. Attempts at research, education, and action aimed at alleviating oppression often ignore the complexity of the phenomenon and instead strive to address one component part of oppression. My frustration with one-dimensional attempts to fight oppression led me to my commitment to socio-political-cultural (SPC) consciousness development. As an activist, I work with others to fight unequal distribution of resources, including access to education. As a psychologist, I work to help empower people to change their situation. There has been a disjuncture, however, in these two aspects of my work. These two positions do not 
necessarily speak to one another and cannot adequately fight injustice because of the lack of an integrated understanding of oppression. Before a study of critical consciousness can be undertaken, a multifaceted understanding of oppression is necessary because you cannot know how to fight something that you do not understand.

Iris Young's Five Faces of Oppression. Young (1990) argues that while all oppressed people share a common "inhibition of their ability to develop and exercise their capacities and express their needs, thoughts, and feelings" (p. 40), the experience varies drastically from one group to another. These varied experiences lead to Young's conceptualization of oppression as having five faces: exploitation, marginalization, powerlessness, cultural imperialism, and violence. Young argues that oppression cannot be fully understood as existing solely in relation between two groups, one oppressed the other oppressing. Oppression also emanates from well meaning practices and unconscious tendencies through these five faces.

Exploitation. Young (1990) uses Marx's theory of exploitation to address the first face of oppression. Exploitation occurs "through a steady process of transfer of the results of the labor of one social group to benefit another" (p. 49). Systems are structured so the energy and work of the oppressed serve to benefit already more powerful groups through increasing their wealth, prestige, and/or power. Young expands Marx's theory of exploitation beyond class and argues exploitation occurs for women and racial minorities as well. For women exploitation occurs when their material labor and "nurturing and sexual energies" (p. 50) are transferred to men. By drawing on women's energies in these areas men are able to free up their own energy to pursue creative and career passions. 
For racial groups on the margins, exploitation occurs through their placement in menial labor, or jobs that serve whites.

Overcoming exploitation is a complex process. Young (1990) argues the transfer of resources alone cannot amend the process of exploitation. As long as the rules of the game are rigged in such a way to benefit some groups and not others, the unequal distribution of resources will be recreated. Instead, long lasting change would require "alteration of the division of labor, and similar measures of institutional, structural, and cultural change" (p. 53).

Marginalization. Young (1990) argues that marginalization is the most dangerous form of oppression and is becoming increasingly widespread. Marginalization occurs when certain social groups are kept out of the labor market, which results in severe resource deprivation in those groups. Those who are excluded from the work force are required to obtain their means of survival in other ways, often utilizing the welfare state. Depending on welfare means being subject to its dehumanizing processes, such as "to suspend basic rights to privacy, respect, and individual choice” (Young, 1990, p. 54). Further, not being allowed to participate in the work force can result in helplessness, low self-efficacy, and low self-esteem.

Powerlessness. Concerning class privilege, powerlessness involves not having the ability to exercise authority. Young (1990) explains that nonprofessionals (i.e., the working class) experience powerlessness because they lack opportunities for growth, autonomy, and respectability. Young suggests this last point, respectability, is intimately intertwined with race and gender. White men are generally automatically granted 
respectability while women and people of color are not. It is only through proving their credentials that men of color and women can gain a level of respect. Powerlessness exists when nonprofessional workers lack the luxury of self-directed work and are exposed to disrespectful treatment by those in positions that are more professional. In addition, nonprofessionals make less money and thus possess less material resources than professionals.

Cultural Imperialism. While the previous three faces of oppression are matters of “concrete power in relation to others" (Young, 1990, p. 58) cultural imperialism involves marking a group as simultaneously invisible and stereotyped. The dominant culture of upper class, white, men, is not only considered better but also normalized within society and popular culture, which is often controlled by this relatively small subset of the population. Any culture, which deviates from the dominant culture, is considered aberrant and generally pathologized. Stereotyping, discussed further in the next section, plays a key part in this process. By attaching labels, dominant culture limits the full expression of humanity of the stereotyped Other. Through cultural imperialism, the very experience of oppressed groups is denied. Their reality is considered false, this occurs through being made invisible but also marked as a deviant Other. Young (1990) summarizes the injustice of cultural imperialism as "that the oppressed group's own experiences and interpretations of social life finds little expression that touches the dominant culture, while that same culture imposes on the oppressed groups its experience and interpretation of social life" (p. 60). 
Violence. Violence becomes a form of oppression when it takes on a systematic character. Young (1990) states, "violence is systematic because it is directed at members of a group simply because they are members of that group" (p. 62). Women, people of color, and LGBTQ individuals live in fear of violence perpetrated upon them simply because they are members of an oppressed group. Young argues acts of violence against these groups are not used to simply keep control (as in repressive state violence) instead, the violent acts occur because of fear or hatred of the group and insecurities of the perpetrator. Given this irrational perception Young suggests these acts of violence are rooted in unconscious processes, particularly the fear of identity loss.

The Psychological Effects of Oppression. Through the five faces of oppression identified by Young, oppression takes a serious psychological toll. Biko (1978) goes so far as to argue, "The most potent weapon of the oppressor is the mind of the oppressed" (as cited in Watts, Griffith, \& Abdul-Adil, 1999, p. 257). Using theory developed by Frantz Fanon, Sandra Lee Bartky (2004) argues for three categories of psychological oppression: stereotyping, cultural domination, and sexual objectification. Each of these modes of psychological oppression shares the common tendencies of splitting and mystification. Splitting refers to the tendency to make the oppressed feel they are being divided into their "true" and "false" selves, while mystification refers to "the systematic obscuring of both the reality and agencies of psychological oppression so that its intended effect, the depreciated self, is lived out as destiny, guilt, or neurosis" (p. 25).

Stereotyping. Bartky (2004) argues stereotyping is oppressive because it limits the ability for individuals to respect stereotyped others as whole human beings. When 
stereotyped individuals receive this feedback from others, it is only a small step to start believing these misconceptions about themselves. When an individual cannot access their full range of humanity, they cannot achieve self-actualization. The self may be fragmented or split into stereotypical beliefs and behaviors and more authentic versions of the self or to use Bartky's (2004) example "We [women] cannot be autonomous as men are thought to be autonomous, without in some sense ceasing to be a women" (p. 26).

According to Ann Cudd (2006), stereotypes are the primary means of internalized oppression. In the minds of oppressors, stereotypes function to justify the oppression of some groups by means of dehumanization. Stereotypes also may lead to a process of acceptance of oppression on the part of the oppressed. The internalization of stereotypes leads to consciousness dependency or false consciousness. Consciousness dependency is the process by which the oppressed internalize the consciousness of the oppressor (Freire, 2000). The consciousness of the oppressor includes justifications of oppression that work by painting the oppressed as morally corrupt and/or lazy.

Cultural domination. Culture plays a role in psychological oppression because its global nature frames the boundaries of knowledge. Cultural domination and oppression may appear natural because of their all-encompassing character. In other words, it is hard to begin to question something that is seen as natural and immutable, making cultural domination a particularly insidious form of psychological control. It is hard to take pride in a group identity, which is either invisible or constantly downgraded by dominant culture. Further, by being exclusively connected to dominant culture, individuals may 
feel more connected to the oppressor than others may in their social group (Bartky, 2004).

Sexual objectification. While sexual objectification can be a complex phenomenon, Bartky (2004) argues it becomes clearly oppressive when a person's sexuality is extended into every area of their existence. Like stereotyping, being constantly sexualized, is to deny a person their full range of humanity and is another way through which oppressors seek to control the oppressed. Sexual objectification differs from stereotyping in that it is enacted on the bodies of the oppressed in an attempt to indirectly control these bodies in a time when direct control (e.g., slavery) is generally no longer an option. This process is most commonly associated with the unique oppressive experience of women but has also been used to oppress many other groups including, Black and working class individuals. In sexual objectification, the fragmenting of self takes on a physical form, as women may only become her breast through the unwelcomed male gaze. Like the other forms of psychological oppression, sexual objectification can take on a purely psychological role as women learn to evaluate their worth based exclusively on various body parts.

Complexities of psychological oppression. The process through which oppression functions at the psychological level is full of contradiction and complexity. In a particularly insightful passage into the psychological processes of oppression Bartky (2004) argues:

It is itself psychologically oppressive both to believe and at the same time not to believe that one is inferior - in other words, to believe a contradiction. Lacking an analysis of the larger system of social relations, which produced it, one can only make sense of this contradiction in two ways. First, while accepting in some quite formal sense the proposition that "all men are created equal", I can believe, inconsistently, what my oppressors have always believed: 
that some types of persons are less equal than others. I may then live out my membership in my sex or race in shame; I am "only a woman" or "just a nigger." Or, somewhat more consistently, I may reject entirely the belief that my disadvantage is generic; but having still to account for it somehow, I may locate the cause squarely within myself, a bad destiny of an entirely private sort-- a character flaw, an "inferiority complex," or a neurosis (p. 32)

Both of these potential responses to oppression result in a low level of self-efficacy and a poor view of self. The oppressed individual is either personally defective or part of a flawed group. Additionally, the complexity of the process suggests that psychological interventions around increasing self-efficacy and/or empowerment are overly simplistic in their conception of the problem at hand. The issue is not simply a feeling of a lack of power, but rather, a split consciousness. This process is the "false consciousness" discussed by Marx and the "consciousness dependency" discussed by Freire. The result of such consciousness fragmentation is negativity directed towards self or similar others (i.e., horizontal violence).

Bartky (2004) culminates her theory of psychological oppression by returning to Marx's historic conceptualization of alienation. Although Marx speaks of alienation in terms of not owning the products of one's labor, Bartky suggests alienation of labor is intimately connected to psychic alienation arguing both involve splitting of human capacities and forbidding certain human activities. She argues that:

... alienation occurs in each case when activities which not only belong to the domain of the self but define, in large measure, the proper functioning of this self falls under the control of others... From this perspective, cultural domination would be the estrangement or alienation of production in the cultural sphere; while the subjective effects of stereotyping as well as the selfobjectification that regularly accompanies sexual objectification could be interpreted as alienation in the production of one's own person (Bartky, 2004, p. 34) 
The psychological, physical, social, and economic effects of oppression are all intimately intertwined. Bartky concludes by arguing that types of oppression, e.g. racism or sexism, will not disappear one by one, instead the entire system of oppression must be dismantled. I add to this that addressing the social and economic effects of oppression is a necessary step in ridding the world of oppression but it is only a part of the solution.

The psychological effects of oppression on the oppressed and oppressor must be addressed if real, long lasting change is to occur. The above scholars' arguments that the current expressions of oppression are primarily covert- relatively invisible to an untrained eye- in nature suggests that the traditional tactics of change, which primarily focus on the external effects of oppression, are not enough to create sustainable change. External efforts at social change must be accompanied by changes in cognitions and emotions. This line of thinking is far from novel. The women's movement of the 1960's and 1970's focused on consciousness-raising in addition to more concrete change efforts. Consciousness raising groups grew out of the women's movement slogan-- the personal is political. In these groups women's experiences and feelings were validated as not just personal and isolated but communal and shared experiences that are political in nature and open for reflection and critique (Boler, 1999). These groups were central not only to widening individual woman's consciousness but to the process of developing solidarity and collective anger among women. Other social movements have also expanded their change efforts to include overcoming the psychological aspects of oppression (e.g., gay pride) (see Armstrong, 2002). Psychologists have concerned themselves with the idea of increasing empowerment and self-efficacy to help people help themselves. Each of these 
efforts has brought up something important about overcoming oppression but each also has weaknesses.

\section{Critical Consciousness: Origins and Current Conceptualizations}

Many methods of countering oppression have been proposed, both within academia and within social movements. I have chosen to focus primarily on a phenomenon developed by Paulo Freire in the 1970's, critical consciousness. I chose this concept because it requires the simultaneous challenge of both internal and external types of oppression. Further, leaders within the field of Community Psychology have suggested that critical consciousness is an important area of investigation for the field (Montero, 2009; Watts et al., 2011). However, the literature in the field is relatively scant and, as is addressed below, riddled with holes and unanswered questions.

Additionally, the choice to concentrate on critical consciousness was made because of my interest in education. Critical consciousness, while a psychological phenomenon, has historically been associated with the field of education. This relative lack of attention within psychology has meant that the psychological experience and understanding of the phenomenon has been under theorized. Before I begin my historic overview of thinking around critical consciousness, I address two important predecessors to the idea.

Freire, Marxism, and Psychoanalysis. The influence of Karl Marx on Freire's original conceptualization of critical consciousness is undeniable. Freire's ideas regarding critical consciousness are intimately connected to Marx's conceptualization of class-consciousness. This is seen in that both concepts are not inherent in individuals but 
must be developed over time. Further, Freire's conceptualization of consciousness mirrored that of Marx's. Specifically, he saw consciousness as developed through reflection on an externally existing material reality, which includes other humans, so consciousness is highly social in nature. Au (2007), arguing for the influence of Marx on Freire, suggests:

... dialectical materialism provides a framework for analyzing objectively existing conditions in the world (i.e., various forms of oppression), for understanding that humans can become actively conscious of both the conditions themselves and their sources, and for changing these conditions through human (social) intervention and action. (para. 6)

In a dialectic conception of consciousness, discussed more fully in the next chapter, the process of humans interacting with each other and material reality creates the ongoing formation of consciousness ( $\mathrm{Au}, 2012)$. This ongoing interaction with the world means that individuals can transform their consciousness and the material world through engaging in praxis - specifically, critical praxis.

To engage in critical praxis individuals must first engage in what Freire called "consciousness of consciousness" (as cited in Au, 2012). Au (2012) states that thinking about thinking, "requires retrospection and introspection, looking backward and inward to consider how our experiences and outward social structures shape our consciousness" (p. 25). Nevertheless, this awareness is not enough, individuals must also be able to critically question forces of domination, which are often invisible, and be able to envision and attempt to institute more progressive forms of social relations. However, this conceptualization of consciousness does not account for unconscious forces. A strictly 
Marxist conception of reality misses the unconscious but very real and important contributors to human consciousness and behavior.

Yet, psychoanalytically oriented scholars influenced Freire, particularly the work of the French-Algerian psychoanalyst Franz Fanon. Through his research and clinical practice in colonial Africa, Fanon (2004) showed that the experience of oppression is not only material but also covert and psychological in nature. The physical and psychological violence these communities suffered at the hands of colonizers and slave traders created a high degree of internalized racism that inhibited their capacity to work toward liberation. Freire (2000) speaks of a similar phenomenon, fear of freedom, which occurs when the oppressed cannot see (or even imagine) an alternative to their current position. This fear also keeps groups from working towards social justice.

Cho and Lewis (2005) suggest the oppressed also fear psychic loss. Using psychoanalytic informed theorizing, Cho and Lewis argue that individuals (whether they be objects of banking education or capitalism) have investment in their position as an acted upon object rather than an engaged subject. To become a subject would involve rejection of a sense of self and identity, which is invested in the object position. Disavowal of the object position causes intense feelings of anxiety and uncertainty. In other words, internal forces resulting from oppression include passionate attachments. Cho and Lewis suggest, "oppression has an existence in the unconscious such that those forces who are oppressed form passionate attachments to the forms of power that oppresses them...passionate attachments are power's psychic life" (p. $313 \& 320$ ). Oppressed individuals may have vested energies and identity in their role as an object. 
To lose this object status would constitute a radical loss (Cho \& Lewis, 2005). While Freire combines the Marxist and Psychoanalytic traditions of consciousness, his psychoanalytic focus is often not acknowledged in the everyday practice of Critical Pedagogy. This results in a lack of consideration of the emotions involved in developing critical consciousness. I address this repression of emotion in the field of critical consciousness further in the theoretical framework for this study (see chapter three).

Paulo Freire: The introduction of critical consciousness. Paulo Freire's Pedagogy of the Oppressed (2000) is the originator of many of the ideas central to this dissertation, including critical consciousness and Critical Pedagogy. Freire lays out a process of critical consciousness acquisition through a method of engaged, dialogic education, later named Critical Pedagogy. Freire (2000) theorized that the process of oppression dehumanizes both the oppressed and the oppressor. The dehumanization of the oppressed involves a process of portraying them as less than human, whether through stereotyping, sexual objectification, and various types of abuse and/or violence. Such forms of "Othering" may be deployed to justify the unequal distribution of resources in the minds of the oppressors. The oppressed are likely to experience a false consciousness or consciousness dependency, which involves an understanding of self and the world, which is dependent on the false consciousness of the oppressors. Consciousness dependency combined with dehumanization leads to internalized oppression, which may take the form of fear of freedom, self-blame, and/or learned helplessness. Each of these can play a role in the perpetuation of oppression. Individuals that cannot imagine alternatives to oppression may blame themselves, feel they deserve their oppression, or 
feel there is nothing they can do to change their oppressive situation. Further, individuals affected by these processes are unlikely to initiate or participate in actions directed towards social change. Nevertheless, this form of consciousness is not the only option for oppressed groups.

According to Freire (2000), oppressed individuals can move along a continuum of consciousness, which I call socio-political-cultural consciousness. The first stage, magical consciousness, is characterized by learned helplessness and adaptation. Oppressed people may not see their oppression or believe they deserve their inferior position, both reactions result from false consciousness. In this stage, individuals do nothing to fight oppressive systems, which are still invisible or seen as justified. In the next stage, naïve consciousness, individuals become more aware of oppression but place blame on other oppressed individuals (i.e., do not engage in systematic reasoning). Those on to which the oppressive situation is blamed may suffer from acts of horizontal violence. In the next and final level, critical consciousness, individuals develop a more nuanced view of oppressive systems, build in-group identity and pride, and begin to challenge oppressive systems in individual and collective ways.

Originally developed under the Portuguese term conscientização, critical consciousness refers to an individual's capacity to critically interpret their world and engage in individual and collective actions to change that world. Critical consciousness is a process, which involves the dynamic interconnected nature of reality and consciousness, and allows individuals to understand themselves and their world in relation to broad systems and institutions of oppression, colonization, and/or social 
injustice. Freire (1993), the originator of the idea of critical consciousness, described the concept as:

The critically transitive consciousness is characterized by depth in the interpretation of problems; by the substitution of causal principles for magical explanations; by the testing of one's "findings" and by openness to revision; by the attempt to avoid distortion when perceiving problems and to avoid preconceived notions when analyzing them; by refusing to transfer responsibility; by rejecting passive positions; by soundness of argumentation; by the practice of dialogue rather than polemics; by receptivity to the new for reasons beyond mere novelty and by the good sense not to reject the old just because it is old-by accepting what is valid in both old and new (p. 18)

The words of Freire reveal the breadth of his principles and the potential difficulties in operationalizing the concept of critical consciousness. As the quote suggests, critical consciousness results in a decrease in consciousness dependency, self-blame, and learned helplessness. It involves not only understanding one's place in a broad framework of injustice but also beginning to engage in actions to change the system. According to Freire, critical consciousness incorporates a formation of knowledge that involves critical thinking, participation in knowledge production, and an integration of individual and the broader community's concerns. As will be discussed in more detail in later sections, this definition is not without problems. Despite his dialectic commitments, Freire falls into the trap of splitting the rational and the emotional into disparate incompatible forces. One of the main goals of this dissertation is amending this split.

Individuals learning to read the world as they read the word facilitate movement along the consciousness continuum. To overcome barriers and initiate progressive change Freire (2000) hypothesized the process that is now called Critical Pedagogy, which advances a participatory model of education aimed at increasing literacy and the 
capacity to question, to seek alternatives to the given social order, and to experience a sense of social agency. In this philosophy of education, the students' experience is used as the primary foundation for the curriculum. In addition, the nature of the discussion is a dialogue between student and educator where everyone is both learning and teaching. Originally conceived to teach adults to read in the poorest areas of Brazil, Critical Pedagogy has been used in a variety of global contexts. Because of the association between critical consciousness and Critical Pedagogy, it is important to incorporate some basic aspects of Critical Pedagogy into any project focused on critical consciousness development.

Although Freire's work represents the roots of the critical consciousness literature, many branches of research now exist in a variety of fields. I offer an overview of the empirical research and theory building around critical consciousness over the last 40 years while highlighting the gaps within which this dissertation fits.

Critical consciousness literature 1970-2012. While critical consciousness has been taken up by a number of fields, I concentrate my literature review in the psychological literature on the topic. This section follows a roughly chronological organization and builds up to the theoretical model of critical consciousness to be used in my dissertation, which is presented in the following chapter.

William Smith: The meaning of conscientização. An early attempt to work with Freire's $(1993 ;$ 2000) stages of critical consciousness was made by Smith (1976). In Smith's attempts to operationalize and measure conscientização he developed a coding scheme for Freire's three stages of consciousness: magical, naïve, and critical. Three 
areas naming, reflecting, and acting characterize each stage of development. Naming refers to a person's capacity to name social problems in which they are embedded. Reflecting refers to where an individual places blame for oppression as well as a person's capacity for analytic reasoning. Finally, acting refers to a persons' cognitive and behavioral response to oppression and capacity for change.

Appendix C shows Smith's coding scheme for conscientização in the form of a list that shows the three levels of consciousness and the thoughts and behaviors associated with each of the three areas of conscientização. Smith claims that this conceptualization does not adequately capture the fluid nature of conscientização and argues that despite the static nature of the figure, conscientização development is a fluid process with two transition stages, "playing host to the oppressor" and "fanaticized consciousness" (p. 45). Smith and colleagues' work to develop a coding scheme for conscientização utilized an impressive process of development and testing. In addition, Smith's conceptualization represents one of the more dynamic and detailed pictures of the developmental pathway of critical consciousness. However, the scheme was developed with a very specific population, primarily rural Ecuadorians, potentially limiting its utility for other groups. Additionally, the scheme does not address Freire's lack of attention to affective dimensions of critical consciousness development.

Ignacio Martín-Baró: Conscientization as the central task for psychologist.

Martín-Baró (1994) argues for the centrality of conscientization to the field of psychology because psychology should be concerned with moving individuals from alienated, false-consciousness, to a more critical view of the world that eschews self- 
blame and hegemonic values. He argues eloquently for three processes involved in conscientization. First, similarly to other critical consciousness scholars, he argues for the centrality of praxis to the process of development. Only through acting on and changing reality can consciousness begin to change. Second, Martín-Baró suggests that as the veil of hegemony is lifted people begin to see and grasp the roots of oppression. Through this process of decoding he argues, "This crumbles the consciousness that mythifies the situation as natural, and opens up the horizon to new possibilities for action" (p. 40). Third, the continued role of praxis allows individuals to continue to not only discover their selves, their reality and transform that reality, but also, allows the capacity to imagine a new reality. Martín-Baró makes clear that there cannot be critical consciousness without action because only through acting on the world can the world be understood. On the subject Martín-Baró states, "No knowledge can be true if it has not attached itself to the task of transforming reality, but the transformative process requires an involvement in the process of transforming human relationships" (p. 41). A psychology that focuses on conscientization maintains a concentration on the individual but no longer suffers from a simplistic splitting between the personal and the social, instead the psychological and social are in dialectic relations with one another.

\section{Elena Mustakova-Possardt: Critical consciousness through Piaget and Kohlberg.} Mustakova-Possardt (1998) defines critical consciousness as a psychological construct that bridges thought and action. She breaks down critical consciousness into two common psychological phenomena, structural and moral development. Moral development is envisaged in terms similar to Kohlberg's stages while structural 
development is defined using Piaget's stages.

Mustakova-Possardt (1998) collected interviews from 28 individuals from a wide variety of social locations in the United States and in Bulgaria. She found one of the primary motivations of critical consciousness development was "the motive to establish a sense of identity" (Mustakova-Possardt, 1998, p. 17). Social identity appeared to occur via two pathways - one where moral concerns preceded the formation of social identity, and another where moral concerns did not exist before socialization into group identity. In addition to identity, agency was identified as a characteristic of critical consciousness development.

Mustakova-Possardt (1998) argues individuals on the path to develop critical consciousness differ from other individuals in three primary ways, they:

1) question the set of social relations and the larger social environment in which they find themselves, 2) feel compelled to make active efforts to transform their relationships with those social conditions in congruence with their understanding, 3) seek an alternative vision of how things should be on grounds of explicit concerns with issues of justice and equity. (p.15)

Of the three levels of critical consciousness identified by Mustakova-Possardt each was found to correlate with a separate developmental pathway. For example, individuals that maintain pre-critical consciousness throughout adulthood are likely to have had high levels of moral motivation before the complete development of structural skills. That said Mustakova-Possardt does hypothesize that certain circumstances may stimulate the development of higher levels of moral reasoning, even late in life.

Maritza Montero: Critical consciousness as deidealization and problemitzation. Montero (2009) argues for the often-overlooked role of consciousness, particularly 
critical consciousness, in social transformation. She breaks critical consciousness into two component parts, problemitzation and deidealization. Both of which are identified as simultaneously emotional and cognitive processes. Montero points to Freire's actionreflection praxis cycle as a method for facilitating the development of critical consciousness. The reflection component produces deidealization, which Montero argues is a necessary first step in building a more liberating consciousness. Deidealization allows an individual to separate out the naturalized ways of thinking that facilitate internalized oppression from more liberatory modes of thought. Once oppressive ways of thinking are made conscious, individuals can begin to problematize them and begin the process of imagining alternatives.

Following Freire's reasoning Montero (2009) argues for the origins of problemitzation in formal and informal educational settings, she argues:

... to problematize is to generate situations in which the people involved are forced to review their actions and opinions about daily events considered not only as ordinary circumstances, but also as inevitable because of their attributed essential way of being. This happens in such a way that their critical discussion leads them to being aware of oppression (p. 80)

Although Montero at times uses different language, the process is the same as that identified by Freire and other critical consciousness scholars. Through dialogue and praxis, the veil of hegemony is lifted and action for progressive change can take place (Martín-Baró, 1994). Montero reminds us that problemitzation will look distinctive in different settings and communities. In other words, the process must be tied directly to the community. While Montero acknowledges the emotional component of critical 
consciousness development, she does not offer a theoretically sophisticated mechanism for understanding its role.

\section{Elizabeth Carlson, Joan Engebretson, and Robert Chamberlain: Cognitive and}

emotional aspects of critical consciousness. Carlson, Engebretson, and Chamberlain (2006) used Freire's work to frame their photo-voice project in a low-income, AfricanAmerican community. In response to the findings of their project Carlson and colleagues state, "to our surprise, the project produced an unanticipated unleashing of emotional energy that resulted in new levels of individual and collective responsibility and participation" (p. 838). That the authors were surprised by the emotional component of the process speaks to the gap in understanding around affect and critical consciousness.

Carlson identified the levels of cognitive-emotional interpretations as passive adaptation, emotional engagement, cognitive awakening, and intention to act. According to Carlson and colleagues (2006), an individual in the state of passive adaptation presented narratives rooted in despair and anger. They suggest that these individuals presented as helpless and highly emotional. The next level, emotional engagement, involves narratives that are still rooted in despair and anger but also include the ability to question the status quo. Individuals in this stage are likely to ask, who is responsible in the face of injustice. The third level, cognitive awakening, involves an increased awareness of personal responsibility and an overall feeling of sadness. Finally, the most advanced level involves being able to envision alternatives to the current oppressive reality. At this level, the primary emotion is hopefulness because the person sees himself or herself as part of the solution. 
Carlson and colleague's (2006) study expands the world of critical consciousness to include emotions. However, their interpretation of the emotional aspect of critical consciousness development is overly dismissive and problematic. My first area of concerns centers around their overarching emphasis on taking on personal responsibility for change. While it is true individuals must feel they can be part of a change, Carlson and colleagues seem to overemphasize this without attending to the fact that change can be difficult because of all of the socio-political barriers that can be identified by the critically consciousness person. Without this added acknowledgment of very real barriers to change, the ethic of personal responsibility can quickly become conservative and result in victim blaming.

An additional issue with Carlson, Engebretson, and Chamberlain (2006) is they do not seem to have considered any literature on the role of emotions in social movements. This literature notes that anger is at times a very helpful emotion (they view anger as entirely regressive) and that hopefulness can be useful but it is often not an emotion that can be singularly sustained in social justice activism. Further, Carlson and colleagues make the classic binary distinction between reason and emotion. Their language strongly suggests lower levels of critical consciousness as characterized by being overly emotive and that higher levels are more reason oriented. In my theoretical framework, I suggest a more nuanced role of emotions throughout the process of critical consciousness development.

Matthew Diemer and colleagues: Critical consciousness and career

development. Matthew Diemer and colleagues have pioneered the role critical 
consciousness plays in career development in adolescence (e.g., Diemer \& Blustein, 2007). In general, higher levels of critical consciousness are associated with a better career development trajectory. Diemer and Li (2011) break critical consciousness into two component parts, critical reflection and critical action. Critical action is further broken into sociopolitical control or political efficacy and actual participation in social action. Looking for the contextual antecedents of critical consciousness Diemer and $\mathrm{Li}$ found "parental and peer sociopolitical support predicts sociopolitical control and social action, which in turn predicts voting behavior" (p. 1815). Counter to much theorizing on critical consciousness development, the authors found that students' perception of teacher support did not increase sociopolitical control. However, the authors' do suggest that this may be because the teachers were not utilizing Critical Pedagogy. Although a valuable contribution to the understanding of the development and benefits of critical consciousness, the exclusive focus on voting as an outcome is problematic in that it represents only one version of social action. A broader understanding of social action might show higher levels of social action on the part of involved youth.

\section{Roderick Watts: Critical Consciousness, Community Psychology, and}

Sociopolitical Development. Within Community Psychology, Watts's and colleagues work on sociopolitical development and critical consciousness has been central to the concept's integration into the field. In this section, I chronologically review Watts's ongoing theorizing and use of these concepts. Throughout the articles reviewed, Watts argues that he is developing critical consciousness and critical thinking to facilitate the broader area of sociopolitical development, which he maintains "helps oppressed people 
to recognize unjust social processes and acquire skills for social change" (Watts \& Abdul-Adil, 1998, p. 64). The series of articles presented in this section reviews the theory and empirical support for Watts and colleagues model of sociopolitical development and critical consciousness.

Young warriors and the five-stage model of sociopolitical development. Watts and Abdul-Adil (1998) present a "theory of oppression and sociopolitical development based on the work of Serrano-Garcia, Freire, and others" (p. 63). The authors also present data to support the development of critical consciousness in their Young Warriors program, which uses Freirean liberation education processes to work with young AfricanAmerican men. The cultural themes used include primarily hip-hop videos, which are critically analyzed to develop critical consciousness, a more complex vision of manhood, create cultural awareness, and facilitate social change.

Watts and Abdul-Adil (1998) presents a five-stage model of sociopolitical development. The full description of each stage is presented in appendix D. The least developed state is the acritical stage, which is characterized by belief in a "just world", internalized oppression, and feelings of powerlessness. The next level, the adaptive stage, is characterized by an acknowledgment of injustice but also "predatory, anti-social, or accommodation strategies...to maintain a positive sense of self and to acquire social and material rewards" (p. 67-68). In the middle level, the pre-critical stage, individuals begin to develop a critical awareness of systems of injustice and question adaptive strategies for dealing with inequity. The critical stage involves the rapid development of critical consciousness and increased motivation for social change. Finally, the liberation 
stage involves the salient "experience and awareness of oppression" (p. 68) as well as "involvement in social action and community development" (p. 68) and "critical consciousness is an established component of self" (p. 68).

To measure gains in critical consciousness Watts and Abdul-Adil (1998) link it to the concept of critical thinking, arguing that critical consciousness is the "sociopolitical version of critical thinking" (p. 69). Drawing on African-American culture and community specifically, the authors separate critical consciousness into the following categories: see and mean, inference, defense, judgment, and feeling, change orientation, action-system changes, and action-personal. To judge the change in critical consciousness over time, only youth's responses that could be labeled in one of the above categories were considered. Using the percent of responses labeled as critical consciousness, the authors argue that the level of critical consciousness did increase over the eight-session Young Warriors program.

In Watts's subsequent work on the Young Warriors program, he concentrates more on the concept of sociopolitical development. Watts, Griffith, and Abdul-Adil (1999) further develop the five-stage model of sociopolitical development (SDP), again drawing on data obtained from the Young Warriors program. Here the authors define SPD as "the psychological processes that leads to and supports social and political action" (p. 256). Watts and colleagues argue that sociopolitical development occurs when a person is able to comprehend multiple levels of systematic power relationships and their connection to social identities. Individuals must be able to understand that they may be both oppressed and oppressor in the same context and that an oppressed identity 
in one setting may be privileged in another. For example, a Black man must deal with the oppression they face as an African-American but also attend to the privilege they are granted by their gender. The authors also argue critical thinking and empowerment are prerequisites for sociopolitical development.

A deficit of the Watts and Abdul-Adil (1998) and Watts, Griffith, and Abdul-Adil (1999) work is that the data used to support the supposed increases in SPD are rather weak. Although it appears students did make strides in their ability to analyze the material, the responses that are labeled as critical consciousness are not convincing. The quotes offered in each text do not seem to actually illustrate a critically conscious way of thinking as they have defined the concept. For example, Watts, Griffith, and Abdul-Adil use the following passage to exemplify the development of critical consciousness:

Trainer: If you could change some things going on in this community, what would be some of the things you'd want to change?

Student: Have more security around.

Trainer: Have more security, keep people safe. What else would you change?

Student: People have to be in at a certain time.

Trainer: So the young folks get the type of guidance and learn [about] what they can do.

Student: You know what, around my house, [they say] have your boy out of here at $11: 30$.

[Trainers asking students for clarification].

Student: I think it would be a lot more Black unity, then all of this would be settled. You know, all the drugs and everything.

Trainers: Folks sticking together more?

Student: That don't make any sense, you know, everyone killing each other. As much as our race has come up, we steady breaking it down (p. 268)

Although the students in this interaction are producing action statements focused around changing communities it is important to note that the interaction starts with a prompt for such a statement. Further, the strategies the youth come up with do not seem to be rooted 
in a systematic or critical reflection on the world. Increasing security and enforcing a curfew do not seem to represent a high level of critical consciousness; rather these could be viewed as quite regressive response to the problem. This is not to take away from the impressive increases in development youth seem to show through the Young Warriors program but to suggest development is far from complete and could be characterized as a moderate level of SPD. A program that involved a higher level of time commitment might be better able to facilitate greater levels of SPD. Further, older individuals might be more cognitively developed and thus better able to respond to programs focused around increasing critical consciousness and SPD (Mustakova-Possardt, 1998).

\section{Critical Consciousness: Political Efficacy, Critical Action, and Critical}

Reflection. Watts, Diemer, and Voight (2011) review critical consciousness literature and suggest the concept consists of three parts: 1) "critical social analysis", 2) "political efficacy", and 3) "participation in civic or political action" (p. 45) arguing these aspects of critical consciousness are dynamically interrelated. Working with Freire, the authors argue for a "cyclical process of CC [critical consciousness] development" (p. 47).

Critical social analysis or critical reflection is the aspect of critical consciousness that most clearly separates it from other related concepts (e.g., civic engagement). Individuals displaying critical reflection move away from the tendency to victim blame and believe in the just world fallacy. Instead of blaming individuals for their problems, a more systematic understanding of injustice develops. Individual and group experiences are placed in a historical context of systematic oppression. Watts, Diemer, and Voight (2011) argue the reverse scaling of a Social Dominance Orientation Scale can quantify 
systematic understanding of oppression. Social dominance orientation is a classical social psychological concept that refers to individuals who prefer hierarchy in social relations because of their belief that people higher in the hierarchy earned the position. Watts and colleagues argue an inverse score on such a measure could indicate the level of critical reflection.

Political efficacy differs from the traditional psychological concept of efficacy in that it is directly related to an individual's perceived capacity to affect political or social change as an individual or part of a group. Two subcategories of the concept are external political efficacy -- "beliefs that government structures and officials are responsive to one's political interests" (p. 50) and internal political efficacy -- "people's beliefs about their capacity to be effective political actors" (p. 50).

Critical action refers to actual acts an individual or group takes to create change. Acts can range from traditional political processes, such as voting, to more justice oriented acts, to individualized expressions of resistance. To quantify critical action the authors suggest the use of the Activism Orientation Scale, which measures "how likely people will engage in political action taken to reduce perceived inequities" (p. 51).

Watts, Diemer, and Voight's (2011) review of the critical consciousness literature offers innovative suggestions around the quantitative measurement of the three theorized aspects of critical consciousness. However, the authors make no move to address the lack of nuanced understanding of the emotions involved in critical consciousness. I will take up this area of theorizing in my theoretical framework. Further, while Watts and colleagues' identification of survey measures that might correspond to different aspects 
of critical consciousness development is useful in the operationalization and measurement of the phenomenon, the step seems potentially premature. I say this because the model proposed by Watts and colleagues has had only limited exploration using qualitative methods, meaning that while it seems clear that the suggested quantitative measures have utility, they may be limited to the understanding of the full expression of the various aspects of critical consciousness development. In summation and critique of the existing literature on critical consciousness development in the next section, I offer a framework of critical consciousness development that incorporates affective dimensions of the phenomenon. 


\section{Chapter Three: Theoretical Framework}

In this section, I start by laying out my understanding of consciousness broadly, followed by my conceptualization of critical consciousness as the highest level of sociopolitical-cultural (SPC) consciousness development. I then move to address the external and internal dynamics of SPC consciousness. I bring a psychological lens to the model while integrating scholarship from a range of disciplines. This interdisciplinary focus centers specifically on building a theoretical rationale for studying the role of emotions and related concept of relationality in critical consciousness development.

The dissertation draws on a model that distinguishes three states of SPC consciousness, and frames critical consciousness as both a state and a process. While I am primarily interested in SPC consciousness as a process, having a picture of the states involved aids in my interpretation of the process-oriented data. I propose three states of development and identify the cognitive, emotional, and behavioral components that are theorized as characteristic of each state. I present these three states as ideal types, meaning that no actual case is likely to fit perfectly within any one state. I present the states as an anchor in understanding the role of the capstone course in the participants' ongoing process of critical consciousness development. The theoretical framework provided a guide for the development of the methods and analysis by sharpening the lens through which I viewed the data. That said, the qualitative nature of the study allowed for the emergence of other unforeseen themes and narratives, such as the expanded emphasis on relationality. 


\section{Dialectic Consciousness}

To study critical consciousness requires an understanding of consciousness more broadly. I root my understanding of consciousness in the idea of praxis as understood by scholars such as Hegel, Marx, Freire, and Fromm. Consciousness does not develop in an isolated individual psyche. Instead, consciousness involves internal forces (e.g., drives, cognitions, and emotions), which are dynamically related to the external world (e.g., material reality, culture, and ideology) in an ongoing process of the world acting on consciousness and consciousness (through behaviors) acting on the world (i.e., praxis).

The term dialectical refers to a mode of analysis that emphasizes tensions or conflicts between or among the various levels of the analysis. The dialectical approach looks at reciprocal relationships. Through a dialectic lens, we conceptualize the world as involving conflicting forces that are continuously changing (Marx \& Engels, 1968). Material reality effects consciousness and can be changed by consciously directed actions. Many action researchers, through the idea of praxis, conceptualize the dialectical relationship between consciousness and the material world (see Brydon-Miller, 2001; Martín-Baró, 1994). Praxis is the process of acting on reality, reflecting on action, and then acting again in light of those reflections. Praxis is a key driving force of SPC consciousness development. As emphasized by Martín-Baró (1994), the on-going process of thinking and acting on the world is a primary mechanism through which the false consciousness of hegemony is transformed to critical consciousness and associated critical action. Because the psychological and social are one, the reflection component of critical consciousness cannot be separated from the action component. This is also a 
primary reason why courses focused on increasing SPC consciousness need a direct action component of some nature but this direct action component must be tied to reflection.

I am concerned with a specific subset of dialectical consciousness, SPC consciousness, which looks at the level of awareness of social, political, historical, and cultural forces, which are often hidden from conscious awareness. This awareness brings to light previously invisible and normalized aspects of ideology that maintain oppression (Moane, 2010). Prilleltensky and Gonick (1996) argue that as the psychological mechanisms of oppression are uncovered and overcome the ability to perceive external sources of oppression (political, social, and cultural) results. However, they do note that awareness of the various levels of sources of oppression may differ. In other words, someone may have a high level of awareness of the dynamics of oppression in social groups but less awareness of mechanisms of oppression at state, national, and international levels. I expect that this awareness is tied to the type of oppression experienced and/or studied. For example, studying the oppression of women often involves an investigation into oppressive dynamics in the family but may focus less on the oppression of international forces (e.g., the constraints of global capitalism). Alternatively, the study of the oppression of the lower class may include an investigation into the role of transnational corporations and capitalism in perpetuating classism but may not attend to how classism plays out on a small group level. Ideally as a person moves along the SPC consciousness continuum towards critical consciousness they develop an increasingly complex understanding of oppression operating at multiple levels 
coupled with increasingly critical action on the world that addresses the multiple levels of oppression.

As stated above, the basis of my understanding of consciousness is rooted in Marx (and taken up by Freire), who understood that consciousness depends on an individual's relation to power. Those with power are unable (on their own) to see oppressive structures as they exist because they are essentially blinded by their position of power and dehumanized by the act of oppressing others. It is easier for those with less power to recognize the external structures of oppression. Feminist Standpoint Theory similarly argues the standpoint of those not in a position of power is inherently a more correct interpretation of the world (Hartsock, 1983). While I agree with these interpretations I argue it is important to remember internal dynamics can fog interpretations for privileged and oppressed individuals alike, hence the need for SPC consciousness in dominated and dominator groups. For marginalized groups, SPC consciousness development is a process of defogging their standpoint. For dominator groups the process involves learning from marginalized groups that have the power to transform themselves and those in power by creating an alternate reality not based in subject-object oppressive relations. Subject-object relations exist when the individual(s) with more power objectify those with less, dehumanizing them through the processes of oppression discussed in the literature review. One of the primary results of the development of SPC consciousness is the ability to engage in subject-subject positioning in which both parties are seen as fully human and possess the capacity to drive their own life. 
While helpful, the oppressor-oppressed distinction breaks down when one considers the intersection of multiple social identities as is now the dominant position in Women's Studies, Cultural Studies, and Critical Theory (see Collins, 1990; Crenshaw, 1991). Very few people conform to this prototype of the absolute dominator or are completely marginalized. Instead, individuals have a multitude of identities that place them at times in the dominator and at times in a marginalized role. Taking the position of intersectionality involves rejecting stable categories and the essentialist assumptions associated with these categories. Intersectional theorists also argue that oppressions are not additive, but rather, each specific intersection of identities is qualitatively different. For example, Black women do not merely experience the oppression of women and the oppression of Blacks in a straightforward additive way but rather experience a distinctive form of oppression. This is an expansion of Freire's theory as he held on to the simplistic oppressor-oppressed distinction throughout Pedagogy of the Oppressed. By

understanding the intersecting nature of oppression, individuals are more likely to obtain knowledge of the different levels of oppression and take action to alleviate oppressive forces through many different avenues of critical action.

\section{Feminist Developmental Perspective}

The work of Carol Gilligan provides a theoretical and methodological touchstone for the dissertation. Her theory of women's development offers a unique understanding of how women and girls' experiences, particularly concerning moral development, differ from those of men and boys. Gilligan (1982) argues that women and girls develop a relational dynamic that underscores their development and that this is undervalued by 
traditional development theorists that exclusively studied men and boys (e.g., Piaget, Erickson, and Kohlberg). Kohlberg's theory of moral development centered around six stages of development, with high levels of moral reasoning rooted in a sense of justice. On the other hand, Gilligan's theory also consisted of six levels of moral development but instead of being focused on justice Gilligan conceptualized or morality as involving an ethic of care.

My project offers a similar feminist critique of Freire and much of the subsequent work on critical consciousness. In others words, the masculine notions of rationality and individuality have shaped previous understandings of critical consciousness. A primary goal of my dissertation is to attend to the role of relationships and emotions in the process of consciousness development. This critique expands the understanding of the concept through the lens of women's experiences but also offers a more complex picture in going beyond models based exclusively on cognitive and individual processes. The theories laid out below suggest the centrality of solidarity relationships and emotions in moving along the SPC consciousness curriculum. Riger (1993) offers a useful summation of Gilligan's work and its relationship to mainstream psychology:

Gilligan (1982) contrasted this view of human nature with an alternate vision that emphasized relatedness and interdependence as central values of human experience... [mainstream] psychology takes as its highest value the emphasis on autonomy and separation over relationality (p. 285).

The moral capacities identified by both Gilligan (1982) and Kohlberg (2008) may work in combination to help facilitate the development of socio-political-cultural consciousness more broadly. Although the capacities identified by Kohlberg may be important in understanding the phenomena identified in this dissertation, Gilligan's 
theory provides a better fit in its focus on emotions and relationships as moral capacities that have been understudied and under-theorized.

A second aspect of Gilligan's work taken up in this dissertation centers on her developmental perspective. Critical consciousness is best understood as a process; individuals may continuously move forward or backward on the continuum of SPC consciousness. Further, once a person has achieved a high level of SPC consciousness there is no guarantee that they will stay at that level. In fact, maintaining critical consciousness requires substantial cognitive and emotional work. I propose a three level model of development. My model mirrors Gilligan's in that relationships are a driving force for moral development. Her self-in-relation-to-other model is organized around the relational capacities and the development of an ethic of care. Where Kohlberg identified modes of thinking that characterized levels of moral development, Gilligan introduced a relational model centered on a widening range of voices or subject positions that could be held in mind. However, my model of development is more dynamic than Gilligan's. Although I do present threes levels of development at the end of this chapter, I argue that movement may occur both forward and backward on a continuum of consciousness. I also view my phenomenon of interest primarily as a process, with the states concept introduced to aid in organizing the theory and understanding how individuals move between levels. Further, each level includes emotional, cognitive, and behavioral components. Individuals are not apt to exemplify any one category by fitting the characteristics in all three-component parts. For example, a person may have cognitive capacities that are in the realm of critical consciousness but emotional and behavioral 
capacities that are in the transformational state. Now that I have laid out the general model of development informing the study, the following section addresses some of the forces driving socio-political-cultural (SPC) consciousness development.

\section{Socio-Political-Cultural Consciousness: Driving Forces for Development}

The external factors thought to influence critical consciousness development include multiple levels, which all undoubtedly play an important role in various aspects of SPC consciousness. Community and developmental psychologists have argued for the importance of family, peer, and community support (e.g., relationality) in developing critical consciousness. Diemer and colleagues (2006) found that perceived external support for challenging racism, sexism, and social injustice from peers, family, and community was significantly related to increases in critical reflection but not critical action. The authors argue this disconnect could be due to: 1) "the real-world constraints that adolescence has upon the capacity for action" (p. 454), 2) lack of guidance in effective critical action, and 3) schools potentially playing a larger role (both positive and negative) than support from peers, parents, or community.

In a more recent study, Diemer and Li (2011) expanded their definition of support to include education. They found education did not play a significant role in the development of critical consciousness hypothesizing the lack of relationship between the factors may have resulted from not distinguishing among different types of classrooms. Traditional classroom environments have been shown to support the status quo (e.g., Freire, 2000; hooks, 1994). Conversely, classrooms that utilize Critical Pedagogy and 
service-learning do seem to increase levels of SPC consciousness (Landreman et al., 2007; Reason, et al., 2005).

Reason and colleagues (2005) suggest that college is one of the major avenues through which racial justice allies develop. Racial justice allies, defined as white students that work against racism, are potential models of critical consciousness. Precollege experiences and attitudes, such as white privilege, racial justice, minority experiences, and parental influence, increase the potential positive effects of college on critical consciousness development. However, even without these precursors, college, partially through its capacity to advance cognitive complexity, can increase the critical reflection and critical action components of critical consciousness. Reason and colleagues argue that course work related to race alone or when combined with diverse friendships can lead to a "sense of whiteness ${ }^{1}$." In fact racial justice oriented coursework was one of the strongest predictors of development into an ally for racial justice. Other factors related to racial justice action included, invitation and opportunity as well as support and white racial justice role models. While this model offers support to my argument that college experiences have the capacity to develop critical consciousness, it does not offer a way of understanding the internal dynamics of the process, a key focus of this psychological oriented dissertation.

In a recent review of the critical consciousness literature Watts, Diemer, and Voight (2011) argue for a three-component model of critical consciousness. They define

\footnotetext{
${ }^{1}$ A sense of whiteness if defined as moving past understanding race as "just the color of skin" to understanding the current and historic power dynamics involved in racial relations combined with high racial justice attitudes (Reason, et al., 2005).
} 
the first component, critical reflection, as "social analysis and moral rejection of social inequities...that constrain well-bring and human agency...view social problems and inequalities in systematic terms" (p. 46). Second, the authors suggest the role of political efficacy, which they define as "the perceived capacity to effect social and political change by individual and/or collective activism" (p. 46). Finally, Watts and colleagues argue for critical action, defined as "individual or collective action taken to change aspects of society, such as institutional policies and practices, which are perceived to be unjust" (p. 46).

I agree with Watts, Diemer, and Voight's (2011) assessment that these are the three core components of critical consciousness currently identified in the literature on the issue. However, this does not mean that these aspects adequately capture the breadth of the phenomenon. One of the goals of this dissertation is attending to the emotional and relational forces at play in SPC consciousness development. My model integrates emotion and relations as central to the reflective, efficacy, and action components of critical consciousness.

Two areas of internal dynamics theorized here concern cognitive and emotional factors. These factors are not mutually exclusive; rather they are dynamically related forces. While considerable literature has amassed on the role of cognitions in critical consciousness development, the role of emotions in this same process is less well understood. It is important to note that at times I separate the emotional, cognitive, and behavioral aspects of SPC consciousness for the sake of description and analysis. 
Despite this, I acknowledge the interdependence of these concepts in that emotions and cognition depend on one another and the edges between these concepts are blurry.

The lack of attention to emotions within critical consciousness literature likely stems from Freire's ambiguity on the subject, Sherman contends:

On the one hand, Freire states that we need certain emotions (e.g., love, mutual trust) in order for dialogue, and thus for education for critical consciousness, to develop...certain emotions are portrayed as essential to the critical, rational process of education... On the other hand, Freire talks about the necessity of overcoming emotionality which he sees as one of the prime characteristics of a naive and irrational consciousness. (Cited in Boler, 1997, p. 221).

This disconnect within Freire's theory is addressed more fully in other sections. Here it is important to note that this disconnect represents a substantial limitation of the theory that has affected the subsequent literature on the subject.

Megan Boler (1997) argues that emotions do play an important role in both rationalizing social control over groups defined as "too emotional" and in theorizing the emotional side of engaging in social movements. Coming from a feminist orientation, Boler suggests emotions have historically been used to exclude women from positions of power. The reason/emotion dichotomy has been used to argue that women's tendency to be over emotional makes them unfit for leadership positions. However, the women's movement of the 1960's began to see that emotions, such as anger, could be mobilized for political change. Since that, time feminist scholars have worked to break down the divide between reason and emotion, arguing that they are mutually connected and reinforcing. Feminist scholars have also contended that emotions are not private but socially constructed. For feminist and psychoanalytic scholars the realm of emotions and 
relationality are dynamically related. Many emotions are constructed not on an individual level but through group interactions.

The idea of the collective construction of emotion fits nicely with the transactional approach in this dissertation and helps explain how emotions develop not based just on internal phenomenon but also external forces such as, reactions of those outside the self and relationships with others. Shame is given as a prototypical example of this phenomenon, Bartky demonstrates:

...the 'feelings' and 'sensing' that go to make up the women's shame...do not reach a state of clarity we can dignify as belief. [Nonetheless] they are profoundly disclosive of women's 'being-in-the-world,' far more so than many of the fully formed beliefs women hold...such as...that they enjoy like men 'equality of opportunity' or that school and workplace is meritocratic in character. What gets grasped in the having of such feelings is nothing less than women's subordinate status in hierarchy of gender, their situation not in ideology but in the social formation as it is actually constituted. (Boler, 1997, p. 224)

The quote shows not only that shame is constructed collectively but also the limits of ideology in understanding shame. Boler (1997) also discusses the emotion of bitterness, arguing that no one individually feels bitter but is outwardly labeled as bitter by a dismissive other. In other words, bitterness results from the collectively constructed emotion of anger that is expressed but is not accepted by the intended recipient.

Feminists and psychoanalysts working within the relational tradition emphasis the importance of relationality and interdependence in the development of emotional and intellectual capacities (Poba, 2013). While this dissertation began by focusing on the emotional dimensions of critical consciousness development, the theoretical roots in feminism and psychoanalysis necessitate this be coupled with a focus on understanding relationships. This includes relationships between and among participants as well as the 
relationships between the participants and researcher. A key area of tension becomes understanding what Mitchell (1993) called "The most interesting feature of contemporary psychoanalytic perspectives on self is precisely the creative tension between the portrayal of the self as multiple and discontinuous and of self as integral and continuous" (as cited in Poba, 2013, p. 971).

Erika Summers-Effler (2002) offers a useful framework for understanding the emotions involved in developing critical consciousness. She starts by theorizing the difficulty involved in breaking from the status quo/dominant culture. Using women as an example, she discusses the dependency women feel towards men both concerning material resources but also emotional resources. In order for individuals to break the dependence on dominant culture, they must have a viable alternative for fulfilling these needs. Summers-Effler suggests that the emotional energy obtained through ritual interaction and, correspondingly, collective identity can fulfill these needs. Using Randall Collins (1990) work Summers-Effler argues that successful solidarity rituals involve "...face-to-face interaction, shared emotion, a shared focus of attention, and mutual awareness of this attention. This shifts the participants' awareness from themselves to the group" (p. 42). Ritual interaction provides a number of other benefits including “...the transient and immediately shared emotion, longer-term feelings of solidarity toward the group, longer-term individually oriented emotional energy, and an emotional-energy-loaded symbol of the solidarity interaction" (p. 42). These benefits combined with the shifting of awareness from self to group and society laid the groundwork for the development of critical consciousness. 
Summers-Effler (2002) then shifts to using the theory of Ariel Hochschild to further explicate the emotional-relational dimensions in shifting towards critical consciousness. Summers-Effler suggests that the emotional and cognitive energy involved in managing feelings, particularly deviant emotions, inhibit individuals' capacity to think critically about the status quo. However, through group ritual interaction emotions previously labeled by dominant society as deviant can begin to be normalized among group members a process, which further shifts blame away from the individual and towards society. This shift in blame creates anger that can now be consciously allowed because, as a previously understood deviant emotion, anger is now normalized through group interaction. Consciously experienced anger then opens the doors for frames of reference related to injustice to develop because cognitive and emotional energy are freed when anger becomes not only consciousness but normalized (i.e., no longer deviant). Injustice frames involve a cognitive reframing of previously held assumptions and worldviews through relational interactions.

Summers-Effler (2002) also argues that the critical action component of critical consciousness development requires much higher levels of emotional energy than the critical reflection component because the potential for harm is higher. She discusses this added challenge through the concept of hope, which is similar to Watts and colleagues conceptualization of critical efficacy. Summers-Effler describes how shame and fear are transformed into anger through high emotional energy. Further, even greater levels of emotional energy can transfer anger to hope, which is necessary for action. The emotional energy required for this second transformation can be facilitated through six 
avenues. First, is the ability to redirect positive emotional energy from a more privileged position to the fight for social change in another position. Second, structural level change, such as a slight increase in living standards can stimulate further action for change. Third, intense interaction rituals, such as women's consciousness raising groups or musical communities, can facilitate higher emotional energy. Fourth, a lack of reward from dominant culture can increase the capacity to bond and gain meaningful resources from the alternative group. Fifth, a charismatic leader can facilitate the process of increasing emotional energy by functioning as a symbol of the interaction ritual. Finally, intergenerational subculture can facilitate solidarity through widening the alternative community and providing role models. Overall, Summers-Effler's theorizing around critical consciousness development provides specificity into the emotional and relational dynamics involved in the process. However, even her micro sociological lens cannot fully account for the intrapsychic process of emotions and critical consciousness development, to attend to this dimension I turn to the psychoanalytically informed reasoning of Paul Hoggett.

Paul Hoggett (2009) offers a theory to attend more fully to the ambivalence involved in affective commitments to political struggle. Hoggett suggests that emotions are fluid and exist on the borders of consciousness and, as a result, what we feel often cannot be articulated. Currently within the critical consciousness literature the gap between critical reflection and critical action is beginning to be addressed, but only in regards to structure. In other words, people do not go on to act on their newly developed critical understanding of the world because the structures for them to act are absent or 
limited. While this is an important point to consider when theorizing the attitudebehavior gap, it does not adequately address the psychological processes that may be impeding action. Hoggett argues that a similar phenomenon has occurred in the literature on political mobilization. He suggests, "it was thought that the decision to mobilize was then based upon a calculation of the benefits to be derived versus the risks likely to be incurred by political action" (p. 80). This resource mobilization approach to understanding movement involvement does not adequately answer the questions of why people get involved in causes that do not directly benefit them or have a very small chance of success.

Hoggett (2009) goes a step further and argues that there is no "inevitable reason anger at injustice should lead to political action" (p. 80-81). He contends, similarly to Summers-Effler, that some factors, such as injustice frames and symbolic resources, may be mobilized to amend the reflection-action gap but that negative emotions, such as fear, shame, and despair, may disrupt this process. To overcome this disruption Hoggett suggests using shared emotions to increase agency and to overcome negative and limiting emotions. Summers-Effler's idea of emotional energy offers an area of expansion for Hoggett's theorizing in this area. As identified above, she suggests that through the normalizing of deviant emotions through group solidarity and ritual interaction, emotion can move from its limiting capacity in perpetuating reliance on the oppressive status quo to emotions empowering ability to increase the capacity for critical reflection and action. However, this form of thinking can be taken too far. It is important to also consider that shared emotions can be limiting. For example, early studies of social movements focused 
on the potential for regressive emotions to drive crowds of people to uncritical actions (see Blumer, 1951; Smelser, 1963). In theorizing the role of shared emotions in critical consciousness it is important to attend to its potential progressive and regressive functions.

In explicating the relationship between experiencing injustices and engaging in action, Hoggett argues, again similar to Summers-Effler (2002), for the importance of community and relationality. Alone an individual may blame experiences of injustice on personal fault, precluding an analysis of systematic problems. However, once in a community individuals can begin to see that their experience is not unique and represents a pattern of systematic and systemic injustice.

Hoggett (2009) identifies a psychological disjuncture often experienced between cognitions and emotions. This disjuncture may operate as another limiting factor in developing critical consciousness. Using climate change as an example, Hoggett contends that individuals cognitively understand the threat of global warming and know what to do to decrease the threat but emotionally individuals do not connect the threat to themselves. The development of critical consciousness is rife with opportunities for such dissonance. At any point in development, the cognitive, behavioral, and/or emotional components of the process may be in dissonance. Dissonance theorists suggest that experiencing such contradictions is uncomfortable and that we are motivated to resolve the incongruities (Festinger, Riecken, \& Schachter, 1956). Each experience of dissonance offers a person the opportunity to move forward or backward on the continuum of SPC consciousness development based on the process through which they 
alleviate the dissonance. For example, to overcome the dissonance of believing that social change is necessary and the behavior of not engaging in social change efforts a person may shift their behavior (e.g., begin to engage in change efforts) or change their attitude (e.g., decide that social change is impossible or unnecessary). The first option would result in moving towards critical consciousness while the later options would results in moving towards the opposite end of the continuum. Another likely area of dissonance in critical consciousness development would center on the conflict felt between dominant culture and the alternative group culture. Again, how individuals are able to solve such dilemmas will influence their SPC consciousness development. Hoggett (2009) argues for the importance of being able to complicate the picture and take the perspective of others, while still engaging in action. It is only through this form of action, which Hoggett calls reflexive activism, that movement can avoid replacing one oppressive group with another. This type of action maps closely on to the theory of praxis put forth by Freire (2000) and Martín-Baró (1994) and, thus, the type of action that ideally results from critical consciousness. Further, the ability to engage in reflexive action offers a corrective to the regressive capacities of shared emotions as stated above.

The emotional work necessary for successful action changes with the evolution of the acting organization. Hoggett (2009) suggests, "in the early phases, emotion work often focuses on overcoming fear, turning grief into anger, or restoring hope... during the decline phase, emotion work will often focus on handling despair and burnout" (p. 94). This view of emotion expands on the theory of Summer-Effler (2002), particularly in the 
dimension of managing ongoing critical consciousness. Burnout and despair are inevitable to any social change project so having a way of dealing with these negative emotions is essential to a theory of ongoing critical consciousness development.

Central to Hoggett's (2009) theory is the idea of the labile nature of emotions.

He states:

...sudden movement from one emotional state to another-from grief to grievance and anger and from shame to pride, respectively. The dictionary definition of labile reads thus: 'liable to displacement or change.' It strikes me that some, if not perhaps all, emotions have this labile character. But they are not infinitely labile. There is no necessary reason, for example, why grief should suddenly give way to jealousy or shame to spite. But there does seem to be good reason to believe that many emotions exist in an intimate and dynamic relation to others—grief/grievance, shame/pride, love/hate, despair/hope...I think that in some sort of way... it is fluidity that provides one of the conditions of human agency. (p. 94-95)

This dynamic theory of emotions helps to explain the emotional shifts necessary for critical consciousness development in Summer-Effler's theory. Hoggett argues that the labile nature of emotions is key to understanding their connection to agency. In other words, only through the ability of emotions to shift can limiting emotions, such as despair and hate, be transformed to empowering emotions, such as hope and love. Further, the labile nature of emotions helps explain how progressive groups can transmute positive emotions into regressive emotions, such as hate and despair. This labile nature of affective states also explains the tendency for critical consciousness to never fully be maintained. In other words, this dynamic prevents people from maintaining all aspects of critical consciousness at all times. It then becomes important to understand the internal and external influences that move a person towards more empowering or more limiting emotions. 
Involvement in social movement and social change efforts cannot be understood strictly from rational and cognitive perspectives, as the critical consciousness literature has attempted. Instead, scholars must begin to understand the varying emotions and relations that drive individuals to develop the capacity for critical reflection and engage in critical action.

Consciousness involves the process of acting and reflecting on the world. This process is affected by numerous external factors, which influence the types of action available, and reflection on those actions. Equally important are the numerous internal factors that the influence the actions engaged in and the understanding of those actions and the broader world (i.e., reflection). In summation, cognitions and experiences, such as exposure to diversity, justice oriented courses, and cognitive and moral complexity can prime a person to develop critical consciousness. However, dissonance and emotional energy drive the process of development. Emotional energy is produced through ritual interactions that also build solidarity and collective identity. Additionally, ritual interactions and relationality involves the justification of emotions previously believed to be deviant. The labile nature of emotions allows them to shift. Once these emotions are normalized, they no longer have to be managed, freeing up additional cognitive and emotional energy, which facilitates the ability to be self-reflexive. This process facilitates a blame shift from the individual to society. In other words, oppression is no longer seen because of personal deficit but rather as a societal ill. The shift in blame combined with higher levels of emotional energy shifts limiting emotions like fear and shame to more empowering emotions like anger, which is directed at the unjust system. 
The above summary deals mostly with the processes involved in developing the critical reflection component of critical consciousness. Like Watts and colleagues, SummersEffler argues for the importance of critical efficacy, although she ties this directly to emotions arguing specifically for the role of hope (this also supports MustakovaPossardt's argument). Summers-Effler suggests that anger can be turned to impotence if emotional energy is low. To facilitate the transformation from anger to hope emotional energy must be even greater, a process facilitated by six different means given above. Once hope is present, the most complex (emotionally and cognitively) aspect of critical consciousness can be achieved, engaging in critical action. Opportunity structures, social justice role models, and invitation to engage in action also facilitate engaging in critical action. Once obtained critical consciousness must be maintained, a process SummersEffler suggests is done through sustained involvement in critical action and interacting in a critical community, both of which work as a positive emotional energy feedback loop.

While the above points to areas of agreement between Hoggett (2009) and Summers-Effler (2002), I use one area of Hoggett's work to further draw on the processes suggested by Summers-Effler. While she argues almost exclusively for the role of more long-term, collective emotions or what she calls emotional energy, Hoggett offers a useful way to conceptualize transient or more short-term emotions and how their shifting labile nature, which results from cognitive complexity and dissonance, can also be a driver towards or away from critical consciousness. I now move to address these three parts at the three levels of SPC consciousness: pre-consciousness, transformational consciousness, and critical consciousness. 


\section{Three States of Socio-Political-Cultural Consciousness}

SPC consciousness is a dynamic phenomenon in which individuals may move forward or backward on the continuum of development. I discuss pre-consciousness, transitional consciousness, and critical consciousness as states or levels rather than stages. These terms suggest movement that is more fluid; at times individuals may move towards critical consciousness and at other times towards pre-consciousness. Further, individuals may be at different levels in diverse contexts and/or may exist between states or have aspects from the three components in different levels. In other words, individuals are not going to exist as a perfect example of any one state. Instead, the levels should be considered as ideal types, which are used to compare individuals and groups and aid in being able conceptualize the process of development.

A handful of classifications of critical consciousness development exist but I have chosen levels as named by Mustakova-Possardt (2004). These terms best capture what I expect to see at each state, particularly the range, turmoil, and dissonance associated with the middle level. Further, most scholars argue for a three level model of development (e.g., Freire, 2000; Smith, 1976,). I incorporate other scholarly work into theorizing around each state where appropriate. Like Pitner and Sakamoto (2005), I argue for three primary components of each level of SPC consciousness: cognitive, emotional, and behavioral. It is important to note each of these domains is dynamically interrelated, so a perfect separation among them is not possible. Although each name does not explicitly state what type of consciousness, it should be understood I am speaking only of SPC consciousness. Below I identify the characteristic of the three levels of SPC 
consciousness development. For each state, I start by discussing the cognitive aspects, as these are often the first to develop. I then move to talk about the emotional dynamics for that state as they seem to play a role in moderating between the development of reflection and engaging in subsequent action (Hoggett, 2009; Summers-Effler, 2002). That said, it is important to note that thoughts, emotions, and behaviors all exists in dynamic relation to one another. I end by discussing the behaviors associated with each level of development.

Pre-Consciousness. The first level of SPC consciousness development is preconsciousness. As the name suggests, in this status there is no SPC consciousness. Overall, consciousness of consciousness is low and the understanding of the effect of internal and external factors on consciousness and behavior is even lower. MustakovaPossardt (1998) and Hoggett's (2009) theories both suggest that cognitions and affect are highly disconnected at this level and cognitive abilities overall may be quite simplistic (Reasons et al., 2005).

Cognitive. At this level of SPC consciousness individual thoughts are primarily focused around issues of survival (e.g., food, water, shelter) (Freire, 1993; Smith, 1976). Watts, Griffith, and Abdul-Adil (1999) argue "resource symmetry is outside of awareness... asymmetry is thought to result from real differences in capabilities of group members" (p. 263). This quote shows the two alternative orientations to injustice at this level. First, is a denial of the existence of injustice in social structure; denial could result from lack of contact with others of different social statuses (see Reason et al., 2005) or an unconscious block on seeing and acknowledging injustice (Summers-Effler, 2002). A 
second interpretation of injustice is to acknowledge it but to blame the victims of injustice (even oneself) for their plight. This understanding may continue into early transitional consciousness.

Individuals who reflect on social problems often make simplistic causal relations and/or blame problems on a superior power (e.g., luck, god, fate) (Freire, 1993; Smith, 1976). Belief in the just world fallacy- the tendency to trust the world is fair and people get what they deserve- is strong in this level (Watts \& Flanagan, 2007). This belief has been associated with blaming the poor for their situation and justifying through ideologies, such as the American Dream, or through religious beliefs, like karma. Individuals do not see problems outside of biological necessity and only biological explanations can sway their thinking (Freire, 1993; Mustakova-Possardt, 2003). Finally, in the pre-consciousness state individuals are disconnected from history and historical influences and thinking is highly binary (Freire, 1993). In other words, there is little room for ambiguity or cognitive complexity (Reason et al., 2005). At this level, individuals are not aware of emotional and/or unconscious influences on behaviors and only mildly aware of the affect of cognitions on their behavior.

Emotion. At this level, emotions of empathy and dependence on the oppressor are common (Smith, 1976). Further, individuals may feel inherently inferior to those with more power (Freire, 2000). Other expected feelings at this status include: fatalism, resignation, apathy, and helplessness (Carlson et al., 2005; Smith, 1976). Boler, Summers-Effler, and Hogget theorize that inhibiting feelings, such as despair, hate, fear, grief, shame, will dominate in this level of SPC consciousness; although, the empirical 
support for these claims is limited. In this state moral emotions are aligned with dominant ideology (Jasper, 2011). This level is characterized by an overwhelming feeling that things cannot be changed or do not need to be changed.

Summers-Effler's (2002) model suggest that emotions play a role in the feedback loop that keeps people stuck at this level of SPC consciousness. In her model, individuals are driven by the need to attain increased emotional energy but at this state individual do not have access to alternative sources of emotional energy outside of the limiting dominate culture. Further, for oppressed groups the highly complex and energy extracting process of managing emotions labeled as deviant limits the ability to self reflect and develop cognitive complexity.

Behavior. Individuals at this level do not engage in critical action. Instead, they may engage in a few service-oriented behaviors but at a much lower rate than in the transitional level. In this state, the overarching concern of survival and belief in a just world decreases motivation to engage in caring or charity oriented behaviors. Adding to the tendency for inaction is overwhelming fatalism (Freire, 1993; Smith, 1976). Adaptive behaviors are prevalent at this state; Watts, Griffith, and Abdul-Adil (1999) discuss the role of adaptive behaviors as "predatory, antisocial, or accommodation strategies [which] are employed to maintain a positive sense of self and to acquire social and material rewards" (p. 263). In addition, individuals at this state may be highly disconnected from communities of similar social status (Hoggett, 2009). Developing solidarity to similarly positioned others is one of the driving forces for the second level of SPC consciousness. 
Transitional Consciousness. The transitional level of SPC consciousness encompasses the widest variety of thoughts, emotions, and behaviors. It is at this state individuals begin to transition out of pre-consciousness and towards critical consciousness. The transformation is a highly emotional process because it involves significant changes to previous ways of knowing and being (Boler, 1999). It is during this level that individuals shed previous ways of thinking, feeling, and acting and replace them with new strategies and worldviews (Summers-Effler, 2002).

Mustakova-Possardt (1998) argues, formal operational thinking is fully developed at this level, thus thinking can move past the immediate concrete situation and abstraction becomes possible. Individuals begin to see patterns in social reality and feel their connection to these patterns (Mustakova-Possardt, 1998). Similarly to Boler, MustakovaPossardt (1998) argues:

...we also see people with transitional critical consciousness deal with a lot of internal contradictions. The incomplete decentering accounts for frequent conflicts between prejudice and stereotypical thinking in some areas of social life, and the general striving toward establishing meaningful and wholesome relationships. (p. 22).

In addition, this state allows more connection between cognitions and emotions. The process of engaging in ritual interactions with similarly positioned others builds emotional energy, solidarity, and collective identity that function as a driving force in this level (Summers-Effler, 2002). In summation, this level is a time of great change and turmoil.

Cognitive. At this state, individuals begin to see social injustice but they also accept the explanations for those injustices of those in power (Freire, 2000; Smith, 1976). 
The result of accepting dominator ideology is "self and peer deprecation, blames ancestors, and self-pity" (Smith, 1976, p. 43). Although individuals begin to see the inequality around them, they are likely to blame themselves or others in their social group for the injustices. This causes a number of negative psychological consequences, such as low group pride, low self-efficacy, and cognitive dissonance. Cognitive dissonance occurs as individuals want to think positively of themselves and of their group but are receiving negative messages from society and are not able to reconcile this disconnect. Reason and colleagues (2005) argue that development in this state can be facilitated by exposure to diversity, increasing cognitive and moral complexity, and the enhanced ability for self-reflection.

Watts, Griffith, and Abdul-Adil (1999) suggest of this state, "Complacency gives way to awareness of and concerns about asymmetry and inequality... there is a desire to learn more about asymmetry, injustice, oppression, and liberation” (p. 263). Individuals not only acknowledge inequality at this level but may also move towards an understanding of injustice, although root causes are likely not yet identified. That said, Montero (2009) argues toward the end of this transitional level, doubts about hegemony and critical analysis of circumstances begin to develop.

Carlson, Engebretson, and Chamberlain (2006) argue that thinking about social issues starts to center around the question, who is responsible? As individuals move along the continuum towards critical consciousness, they may begin to understand personal responsibility in changing social structures (Wallerstein \& Sanchez-Merki, 1994). At this level of SPC consciousness, thinking becomes more complex and critical. However, 
thinking is still simplistic in that it generally splits groups into all good or all bad and the ability to take the perspective of others is limited (Hoggett, 2009). Additionally, in this state individuals become more aware of their thinking, particularly how external forces influence their actions and reflections. Through this process (and the emotional dynamics identified below), blame begins to shift from the individual to the outside (SummersEffler, 2002).

Mustakova-Possardt (1998) suggests of the transitional status, "the striving toward consistency, integrity, service, and compassion is strong enough to foster the gradual evolving of a social consciousness oriented toward positive social change" (p. 22). Individuals trying to resolve feelings of upheaval in this stage may move towards critical consciousness to relieve these tensions. However, Freire (2000) argues that this tension could be resolved in other, more regressive ways. He suggests individuals in the transitional state (he calls it naïve transitivity) could instead move to a level of fanatic consciousness where they staunchly accept only one side of any issue, whether it be the new or the old position, the left or right. In the one-sided acceptance of only one form of argument, individuals deny the possibility of any wrong in their orientation or any right in the others-- a position, which is problematic to progressive development. Another potential move to deal with the tension in this state could be to revert to the pre-conscious level.

According to Mustakova-Possardt (1998) a primary advance in transitional consciousness is "the birth of problematizing and increasingly dialogical relationships with social reality... The successful progressive shedding of contradictions, stereotypes, 
and prejudices" (p. 22). She continues, a primary limitation of transitional consciousness is "its lack of systemic understanding, the result of which is that multiple social patterns and pathways of activism may be experienced as overwhelming when one is committed to bringing about positive change" (p. 22). Therefore, in this state cognitions are becoming increasingly attuned to reality but thinking still lacks a systematic quality.

Emotional. Internal forces may begin to move into awareness in this level but during this transitional level they may play a stronger role. Guishard (2009) suggests that this level is marked as a time of fear and vulnerability. This is not surprising given the cognitive processes that are occurring (i.e., becoming more aware of oppression). Pitner and Sakamoto (2005) argue that as an individual comes to understand the world that sees them and their group as negative they still desire to see themselves and their group as positive. This dissonance results in feelings of fear, anger, anxiety, and hostility. Pitner and Sakamoto (2005) suggest “The more an individual's self-image is challenged, the more uncomfortable she or he will feel... challenges and threats motivate a person to want to modify his or her world view" (p. 688). Here we see the potential for the affective to drive the process of SPC consciousness development. However, Pitner and Sakamoto suggest an individual may also respond to these challenges by desperately clinging to old ways of thinking. In other words, the affective component may have a positive or negative effect on SPC consciousness development.

Another important affective process in the transitional status is what Freire (2000) and Fromm (1994) label the fear of freedom. According to Freire (2000), "freedom would require them to reject this image [dependence on the oppressor] and replace it with 
autonomy and responsibility. Freedom is acquired by conquest, not by gift" (p. 44). In other words, individuals are afraid of the struggle and change required to reach an alternative way of being and interacting with others. Alternatively, Cho and Lewis (2005) argue that this process could be more accurately labeled as fear of radical loss. Individuals are not afraid of freedom but rather of the initial feelings of loss required to cast aside traditional ways of thinking and feeling which make up the self.

As Freire recognized, overcoming fear is a major step in this transformational stage, a view shared by Jasper (2011) and Hogget (2009) who contend that it is not simply fear that must be overcome, but rather, transformed into an emotion that increases the ability to engage in critical action. Similarly, other limiting emotions, such as grief, shame, despair, and hate must be transformed into activating emotions such as, grievance, pride, love, and hope. This may take place for a number of reasons, including a moral shock, development of shared feelings in a community, or increased alignment between cognitions and emotions.

Summers-Effler (2002) suggests a theory for this process. She argues that limiting emotions like shame and fear can be transformed into more empowering emotions through emotional energy. Emotional energy can be obtained through engaging in intergroup relations that involved ritual interactions that build group solidarity, collective identity, and normalize emotions previously identified as deviant.

Boler (1999) argues the struggle to unbind the sense of self from dominator ideology involves a feeling of extreme change as previous ways of thinking and feeling are critically interrogated. This change may cause strong emotional reactions, which 
must be attended to if individuals are going to continue to develop towards critical consciousness. Further, alternative ideologies must replace the previous ways of thinking.

Behavior. Although individuals begin to see injustices, they also feel as though they cannot change them, and adaptive behaviors continue although they may begin to be questioned. Alternatively, individuals at this state may begin rejecting all behaviors associated with the dominant culture (i.e., rebel against dominant culture). This can become problematic when it includes dismissal of potentially beneficial behaviors, such as engagement in educational settings (see Valenzuela, 1999).

In this state, some individuals may begin to take action to help others. However, this action is rooted in caring or charity, not justice (Wallerstein \& Sanchez-Merki, 1994). Cipolle (2010) distinguishes among types of service by arguing charity is "it's mainly about me", caring is "it's all about them", and social justice "we need to work together" (p. 48). In the transitional level of SPC consciousness, engagement in social justice oriented behaviors is relatively rare. Additionally, individuals at this level may begin to join communities of similar others. The behavior of joining a community affects emotions as individuals may begin to develop shared emotions that promote engagement in action (Summers-Effler, 2002).

Critical Consciousness. The last status on the continuum is never fully attained, but instead may be conceived as an ongoing process of moving towards critical consciousness. This level is characterized by critical action, critical efficacy, and critical reflection (Watts et al., 2011). In this level, individuals feel strong overlap between 
thoughts, emotions, and behaviors because individual have successfully navigated the dissonance felt at previous states by moving through them progressively. As stated above, this dissonance can also result in individuals moving backward on the continuum of SPC consciousness. Mustakova-Possardt (2004) says of critical consciousness it "is a precarious balance between mind and heart, where each serves as a corrective to the other, as a result of which the faculties of love, knowledge, and will function in relative unity" (p. 260). This stands in contrast to the divisions and contradictions occurring in the previous levels. The increased overlap between cognition, emotions, and behavior means that the divisions presented in this section are better understood as three concentric circles that become increasingly overlapping as an individual moves through the levels of SPC consciousness.

Cognition. Critical reflection involves understanding how systems of oppression operate including the ability to see "system as cause" and "contradiction between rhetoric" in addition the ability to "generalizes from one oppressive system to another" (Smith, 1976, p. 43) while being sure not to over generalize and assume different types of oppression are overly similar. At this level, individuals also begin to become aware of these assumptions and how they shape their interpretation of reality (Freire, 2000). This involves uncovering external and internal drivers of thoughts, behaviors, and emotions. This process is part of what drives the consistency of these dimensions of critical consciousness. In short, critical reflection involves the unveiling of oppression. At this level the awareness of oppression should begin to address both the intersection of 
different forms of oppression (Moane, 2010) and the interworking of oppression at multiple levels (Prilleltensky \& Gonick, 1996).

At this level, individuals gain a historical understanding that connects them to both histories of oppression and histories of resistance to oppression (Freire, 2000; Watts, Williams, \& Jaggers, 2003). Individuals can only move from the transitional level to critical consciousness when they can imagine alternatives to the current unjust reality and area aware of the possibility of change (Moane, 2010). Further, individuals believe they can change the current reality into something more desirable (i.e., political efficacy) (Diemer \& Li, 2011). Watts, Diemer, and Voight (2011) argue political efficacy involves an internal component or belief in oneself to create social change and an external component that involves belief in structures that can be changed. These characteristics in part drive the critical action component of critical consciousness but are also intimately tied to the emotional dimension.

Critical consciousness also involves an enhanced level of critical thinking, including: truth seeking, open-mindedness, analytic skills, and mature judgments (Watts, Abdul-Adil, \& Terrance, 2002). Further, individuals in this status become increasingly comfortable with ambiguity, which keeps individuals away from fanatic consciousness. In other words, individuals that are able to deal with dissonance without turning to simpler ways of understanding and acting on the world (i.e., moving backwards on the continuum) are able to deal with the complexity of the inner and outer world in a way that allows for all aspects of critical consciousness to develop (Hogget, 2009). This process is also facilitated by emotional dynamics. For example, Summers-Effler (2002) 
argues that shame or fear can be transitioned to anger and then hope through high emotional energy. It is this hope that allows for engagement in critical action.

Alternatively, shame, fear, and/or anger can be transformed into a feeling of impotence, which does not allow for critical action, through low emotional energy.

A key striving of this state is what Gramsci called "pessimism of the intellect, optimism of the will." In other words, a person develops the ability to be a critical intellectual, meaning they are open minded but also only accepts information that is accompanied by a strong argument and persuasive evidence. The second part of the quote deals with the emotional dynamics of this level; as injustice frames are formed and a fuller picture of unjust reality is built a person must also have the "optimism of will" or ability to act and persevere in the face of almost seemingly certain failure.

Emotion. The affective dimension of the final state of SPC consciousness involves rejecting negative images and stereotypes and replacing them with pride in previously marginalized identities (Freire, 2000). Carlson, Engebretson, and Chamberlain (2006) argue, as individuals begin to realize their responsibility to act they initially feel sadness but then, as they learn to envision alternative futures, the primary emotions become hopefulness. Summers-Effler also suggests that hope is one of the most central emotions to critical consciousness development because it allows for engagement in critical action. Guishard (2009) addresses a tension in the affective dimensions of social change, stating "grievance, anger, and frustration are often discussed as key motivating emotions to engender activism" but in his study he found "the foundation of the... critical consciousness was insurgent love and concern" (p.101). 
Mustakova-Possardt (1998) makes similar arguments regarding the centrality of love to engaging in critical action. Evidence seems to support the role of love and other positive emotions in this final state of SPC consciousness, but I am not convinced this is the full story. Working to create change in often seemingly unchangeable systems is emotionally quite taxing and not exclusively positive. Further, love may just be serving as a proxy for solidarity and collective identity, two key components of this level.

Summers-Effler (2002) suggest that the emotions involved in engaging in critical action must be much stronger than those involved in developing critical reflection. This is because the potential negative consequences are higher for critical action than for critical reflection. She argues that the emotional energy gained through ritual interaction is not enough to engage in action (this process is laid out in previous sections).

Hoggett offers more theoretically specificity for how and why emotions may shift. Hoggett (2009) argues that efficacy and emotion are intimately connected. He contends that the shift between limiting emotions (e.g., shame, hate, despair) to empowering emotions (e.g., pride, love, hope) increases individual action to affect change. Although Boler (1999) argues that anger is a likely factor in motivating political action, Hogget argues that anger is not enough. Anger when combined with other motivators' leads to action. Jasper (2011) offers a process through which emotions might lead the way to engagement in critical action. Emotional liberation or the shift from limiting emotions to empowering emotions and the simultaneous rejection of the dominant paradigm is likely a key factor in the this last level of SPC consciousness. This process mirrors Summers- 
Effler argument that deviant emotions must be normalized and shared before one can fully develop into critical consciousness.

Behavior. The primary outcome desired through the development of SPC consciousness is involvement in critical action. Watts, Griffith, and Abdul-Adil (1999) argue adaptive behavior decreases during this level, as involvement in critical action becomes an established component of self. What exactly counts as critical action has been heavily debated. Some critical consciousness scholars have used voting as a proxy for critical action (see Diemer \& Li, 2011). However, critical action involves a much wider range of behaviors. Following the birth of identity politics the nature of critical action has changed. Development of positive and alternative identities and cultures has been an increasingly important part of social movements in the United States (e.g., Bernstein, 1997).

Critical action should also strive to create systematic and/or institutional change as well. Involvement of this nature might include creating alternative communal based settings and organizing protest and/or boycotts. Behaviors, such as lobbying and advocacy might also be considered a form of action. The role of traditional governmentbased change actions is a hotly debated issue within academia and activists circles. Evidence seems to suggest that some radical individuals, particularly youth, have lost faith in the formal governmental apparatus of change and thus no longer participate in them. Given this, it is important to expand the definition of critical action to include alternative systematic behaviors (e.g., protest) and interpersonal and cultural oriented behaviors (e.g., interrupting oppressive language). 
Watts and colleagues (2011) suggest that it is important to note the factors outside the individual impact whether a person does or does not engaging in critical action. Structural opportunity and invitation to engage in action are both important factors. Engaging in action is hard enough, without having to look desperately or even create opportunities for action. That said, someone has to go through the process of creating opportunities for action so this is an important point to consider when working with individuals that already possess a high level of critical consciousness. Further, the presence of role models and structures that promote action make engaging in critical action more likely (Reason et al., 2005).

Once the critical consciousness level of SPC consciousness is developed, maintenance of this level becomes important. Summers-Effler (2002) suggest that sustained involvement in critical action and a critical community are necessary to maintain the high levels of emotional energy necessary to maintain critical consciousness. Reflecting on these actions through praxis is also an important aspect of maintaining critical consciousness. Summers-Effler (2002) suggest the process of continued engagement functions as a positive feedback loop of emotional energy through the interaction rituals and collective identity involved in critical community and associated critical actions. Interestingly, the ability for activists to define almost any action as a "success" is important to this feedback loop. While social movement scholars have been arguing over what "counts as success" (see Gamson, 1990; Piven \& Cloward, 1979) it appears that for the sustained critical action, almost any outcome should be labeled as a success. So, while achieving social justice is the overarching goal, the outcome of 
building community and collective identity must be labeled as success to maintain member involvement. Moane (2010) suggest that ideally critical action is being taken at multiple levels. Because oppression exist at multiple levels effect action must address oppression at these different junctures.

Overall, critical consciousness involves a precarious balance between self and group, positive and negative, realism and optimism, and reflection, action, and behaviors. Critical consciousness can be understood through trying to comprehend the tensions involved in its development and maintenance. Having laid out my understanding of SPC consciousness development I now move to the design of this study. In the next chapter, I address the primary questions of the dissertation and the methods that I used to address these questions. 


\section{Chapter Four: Research Methods}

A central organizing principle of Community Psychology has been the call to understand people in context. Seminal works, such as Trickett (1996), call for the necessity of contextualized knowledge that attends to historical and environmental factors. In this model of studying psychology, individuals are seen as embedded in a particular society, culture, community, neighborhood, and/or family at a particular moment in time. This orientation to knowledge production counters that from the current dominate positivistic paradigm in psychology.

Psychology, for the most part, has been concerned with searching for universal human tendencies. The goal has been to remove the context as much as possible by utilizing experimental methods and statistical controls. This has provided the field with invaluable insight into individual psychologies but these highly controlled environments do not adequately reflect reality. The knowledge we gain from experiments is only one way of understanding reality.

Burton and Kagen (2005) argue that the general tendency within psychology to concentrate on experiments and hold onto belief in neutrality have created a socially irrelevant science that holds back a psychology that could be more focused on human liberation. Critiques such as Burton and Kagen, and Martín-Baró's (1994) similar position on the limitations of this paradigm, have been informed by Latin American Community and Liberation Psychologies. A parallel critique has sprung up in Community Psychology in the United States. Early articulators of this position, 
Rappaport, Trickett, and Kelly, called for an increase in studies that look at people in contexts and try to understand how the person and environment interact.

To get at the complexity inherent in context requires a new orientation to studying psychology. This new lens requires researchers to look closer and deeper. The quest for generalizability is replaced with a concentration on the small-scale and local. Qualitative approaches became the methods of choice for researchers concerned with contextualizing knowledge. Advocates for the use of qualitative methods in Community Psychology include, Prilleltensky and Nelson (1997), Stein and Mankowski (2006), and Trickett (1996).

In addition to the contextualization of knowledge qualitative methods, offer other advantages to community psychologists. Prilleltensky and Nelson (1997) argue that working towards social justice is a primary value for Community Psychology. Qualitative methods, particularly those that involve narratives, have the capacity to aid in the change process by facilitating the spread of previously marginalized voices (Rappaport, 1998). Memories and stories can be powerful forces for change in that they build identity, emotion, and memory and they cut across multiple levels linking the individual and the social (Rappaport, 1998). Another advantage of contextualized, qualitative methods is their ability to get away from explanations that blame the victim. Trickett (1996) explicitly states that the tendency to blame the victim occurs because of acontextualized knowledge. When you start to consider the effect of environments on people it becomes more difficult to blame individuals for their situation. However, for a project committed to critical consciousness and praxis, qualitative methods are not 
enough. Therefore, I root my inquiry in the tradition of participatory and action forms of research (see Brydon-Miller, 2001). Instead of eschewing the intervention my inquiry serves, I am proud of the fact the interviews I conduct are likely to further stimulate critical consciousness development in the participants. Further, the goals of this dissertation include the advancement of progressive social change.

In this dissertation, I have also tried to integrate aspects of participatory research methods into the study. I have sought participant input in resolving complex ethical dilemmas. Additionally, my preliminary research as a participant observer allowed me to integrate participants' insight into the development of the dissertation's methods and theory. Finally, I did not view my research participants exclusively as a form of data. Instead, I invited participants to help me theorize the processes under study.

This study's roots in social action, participatory, and qualitative forms of inquiry are carried into my methodological commitments at various levels of the project. Three of the levels occur with every Girl Power course with my research being an additional level unique to the quarter I observed. I identify the usual three interconnecting levels as the classroom experience, the rap sessions experience, and the mentor experience. The classroom experience consists of the activities and discussions facilitated by the instructor and the reading and writing students do outside of class to prepare for classroom sessions. The instructor integrates many aspects of Critical and Feminist pedagogies into the course. The mentor experience exists as a sort of intermediary between the instructor and students enrolled in the capstone. The mentors have previously taken the course and been identified by the instructor as students highly 
committed to the goals of Girl Power and as having a high level of feminist

consciousness. The quarter that I observed had three mentors from previous classes. One individual had mentored for the course multiple times and two mentors were new to the experience. The mentors received additional support from the instructor through weekly meetings, which I also attended. The mentors also provided additional support to students. At the beginning of the quarter, the students in the course were divided into three mentor groups, which met for one hour of in-class time per week to discuss the week's readings and discuss issues students might be having with the overall class experience (I attended the same mentor group throughout the quarter). The mentors were also in charge of reading and grading the reflection papers for their small group.

Another level of the project is the community engagement component of Girl Power. All capstone students are required to go into the community, in groups of two or three, and conduct rap sessions with a group of adolescent girls. These rap sessions, which drew on focus group methodology and consciousness-raising groups of the second wave of feminism, take different forms, depending on the facilitators and the participants. The Critical and Feminist pedagogies modeled in the classroom are encouraged as a way to conduct the rap sessions. In other words, the rap sessions are driven by the adolescent girls' experiences with capstone students' working to prepare multiple activities for each session. Every session is audio recorded and transcribed by a capstone student. The classroom and rap session levels of the course come together in the production of the Zine, the final product of the course. The course divides the transcripts from the rap sessions by theme and each student has a number of themes in which they create pages 
for the Zine (see Appendix E for an example page) based on the words given to them by the different rap session groups. The commitment to qualitative research is present in the use of the "data" from the rap sessions to create Zines. Action and qualitative methods are connected in that the rap sessions and the Zine combine to provide an arena for adolescent girls' voices. Finally, both the classroom and the rap sessions are participant driven.

My similar commitments to action, qualitative research, participation, and the intersection of these three areas facilitated my integration into the Girl Power project. While I was involved in most aspects of the project, my research questions helped to focus my attention. The following questions guided the dissertation:

1) How are emotionally and relationally significant Girl Power experiences related to of socio-political-cultural (SPC) consciousness development?

2) What tensions arise between the dominant culture and/or significant others' values and the values of the Girl Power capstone and how do these tensions move individuals toward or away from critical consciousness?

\section{Finding the Setting}

As I began searching for a location for the dissertation, I was interested in a setting that would be an ideal environment for critical consciousness development. According the to the literature this would require a setting that utilized service-learning and Critical Pedagogy (Landreman et al., 2007; Reason et al., 2005). I was also interested in a setting that utilized Feminist Pedagogy because of its connection to emotions and relationships (Boler, 1999). I identified the Portland State University's 
University Studies program as a fruitful starting point for my search. I was particularly interested in the University Studies senior capstone courses because they combine community engaged learning and aspects of Critical Pedagogy. I met with various members of the University Studies faculty, including the Assistant Director, to discuss the courses they thought best represented my ideal setting. A course that came strongly recommended, by all sources, was the Girl Power capstone. After meeting with the course instructor and sharing with her my action commitments and use of qualitative methods we worked out a plan for me to observe the Girl Power course.

\section{The Girl Power Capstone}

The Girl Power senior capstone course is one of the longest running and most successful capstone courses at Portland State University. Girl Power is a one quarter class worth six credit hours. The course content is interdisciplinary in nature but does concentrate on understanding issues of gender oppression, particularly those related to adolescent girls. The syllabus for the course describes it as an advocacy project-inprogress focused on connecting "Women's Studies scholarship in the academy with praxis in our community." The instructor is highly committed to Critical and Feminist Pedagogies and utilizes aspects of each, including: check-ins and check outs, group work, discussion, reflection, praxis, connecting with community, linking the personal and political, teaching the intersection of social identities, and a focus on voice.

Class time is spent building community through activities, such as daily check-ins and practicing skills necessary for conducting the service-learning component of the course (i.e., rap sessions). This practice involves doing self-reflection and activities, such 
as identity mapping, which facilitates student's ability to understand how broad sociopolitical structures influence them and the adolescent girls they work with in the community. Logistics of setting up the various community partnerships, making the Zine, and reporting to the class on rap sessions were a major topic of discussion in the course. About one hour a week is spent in mentor groups where students discuss subjects such as, internalized oppression and how it could impact working with adolescent girls. The mentor sessions also allow for time to discuss and reflect on the course material, which includes the texts Girls Studies and Reviving Ophelia and research articles focused on feminist scholarship about teenage girls, focus groups, and Zine publishing.

The community engagement component of the course has students "coordinate a series of *rap sessions* with local teen girls about current issues in their lives" the syllabus continues "we will use these group conversations to encourage the girls to become part of our Zine project - where they write, edit, and publish a grassroots, mini -magazine with our class." The rap sessions are like focus groups that at least two class members co-facilitate. The focus groups bring together the class participants and teenage girls from a variety of local organizations. The focus groups allow teenage girls a space to talk about what is important to them and express their voice.

\section{Preliminary Research}

After locating the course, I engaged in preliminary research by becoming a participant observer in the capstone. My role as participant observer allowed me to better describe and understand the experience of the capstone students. I was also able to build rapport with the capstone participants. The observations aided in my development of the 
methods and theory for the dissertation. Through my preliminary work, I learned that interviews would be necessary to truly get at students emotional responses to the course. Additionally, the observations aided in the construction of the interview guide. More information on the course and my experience as a participant observer is presented in the next chapter.

\section{Participant Observation}

Participant observation is a method of data collection that allows the researcher to use her or his knowledge and experience in the setting to aid in the collection and analysis of events (Kidder \& Fine, 1997). As a participant observer, I wrote field notes during the observation of class time, during mentor meetings, and following rap sessions I co-facilitated. The field notes primarily focused on my reaction to the course material and discussions. Because reflections and dialogue are important aspects of a Freirean model of education and critical consciousness development, they were a fundamental focus of my observations (Freire, 2000). Yet, the latent level of analysis goes beyond what is spoken. Along with recording the verbal reactions of capstone participants I attended to the bodily reactions of students. For example, I noted if participants were shifting uncomfortably or nodding in agreement.

I gained access to the setting by meeting with the instructor and discussing my dissertation with her and what my role might look like if I was to engage in participant observations during the capstone. We decided it would make sense for me to be a true participant observer. In this role, I attend all class meetings, did the course readings, engaged in mentor sessions, participated in course discussions and activities, and 
conducted Rap Sessions. During these times I also kept field notes, recording important discussions as well as my and others reactions to the course. The class is a very intimate setting and involved a great deal of personal sharing. During the more intense discussions when students shared personal stories, I did not take field notes. I did this to respect the situation and so I could offer the person my full attention.

On the first day of the course, I told students what I was studying and why I choose the Girl Power capstone as the setting for my dissertation. The students seemed genuinely excited about the opportunity to participate in such action-oriented research, consistent with Stein and Mankowski's (2004) argument that one of the primary reason's individuals choose to be involved in research is to "help others and help science" (p. 23). Although this study does not meet the full criteria of a participatory study, I offered students the opportunity to have input into the development and interpretation of the study. Additionally, I tried to constantly find ways that I could give back to the capstone community. For example, I offered to meet with students after the capstone and talk with and/or assist students interested in graduate school and/or research.

I felt that my presence in the class aided in the rapport I built with students, which was necessary to conduct interviews that were quite personal and occasionally sensitive in nature. Further, my commitment to the safety of the space and my shared commitment to valuing women's voices (a major goal of the Girl Power capstone) drove aspects of my methodology. I worked with capstone participants to co-develop the informed consent process and form (see appendix F). Further, my commitment to the participants continued in the form of obtaining their input at various points throughout the research 
process, including: reviewing interview transcripts and sharing findings with them. In line with participatory research, I not only acknowledge the role I have in the setting but embrace it (Tolman \& Brydon-Miller, 2001).

My role as participant observer was also beneficial in that I went through the same experience as my participants, which helped prepare me to interpret the findings of the dissertation (as suggested by Adler \& Adler, 1987). Additionally, formalizing my experiences in the course offered an additional point of comparison. Such multiple roles are often viewed as a strength in participatory action research (Tolman \& Brydon-Miller, 2001). The complexity of the situation allows for increased critical consciousness for all involved parties and for my understanding of this process. While this complexity and my research methods precluded my ability to make cause and effect claims regarding what did or did not cause the development of critical consciousness, I am not limited in my ability to gain an understanding of how participants experienced the process.

\section{Interviews}

I chose interviews as a method of data collection because of their fit with the qualitative research questions posed in this dissertation. Semi-structured interviews are ideal for phenomena that are personal and/or complex (see Smith, 1995), such as critical consciousness and emotions. Further, this dissertation ventures into the understudied area of emotions and relationality in critical consciousness development. Because the study sought to increase understanding in this domain, an interview strategy that allowed for some openness and depth of probing was necessary. Interviews also allow participants to have a one on one conversation with the researcher that are less directly 
affected by group dynamics. Further, interviews allow the researcher to probe topics that participants might not want to discuss in a group setting.

These interviews were semi-structured in nature and focused around understanding participant experiences in the course, their affective transformations, and how their thoughts, feelings, and/or behaviors have changed post capstone. Memories that are emotionally significant are more readily recalled than memories that are not tied to emotions (Haaken, 1998). Further, memories that are associated with emotions tend to be stronger (Haaken, 1998). This reflective design offered a multitude of advantages for the issues of concern in my dissertation. First, looking back on the course students are likely to have a better understanding of the moments and experiences that had the strongest impact on their critical consciousness development (Landreman et al., 2007). Hochschild (1982) uses symbolic interactionism to suggest that the initial performance of an emotion for others (such as occurs in an emotionally evocative course) is superficial and involves surface acting; it is only later that the initially performed emotion may become a more substantial emotion that involves deep acting. Second, by asking participants to engage in reflection and discuss their involvement in action I am continuing the circle of action and reflection that constitutes the core of praxis and process of critical consciousness development, therefore contributing to the action component of the dissertation. Third, by utilizing the time lag between the end of the course and the interviews I was better able to attend to lasting impacts of the course and begin to explore the connect (or disconnect) between development of critical reflection and involvement in critical action. Fourth, the interviews that are necessary to get at the 
issues of interest in this study are relatively personal and sensitive. It is unlikely that I would have been able to conduct (and be confident in my results) this type of interview without the rapport built through my participation in the course. The design of the study does not allow for complete confidence in the course being the only or even primary cause of critical consciousness change but by rooting the interview in discussing the course and the associated experiences, I aim to parse out what aspects of the course participants identify as contributing to their SPC consciousness development.

After multiple attempts and contact through email and phone over a two-month period I ended up with 10 participants. Interviews lasted between 45 and 90 minutes. I asked participants to bring in copies of artifacts produced from the course for example, reflections, Zine pages, or artwork. Having the students bring the artifacts grounded them in the capstone experience and their affective reactions to that time. The use of artifacts served a projective function in helping students express the more affective dimensions of the course without being specifically prompted. I also asked participants to discuss their capstone experience more directly. I constructed a timeline (see appendix B) of important course events from my field notes and I asked participants to talk through their emotions at different points in the course using the timeline. I also amended my interview protocol after my first few interviews and had participants write down emotions and or faces on the timeline as well. I began the interview very generally by letting students talk without much guidance and then moved to prompt for more specific information about their experience. 
The order of the interview guide (see appendix A) is designed to start with very general questions regarding participants' experience in Girl Power. The first question is open to allow participants the opportunity to discuss the aspects of Girl Power that were most meaningful to them, without being initially prompted by more directed questioning. The second question involves participants in a process of projecting their course experiences onto artifacts they produced in the course (e.g., Zine pages, reflection papers, and/or art). After this the questions become more focused as I provided students with a timeline of events in the course that my field notes indicated were significant and/or I theorized might be noteworthy.

In question four, I moved to begin discussing SPC consciousness directly. I did this by introducing a quote that the instructor used on a regular basis throughout the course and asked participants how they understand the quote. Again, I tried to start with an open question before probing students more specifically. In question five I began to probe more directly into how participants understand SPC consciousness, I use the language of feminist and social justice consciousness because this is what was introduced and used throughout the course. When going through this question I asked students to relate this growth to specific course experiences.

At question six the interview changed in nature. After allowing participants to talk openly about their experiences in the course and SPC consciousness I moved to ask more specific questions based on my theory of SPC consciousness development. I began by asking students why they signed up to take the Girl Power course originally. Next, I included a question designed to investigate the emotional energy in Girl Power. I then 
asked about collective identity and community post capstone, as my theory predicted in order for critical consciousness to be maintained engagement in a critical community must continue. Question nine asked directly about participants' interpretation and reaction to popular culture following the Girl Power course. Question 10 offered participants the opportunity to address my project aims directly by asking them if they think Girl Power "works" in creating critical consciousness. The next few questions ask specifically about different aspects of the course that the instructor, mentors, and my field notes suggested might be important to ask about. Question 14 asked students directly if they felt any conflict between what they were learning in Girl Power and other aspects of their life. I concluded the interview by asking students if there were any significant Girl Power related events, which had not been discussed. I conducted a practice interview with one of the mentors to fine-tune the ordering and language of my interview guide.

These interviews served as the primary data analyzed for this dissertation. The interviews were audio recorded and transcribed. After each interview, I sat down and wrote a reflection of the interview and tracked my subjective responses as well my developing understanding of the phenomenon. An undergraduate research assistant and I transcribed the interview verbatim using Express Scribe. I then listened to each interview while reading the transcript and adding potentially important emotional responses (e.g., sounds unsure, laughing, increases in volume, pauses). I hope that by both asking students to talk about emotions and looking for reactions that may indicate an emotional response I was better able to gain a picture of the emotional and relational processes involved. Only when the transcripts reached this level of detail did I begin the process of 
formal analysis, detailed below. The level of transcription originally included pauses, tone changes, and verbal cues (e.g., laughing), which were included in double parenthesis. When presenting quotes from participants, these notations have been included only in the quotes where I use them to aid in the interpretation of the quote. Additionally, italics represent participants' emphasis unless otherwise noted. I felt it was important to include this level of detail in the transcriptions because of my focus on emotions, which are not always conscious or readily articulated.

\section{Sampling}

I selected the course using criterion sampling, choosing a course ideally designed to develop critical consciousness. I contacted all capstone participants multiple times through email and phone calls. Ten capstone students were interviewed. Of the six women that were not interviewed four did not respond to any of the prompts and two initially responded but never got back to me with times they were available, though I prompted responses by email and phone multiple times. It is hard to know why these students choose not the respond or follow up with my request for interviews. Some students may have been or felt too busy to make time participate in the study. Of the non-responders, three were individuals that were relatively reserved during the course. This finding is based on my observations and thus I cannot make inferences as to why this subset of students chose to participate less actively in the course.

\section{Ethics, Confidentiality, and Informed Consent}

Serrano-Garcia (1994) defines ethics as a theory that helps a community clarify its values. Traditionally sub-fields of psychology (e.g., Clinical and Social Psychologies) 
have relied on a professional code of ethics as outlined by the American Psychological Association's (APA) code. This code was developed in the language of client-therapist model, traditionally used in clinical psychology settings (Trickett, 1998). The language thus suggests that the relationship between researcher and research participant mirrors the client-therapist relationship with information flowing from the client to the "objective" therapist. While this paradigm may be sufficient for social psychologists working in a controlled laboratory setting with individuals it is insufficient to deal with the issues faced by community psychologists.

Complicating the ethical issues faced by community psychologists is their work with whole communities of people, as opposed to working with individuals. Further complicating the issue is the tendency for community psychologists to strive for a twoway flow of knowledge between researcher and participants. This adds numerous avenues for potential value conflicts. My commitment to participatory methodologies and my research participants leads me to integrate them into the research process. I integrated the participants' values into the informed consent process. As the nature of the course is quite intimate and sensitive but focused on voice, constructing the informed consent process was complicated. To use the traditional anonymity or keeping participants identities concealed and a response de-identified, protects students, but also deprives them of their voice. On the other hand, to allow participants to attach their name to their responses puts them at potential risk because of the sensitive and intimate nature of some of the material. Because of this conundrum, I discussed these various options with the class and we decided that everyone would pick their own alias, which is what the 
adolescent girls participating in the rap sessions do. Before each interview, I went over the informed consent document with students and obtained participant's signatures and chosen alias.

Additionally, I allowed students multiple chances to review the research. Each participant had the opportunity to review their individual transcription to make any additions, clarifications and/or remove any material felt to be objectionable. That said, no participants chose to make any changes to the interview transcript. In addition to increasing the trustworthiness of the study (Guba \& Lincoln, 1985), involvement in the research process offers an additional advantage for the participants. Many of the students in the class were interested in research and graduate school but are limited in their knowledge of these issues. Involving students in these various aspects of the research offered the opportunity for them to learn more about qualitative research and some of the aspects involved in obtaining a graduate degree.

My findings do not include a biographical sketch or summary of the individual participants. As I was analyzing the data and preparing to write the findings sections I began to have concerns about participants' confidentiality. It is important to me to share my results with the Girl Power community, but I worried that participants' could be identified based on the personal nature of the data that was shared in the interviews. To prevent this possibility I do not include full interview transcripts in the dissertation document. When a particularly personal or sensitive piece of data is essential to the understanding of my analysis and/or findings, I have included said data but I have either omitted or changed specific details (e.g., locations) to ensure the narrative is not lost, but 
remains unidentifiable. This concern also limits my ability to discuss how participants' race, class, and/or sexuality may have influenced their experiences. Additionally, my ability to discuss this aspect of the findings beyond a certain level of depth was inhibited by the low number of participants' I have in any one demographic category. At times when it is important to the analysis, I might state that a person is a member of a marginalized community, besides being a woman, but I will not state what specific identity I am referring to. While limiting, I believe taking these precautions helps maintain participant confidentiality and it keeps me from overstepping the claims I can make with this limited sample size.

\section{Data Analysis}

I analyzed the interviews using Carol Gilligan's Listening Guide (Gilligan et al., 2003). The Listening Guide provided a helpful analysis tool for the dissertation in a number of ways. First, the Listening Guide was developed through a process very similar to the capstone course. Gilligan and colleagues developed the guide through their research with pre-adolescent and adolescent girls, specifically focusing on their voices. This process mirrors the structure of the course, as the capstone instructor and mentors facilitates activities focused on increasing women's access and comfort in using their voice and then the capstone students go out into the community and do the same for younger women. Second, Carol Gilligan's theory of women's development offers a unique understanding of how women and girl's experience, particularly concerning moral development and how it relates to relational dynamics. Third, the Listening Guide accounts of this relational sphere by rooting the interpretation of qualitative data in the 
relationship between the researcher and participant. This method of collecting and analyzing qualitative data values participants voice and the relationships involved in the process of data collection and analysis. Gilligan and colleagues (2003) said about the Listening Guide:

...assumptions include the premise that human development occurs in relationship with others and, as such, our sense of self is inextricable from our relationships with others and with the cultures within which we live...The Listening Guide provides a way of systematically attending to the many voices embedded in a person's expressed experience. (p. 254)

The relational nature of the Listening Guide matches the relational and dialectic conception of consciousness and emotions utilized in this dissertation. The guide allows the multiple voices within the interviewee to be accounted for and analyzed in relation to one another and in relation to others.

The extension of the use of the Listening Guide into the area of critical consciousness offers an exciting and new terrain for discovery. Gilligan et al. (2003) offers an overview of the Listening Guide:

The Listening Guide method compromises a series of sequential listening, each designed to bring the researcher into relationship with a person's distinct and multilayered voice by tuning in or listening to distinct aspects of a person's expression of her or his experience within a particular relational contexts... Each of these steps is called a "listening" rather than a reading, because the process of listening requires the active participation on the part of both the teller and listener. (p. 159)

The first two listening steps are rather open and fluid, listening for what the participant is communicating with an open mind. In these listening the participant's voice and experience is privileged. The focus of the last listening and analysis centers on the research questions and theoretical frameworks driving the project. It is in this last stage 
of analysis than I began to interpret the data I collected through the theoretical lens of the dissertation.

The first listening has two parts-- "listening for the plot" and for "the listener's response to the interview" (Gilligan et al., 2003). Practically, this means noting "what stories are being told", what contexts are they being told in, what individuals are involved, what are the "repeated images and metaphors and dominant themes", and what is the relationship between these different aspects of the different stories (Gilligan, et al., 2003, p. 257). For this listening, I read the interview transcripts and used the qualitative data analysis program, Dedoose 4.5.95 web application, to code re-occurring motifs within the data. At the end of this step I produced a written reflection of what I was hearing as I interacted with each interview transcript.

The second part of the first listening involves identifying the relationship between the researcher and participant and involves the researcher tracking their emotional reactions to the stories that are being told. As I read the interview transcripts, I traced my own subjective responses to the data using memos. In addition to tracking my reactions to the material, I attempted to integrate how my social location interacts with that of my participants in ways that construct what aspects of their experiences I do and do not have access to and the power imbalances that affect what and how participants tell their stories. After completing the first listening on all ten interviews, I wrote up a brief synopsis of my initial reactions and interpretations of the interviews as a set.

The next listening is when the process becomes more focused on the posed research questions and utilizes the developed theoretical framework. This step occurs last 
so that the previous reading can provide context for this last step of interpretation.

Within this step, the interview is read multiple times each instance attending to a different voice in the transcript. The idea of each of the readings in the last step is to think of and define a possible voice and then look through the transcript for support of the voice. Gilligan and colleagues (2003) describes this listening as:

We specify the voice we will listen for and determine what the markers of a particular contrapuntal voice are or, more simply, how we will know this voice when we hear it. The text is read through listening for just one voice at a time, and the appearance or evidence of this voice is underlined in a color chosen to mark it. Reading through the text a separate time for each contrapuntal voice allows for the possibility that one statement may contain multiple meanings, and therefore may be underlined multiple times, and also allows the researcher to begin to see and hear the relationship between the person's first-person and contrapuntal voices. (p. 263)

These voices may have a variety of relations to one another, some harmonious, some in opposition, and some contradicting one another, thus allowing for an increased understanding of the highly complex and contextual nature of psychic phenomenon, like critical consciousness.

Before beginning the last reading, I revisited my dissertation research questions, literature, and theory section. After reviewing these documents, I began to review the codes I had developed from the first listening as well as the memos and summaries I constructed throughout the initial stages of data analysis. During this period I dropped some initial codes, combined some codes, and renamed some codes based on the knowledge of the previous analytic passes and the reviewed literature and theory. I also used my research questions to concentrate on the codes mostly directly related to the phenomenon of interests for my dissertation. Practically this meant putting aside some 
codes that deal exclusively with the educational component of the project (e.g., ideas for improving Girl Power). While these codes are potentially useful for future study, they stray from the more psychological focus of the present dissertation.

After reformatting the initial codes from the first listening into themes, I engaged in an additional reading of the transcripts utilizing the finalized codes to make sure all relevant sections were appropriately coded. This was necessary not only because of the changes to the code process but the iterative development of themes meant that some codes were developed after my initial pass of coding some of the early interviews. Additionally I underwent a process of drawing out different potential relationship between the codes, themes, and interview summaries as they related to my research questions. This involved creating multiple sketches of my developing understanding of the relationships among the data as well as debriefing with other qualitatively trained graduate students and my dissertation chair. Following this process, I was able to identify the two themes I concentrated on in the last step of the Listening Guide. These two themes are addressed in the next chapter, which presents the findings of the dissertation. 


\section{Chapter Five: Findings}

In this chapter, I address the findings of the dissertation. I begin by presenting information on the Girl Power course, which provides an overview of the daily activities of the course and its main components. These data were obtained through course materials (i.e., syllabus) and the notes I collected as a participant observer in the class. The overview of the course provides an important context for analysis of the interviews.

Next, content from the interviews is organized around the two themes that emerged in the interview transcripts--processes of awakening and sources of dissonance. The material in the processes of awakening theme addresses the first research question: How are emotionally and relationally significant Girl Power experiences related to of socio-political-cultural (SPC) consciousness development? The material in the sources of dissonance theme addresses the second research question: What tensions arise between the dominant culture and/or significant others' values and the values of the Girl Power capstone and how do these tensions move individuals toward or away from critical consciousness? These themes arose through an iterative process of attending to the content present in the interviews, applying my theoretical lens to the data, and attending to my research questions. The section for each theme includes illustrative quotes as well as brief discussions and questions raised by the quotes. Quotes are identified by the selfchosen alias of each participant. This chapter focuses on presenting my findings in a descriptive format. The broader discussion and the theoretical implications of the findings are discussed in chapter six. 


\section{Overview of Observations in the Girl Power Course}

Past research has tended to eschew the role of emotions and relationships in conceptualizing processes of critical consciousness development. The Girl Power capstone course focuses heavily on emotions that arise in the course and building engaged relationships. I have theorized each of these components as central to SPC consciousness development, making Girl Power an ideal site for inquiry on the development of critical consciousness.

The course included 17 participants, and all but one was female. The majority of the participants were white, as well as several African-American, Asian-American, and Latina participants. In addition, a number of the students identified as lesbian, gay, and/or queer. Many of the students were traditionally college aged, although a few students were non-traditional in their age and life experiences. For some the content in Girl Power was surprising and for others it repeated or reinforced ideas they had been exposed to in previous classes or in life experiences.

My experiences as a participant observer in the Girl Power course enhanced my belief that the development of critical consciousness involves understudied emotional and relational components. Indeed, emotion is invoked early on in the course as central to the academic experience. When describing the Girl Power philosophy, the instructor often states, "it is approaching the cultural lie in really a courageous way." In this statement, the focus on courage includes an implicit affective dimension, particularly in the aim of overcoming fear. 
Although the course was centered on discussing women's issues, the instructor took an intersectional approach to the topic, often discussing the unique experiences of women of color and/or LGBTQ identified women. The course material was evocative and challenged students' ways of viewing the world. For example, students were asked, through activities like social identity mapping, to label the aspects of their identity that granted them privilege and to begin to understand how this privilege affected their viewpoint and experiences.

Class was held in a circle so that everyone could see each other. When the class session began, the instructor closed the door "to protect the energy of Girl Power." Some aspects of the course had a ritual feel, such as the routine of always beginning with a check-in. Other aspects of the course differed from day to day and changed as the quarter moved along. The instructor occasionally stood in front of the class and used the board to present information, but this was relatively rare. Most of the class time was spent in discussion rather than lectures. In the beginning of the term, only a handful of students participated in discussion. But as the course developed, students contributed to discussion on a regular basis. However, a handful of students remained relatively quiet throughout the quarter.

One of the first activities conducted in the course was what the instructor called co-creating our classroom community. This activity involved the class jointly coming up with the norms and procedures that would facilitate a safe space for learning and growth. These norms ranged from respecting and listening to one another to the decisions to rotate bringing food and drinks to each class to facilitate a feeling of community. This 
activity set the stage for the rest of the term by encouraging student input into the course and stressing the importance of having an open and respectful space for discussion.

Looking at Girl Power. Each session began with a check-in that consisted of a question, sometimes serious (e.g., "How will your own struggle with internalized oppression impact your work with teenage girls?") and sometimes relaxed (e.g., "If you could have any super power what would it be?"), to which everyone in the course responded (although students always had the option to not respond or pass). Sometimes this activity was relatively quick, lasting only 10 minutes, and at others times it would take an hour or more. Check-ins served the purpose of facilitating the process of getting to know one another and feeling comfortable in the classroom.

For the first few weeks of the course, following completion of the check-in, the remaining time was often spent on course tasks related to rap sessions, including coming up with and contacting organizations in the community to hold rap sessions, brainstorming possible rap session formats, and conducting mock rap sessions. These rap sessions were a major component of the course. The groups of two or three students, who worked together throughout the quarter, went out into the community and conducted sessions with teenage girls at consenting community organizations. Rap sessions were modeled similarly to the course. Although student facilitators prepared art, craft, discussion, and reflection activities, the sessions were driven by the needs of the participating adolescent girls. One popular activity for rap sessions was the question box. In this activity, the facilitator brings a box, usually decorated, to the rap session and the girls write whatever questions they would like to talk about on a slip of paper and drop it 
in the box. At each session, a facilitator draws out a question, reads it aloud, and opens the floor for discussion. This activity invited the girls to discuss topics important to them. The rap sessions were audio recorded and later transcribed. These transcriptions were then divided into themes, which became the raw material for the Zine. The sessions seemed to not only cement friendships but also to enhance the understanding of course material for students, a tendency the instructor suggested happened in many past Girl Power courses.

Once the rap sessions were underway during the term, class time was spent in rap session report outs, where each group that had conducted a session that week would describe the group and reflect on their experiences. Report outs also allowed students to troubleshoot any issues they were having in the rap session, while other class members, mentors, and the instructor offered potential solutions to perceived problems. If time allowed, or if class had been especially intense or evocative that day, a check-out would be conducted at the end of the class. This check-out was usually either pleasurable or focused around getting a sense of how everyone in the room was feeling. Check-outs served as a way of debriefing students before returning to the world outside Girl Power.

On the second of the scheduled sessions each week, the class would begin by breaking into small mentor lead groups without the presence of the instructor. Each group was facilitated by a mentor — undergraduate students who had previously taken the course and done well, as well as having demonstrated commitment to the course goals. These groups lasted about one hour and served as the place to talk about the assigned 
reading. Journal writing based on the assigned readings were also due at the beginning of this session.

I attended and participated in the same mentor group throughout the quarter. The decision was made in consultation with the instructor. We believed that if I rotated groups throughout the term my presence might disrupt the intimate nature of the groups. More often than not, this time was spent in personal sharing that was sometimes related to course readings and sometimes not. It seemed that often the mentor was the only person that had done the assigned readings. Students often had very little to say regarding the readings, when probed about their silence some admitted to not having done the reading. To keep participants engaged the mentors came prepared with discussion topics that did not necessarily depend on having completed the readings. It was not uncommon to see individuals in mentor groups laughing and crying together as they shared personal experiences and struggles.

The remainder of this second weekly class session was often dedicated to class activities, such as the identity circle or learning about and practicing interruptions. Activities such as social identity mapping and the identity circle had the aim of helping students to integrate personal experiences into their understanding of social, political, and cultural structures. These activities utilized the consciousness-raising tools of the women's movement to make the personal political. The identity circle was a particularly powerful activity that stirred personal histories. The activity had the entire class stand in a circle and take turns making a personal statement about their past experiences and stepping into the circle. Other individuals that had experienced something similar would 
step into the circle as well. Others could also step in as allies. While the activity started with relatively innocuous statements, such as "I played sports in high school", it moved to be quite intense as some students began to describe histories of sexual assault or selfharm.

The instructor espoused a very specific type of critical action she termed interruptions, devoting one full period to instruction on this principle. She conceptualized interruptions as the process of stopping someone that is engaging in an oppressive action and/or statement. The interruption in which someone might engage may be simple, such as telling the person that they have said something racist and then leaving the situation, or more complex in engaging in more in-depth conversations with the person, telling them how their action or statement was oppressive and how it hurts others. The Girl Power capstone spent considerable time talking about and identifying oppressive language as the sites of interruption strategies. The course session that focused on interruptions offered students the chance to role-play and practice strategies, including emotions that arise in carrying out specific strategies.

During the last part of the quarter, as rap sessions were concluding, the second half of class on each day shifted to focus on the process of creating the Zine. The Zine is produced by Girl Power course members and includes the artwork and words of the rap session participants. All class members and rap session participants received a copy of the finished Zine. Creation of the Zine involved a number of steps, including coming up with a title, deciding upon themes, assigning the themes to different individuals in the group, and ordering the final version of the project. Each of these tasks was carried out 
in a participatory fashion. While the Zine itself was an important physical artifact created from the class, and many participants discussed this project in their interviews, it was also a site of frustration and tension. As my findings illustrate, participants often struggled with the process of making decisions about the Zine in the face of looming deadlines.

Time near the end of the term also focused on preparations for the Girl Power party. This was a class gathering at the end of the term that included many of the girls that participated in the rap sessions in the community. Preparations for the party included soliciting food donations and coming up with activities for the girls for this special event. Preparing for the party and the Zine during the last few weeks of the course meant working against a strict deadline for the end of the term.

The range of reactions to the course was striking. Some students, many of whom had previously taken Women's Studies courses, were highly talkative and involved from the beginning. Other students became more open as the course went on. A handful of students started the course as seemingly closed off and reserved but after a few weeks became the most active during discussions. A few participants remained quiet throughout the term, however, never appearing to form friendships in the class and often choosing to pass on check-ins and discussions, even when asked for their thoughts directly by the instructor. Smaller friendship groups tended to form among individuals who participated in the same rap sessions.

The instructor exerted a powerful influence over the experience of students in the course. She was consistently described by students as upbeat and known for her positive energy and her caring attitude. She was viewed as both as a facilitator of consciousness 
development and a role model, embodying many of the principles of critical consciousness.

During my observations, it seemed that the emotions and relational dynamics of the course allowed the process of SPC consciousness development to unfold. To understand if, how, and why Girl Power develops critical consciousness, individual interviews provided the method to explore participants' experiences and emotional responses. In the following sections, I present the findings of the interviews I conducted with the 10 consenting capstone participants. These findings are divided into two themes, processes of awakening and sources of dissonance, each of which includes multiple sub-themes.

\section{Listening to Girl Power: Processes of Awakening}

The metaphor of "awakening"—as a central theme-emerges from descriptors enlisted by participants themselves in characterizing what many view as a dramatic learning experience. The theme is described as a process because many participants view their awakening as an ongoing commitment rather than a discrete academic experience based on traditional learning objectives. By combining these two terms, I suggest that the Girl Power course is a part of an ongoing process of coming to understand the world in a new way. By pluralizing the term process, I bring attention to the multiple dimensions of this awakening. Although participants sometimes describe their developing consciousness as a sudden transformation, the general tendency is to describe the "awakening" as a gradual process. This theme connects to the first research question: How are emotionally and relationally significant Girl Power experiences related to SPC 
consciousness development?, in that this theme explores participants' self-described transformation during and as a result of the Girl Power course.

Participants also describe their processes of awakening in ways that mirror a religious conversion experience. This is evident in the language that participants use and their overall description of the processes. I attempt to separate the evangelical impulses of the course from critical consciousness by focusing on the aspects of critical consciousness identified in the theoretical framework. Of central importance to this separation is the political nature of critical consciousness development (Freire, 1993). Further, critical consciousness encompasses respect for differences of opinion and tolerates ambivalence (Haaken, et al., 2011); while the more evangelical impulses of participants often engage in binary good/bad splitting of a fundamentalist nature. Critical consciousness enhances an individual's ability to lead and create his or her own path (Watts et al., 2011) whereas a fundamentalist conversion experience takes the form of accepting authority and following an already set path. Despite the vast differences in the results of a conversion experience and critical consciousness development, the processes seem to share some similarities. Both processes involve a struggle to integrate new and old ways of seeing the world. Further, both experiences often involve new experiences that rouse intense emotions and passions in individuals. These energies can then be applied in a regressive or progressive form.

Many participants employ terms or metaphors, typically visual metaphors, to convey the idea that the course exposes them to a formerly concealed reality. In this respect, the course represents a kind of conversion experience. Participants speak of this 
process as "seeing things in a new way." Violet states, "oh my god, I felt like I lived in a box or something." Violet compares her changing view as akin to coming out of darkness, suggesting that her previous way of looking at the world was shrouded in a lack of knowledge, mystery, and isolation. Ruby tells her own story of transformation: "I went in with a whole new perspective... and I was able to just have more patience... that [the rap sessions] just woke me up." Ruby's metaphor—-that she "woke up"—conveys a sense of emergence from a previously foggy haze of sleep. After exposure to other people's stories through the rap sessions, she now has a more clear view of the world. While the quotes invoke dramatic ruptures in understandings of the world, participants also emphasize that such changes develop gradually over the course of the term. The emergence of a new lens on reality illuminates previous ways of seeing things as distorted. Most participants discuss this awakening as something that is an ongoing process with no defined starting or ending point but rather as a continuing journey toward greater knowledge about the world.

When discussing her transformation, Samantha repeatedly refers to visual perception in her choice of metaphors, "When you take classes like this, it's like someone just peels layers off of your eyes. And you're like, oh my god this is horrible... you notice a lot more of it [sexism]." Samantha's use of this violent metaphor also hints to the painful feelings Samantha may associate with her process of awakening. Her choice of such a graphic and disturbing phrase suggests that the process she is describing is infused with some amount of pain and suffering. Samantha does not attribute this process to herself. Rather she locates the action of this seemingly painful experience 
outside of herself, "its like someone just peels layers off of your eyes" [my emphasis added]. Samantha's quote suggests that the process of awakening may not be selfdirected and may be forced by the hands of another.

The role of the Girl Power course in this awakening varies. For some, the Girl Power experience provides the impetus and for others it adds to their previous experiences both within and outside the classroom. For the latter students, the awakening does not begin but continues in their encounter with Girl Power.

\section{The role of Girl Power in the process of awakening. The Girl Power} experience cannot be understood in isolation. Participants come into the course with varying levels of knowledge and experience. In order to understand the role of Girl Power in moving students along the SPC consciousness continuum it is important to consider how the course fits into their broader arc of experience. By suggesting that awakening is an ongoing process, participants allow room for disappointment in both themselves and the course. This allows space for participants' disappointment when they fail to meet the criteria they have identified as necessary for living life in a less oppressive fashion. As participants fail at times in living up to the standards they set during Girl Power, as discussed more in later section on dissonance, by acknowledging their political awakening (which includes changes in thoughts and actions) as a process they are able to deal with the times when they fall short of the ideals developed through the course.

Elizabeth had a fair number of experiences in previous courses that focused on developing a critical perspective. She does not speak of Girl Power as changing her 
awareness. Nevertheless, she does suggest that the experience was profound for other people:

Whether or not the goal was to enhance that [feminist consciousness] for [rap session] girls or for the class [participants], I don't know, but I think it definitely works for the class... a lot of people don't get those kind of things and I feel like it should be a requirement. But I think it's huge for a lot of people, I think it's really eye opening. Especially people that aren't in the social sciences or anything, and they don't ever get to talk about any of this.

Like many other participants, Elizabeth uses a visual metaphor to describe her awakening experience, suggesting again that the way that she "sees" the world has changed.

Elizabeth seems to be saying that the course is particularly useful for those that have not been exposed to these ideas before, but the unspoken aspect of the quote suggests she believes the course is less valuable for those that have already developed a critical analytic capacity. Elizabeth may argue this because it is similar to her past experience in classes. Alternatively, she may be trying to speak highly of Girl Power even if she cannot do so from her own experience. Elizabeth may be responding to a demand characteristic attached to the course and/or the interview, however, in conforming to the expectation that Girl Power is a powerful or transformational experience. The possibility of responses driven by a demand characteristic of the course and/or interview is noted throughout the data. The Girl Power experience seems to place certain expectations on participants that they have a dramatic transformational experience that results in them becoming more feminist in orientation. My presence in the course and the style of questions I asked in the interview may have perpetuated this demand characteristic, which places limits on the full range of experiences and emotions available to students during and following the course. 
Dita also has taken "a few other feminist classes" but states that Girl Power was still a powerful experience for her. For Dita, Girl Power added something that was missing from her previous Women's Studies courses:

... with other classes any time that there was even discussions about such subjects [oppression] it was always in such a scholarly studied way that you're writing up reports about really horrible things, but it's almost disconnected from you because you're not thinking about it on a personal level or about how it affects other women in your life ... so that's where I had the biggest break through in Girl Power was like, oh yeah it's real people and it should matter to me.

She had learned about oppression and specifically sexism before, but the lack of emotional and relational content in the courses seemingly prevented her from using or acting on this knowledge. Dita suggests that a strength of Girl Power, as opposed to other Women's Studies courses, is that it allows room for experiential knowledge and learning. However, the strengths of Girl Power in this area also suggest certain limitations. While emotion and thinking are both important to the process of development, the integration of the two involves a delicate balance. There is the possibility of falling too much on one or the other of this thinking/feeling divide. In a course that values the affective side of learning, there is the possibility that emotions may overtake critical thinking, a concern that led to Freire's tendency to discount the role of emotions in his theorizing on critical consciousness development.

Violet discusses her process of SPC consciousness development going back to childhood:

I guess what it [Girl Power] did was sort of make all those thoughts that I already had more cohesive and understandable.... And even if you're raised in a house, like I was, that did not promote racism or sexism you still get it from other places. And it's some kind of weird brainwashing that happens, just being a member of society... beforehand it was like, more fragmented and then after taking this class 
I was able to sort of like bring together everything that I've ever learned from other people about social justice, put a title on it and make it more cohesive.

For Violet, Girl Power is useful in that it helped her acquire a vocabulary to describe her past experiences and current thinking. It seems that for Violet the more cognitive and scholarly aspects of the course - learning the language and theory of oppression - were the most useful in continuing her process of awakening. In enlisting the term "brainwashing," Violet emphasizes the power of oppressive ideas, although she locates their source in the larger society rather than in her immediate familial or social world. For Violet, the Girl Power experience is framed in alliance with the progressive ideas in her own family experience, set in opposition to broader cultural forces of oppression. Girl Power is not the primary impetus for Violet's process of awakening but she did enlist the course to reframe earlier life experiences.

Many participants highlight the idea of process by stating that they hope that their consciousness continues to grow following the course. Mint Tea states "...I hope that it [feminist consciousness] will change and I will be able to do more and apply to social justice more because it makes you feel good about yourself when you're giving back." Mint Tea recognizes the work that needs to be done to create long-lasting social and political change and that this work needs to continue past the end of the course and is not based solely on self-less acts but also carries self-oriented interests. The work of fighting on behalf of others can offer ego recognition, which can protect against burnout by offering immediate personal rewards. Ego recognition can also help individuals to sustain in critical action against seemingly insurmountable odds because the action in and of itself feels meaningful and good. Other participants also espouse the idea that 
engaging in interruptions has personally fulfilling aspects. However, too much focus on self-realization can also become limiting. Long-term social justice work involves an integration of aims oriented toward fulfilling personal needs and helping others and reaching group goals (Cipolle, 2010).

Overall, it seems that Girl Power provides different things to different participants based on their cognitive and emotional needs as well as past experiences. Participants seem able to draw what they can from the course to contribute to their process of awakening. I now turn to the emotionally significant aspects of the course and how emotions may drive or inhibit SPC consciousness development, as suggested by my first research question.

Emotions and the processes of awakening. The experience of adopting a new vantage point and rejecting old worldviews is described by participants as an emotionally intense and complex. Participants identify a broad range of feelings as central to their process of awakening. Some of these represent complex concepts that are not easily subsumed under any one category, including emotion.

Dita discusses the many emotions involved in her process of awakening:

...just one individual who says something that is oppressive is incredibly courageous and really difficult and I didn't have the energy to do it before... But listening to what [the instructor] had to say made me realize how much it's like no it really is important to change every single individual mind... and [if] you can't sway one person's opinion just a little bit, like there really isn't any hope...I don't think I realized how much courage played into it before, I thought it was just knowledge but it's definitely a lot of guts that has to go behind ((laughs))...

Dita suggests that identification with the Girl Power instructor and the feminist movement were important factors in increasing her motivation to want to engage in 
critical action, specifically interruptions. Dita's ideas of changing one person at a time through interruptions, however, may be both grandiose and limiting. They are potentially over-ambitious in that they suggest that every person's mind and actions can be changed through interruptions. This does not take into account the complexity and varied life experiences of individuals. The idea of changing every person also assumes a binary splitting of good and bad, in that the "bad" people need to be changed to go along with the ideals developed in Girl Power. The idea of changing every individual person is also limiting in that it does not address the roots of oppression in institutional and systemic forces that cannot be changed merely through converting individuals thoughts and actions.

Dita also illustrates the connection between hope and the ability to engage in long-term change efforts. Hope is necessary in the face of seemingly overwhelming odds to want to try to change one individual person you must have hope that that effort is part of a larger movement to create change. Hope is also coupled with anxiety, it suggests the need for something different, and thus change may require suffering, and management of that suffering and the disappointments that follow efforts at social change. While hope is often a useful and necessary emotion to avoid burnout, the basing of hope on changing "every single individual mind" is quite daunting. Indeed, participants' belief that interruptions and changing language alone can change the world could be rooted in what Freire (2001) calls magical thinking, a point I return to in the discussion section.

Violet articulates how she bounces back and forth between anger, hopelessness, and hope stating: 
...[I'm] angry that...there's still so much work to be done and I guess there's hopefulness too, because when you look at the big picture in history we have come really far but then it's also hopeless when you think how much further we have to go.

Violet acknowledges that things have improved for many social groups over the past few decades, but she also feels overwhelmed and angry about the amount of changes that still needs to take place. In other words, she is able to deal with this ambivalence, to balance anger at injustices and the possibility for further progressive change. For Violet, a key to dealing with ambivalence is being able to bounce back and forth between conflicting emotions like hope and hopelessness. The ability to acknowledge the progress that has been made while also seeing the need to make further progress opens a potential capacity for long-term commitment to change efforts.

The emotions that both prohibit and enhance SPC consciousness development vary widely. Overcoming fear and developing hope are recognized as important in the process of awakening. However, few participants solely experience positive feelings throughout the entire course. Students more often experience emotions that vacillated. This labile nature of emotions suggests a range of actions or directions potentiated by such fluctuating states. Emotions may facilitate critical consciousness development, but they also may contribute to movement in the opposite direction.

The emotions involved in the process of awakening vary, although excitement is a commonly mentioned emotion. However, some students, such as Bunny, describe the course as "I was emotionally drained" and note that the course can require a lot of selfwork above and beyond what is "assigned." Elizabeth said she was forced to do "a lot of work on myself" and Samantha stated "I had to dig deep and do a lot of self-reflection 
and think about my language..." Participants were able to deal with the draining aspects of the course through relationships in and outside of the course.

Many participants also talk about being anxious or nervous in the course, particularly the rap sessions. Bunny states, "I was nervous, ((softly)) nervous as all hell." She says she was nervous throughout the entire course, but much of her narrative of being nervous centers around the rap sessions. Violet echoes this sentiment "I was pretty nervous doing rap sessions stuff" as did Elizabeth "I was just nervous to work with the actual girls."

The reasons offered by participants for their nervousness around the rap session varied. Some worried that working with the teenagers might trigger memories from their own, painful, adolescent experience. Others were just generally scared of teenagers, and still others worried about being a leader. All of these explanations suggest that the rap sessions brought up feelings of insecurity in the participants. Learning is often an uncomfortable process, as it involves acknowledging areas of previous blindness. However, the level of discomfort experienced can also become problematic if it exceeds students' ability to cope with the discomfort. For some participants Girl Power, at times, exceeded their ability to deal and lead to a need to seek outside resources. At times, the course did not provide enough resources for students to deal with the overwhelming feelings that emerge. During the interviews, Bunny and Violet speak of needing therapy to deal with the emotions triggered during the course and rap sessions. Although the course provides opportunities to debrief and develop relationships to deal with the difficult emotions involved in the processes of awakening and the course in general, these 
resources are not enough for some students. This suggests a potential boundary issue in the course if some students feel forced to over-share or over-engage in the course, although, no participants explicitly stated in the interviews that they felt this force in the course. However, participants did discuss needing therapy and social support to get through the most taxing aspects of the course. Further, students in the course may have engaged in other resistance strategies to avoid engagement in the course such as, not participating in all aspects of the course or the interviews. Finally, a demand characteristic may again be at play in limiting what students choose to share with me during interviews.

The importance of relationality. A reoccurring theme throughout the data centered on relationality. This finding is not surprising given the connection between emotions and relationship. Further, the first research question focused my attention on this aspect of the data: How are emotionally and relationally significant Girl Power experiences related to SPC consciousness development? Feminist and psychoanalytic thinking, both of which stress the importance of emotions and relationships, guide this focus on relationality. Three sites of relationality are identified as central to the process of awakening: the girls in the rap session groups, the instructor, and other classroom participants. Among the classroom participants, the rap session partners are often identified as the most influential and important individual in the Girl Power experience.

Rap session girls. This group consists of the adolescent girls that took part in the rap sessions in the community. I use the term "girls" because this is the term that both the instructor and the participants most often used to refer to rap session participants. 
The rap sessions are not equally powerful for all participants, but many participants do identify the interactions they have with the girls in the community as an important component of the process. For example, Peach states "yeah and it kind of all came together... when we started doing the rap sessions and... just spending time with the girls and hanging out with them... that was really awesome and I felt like it was a safe space that I liked to be in." For Peach, the rap sessions are not just benefiting the girls but also valuable for herself. Like Peach, Remi talks about how she really enjoyed being in the rap sessions, “...I really like hanging out with teenagers, it's cool to meet younger girls and try to... get them to talk about feminist issues and talk about their lives openly cause I never really had a way to do that when I was younger." By using the term "hang out" Remi suggests that the rap sessions were a very informal space for participants where she "tried to get them to talk about feminist issues." This leaves open for interpretation the extent to which the rap sessions moved beyond a space for socializing and into a space for dialogue that could lead to the development of SPC consciousness for the girls and students.

Trying to create a space for teenagers to talk about issues important to them in a nonthreatening and nonjudgmental way seemed to fulfill a need that many participants identified as unsatisfied in their own adolescent experience. On the subject of rap sessions Mint Tea states:

I've been more conscious since I took the course just because, maybe it's from hearing the girls voices and its kinda like hearing myself talk if I had a rap sessions back when I was in high school... and I wish that I did because... in high school no one asked me, 'oh how did you feel about this?' 
Mint Tea talks about the rap sessions as fulfilling a longing she had experienced as an adolescent to have a space to discuss taboo topics. She was also able to connect the girls' voices to her own experience as a teen. It seems that the participation in the rap sessions helps students either work through issues or potentially forces them to relive issues from their adolescent years.

Samantha was also affected by her experience in the rap sessions, she states:

I chose Girl Power... because I wanted to be a part of creating that safe space in the community for these young women to have a voice because at some point in my life someone did that for me but it didn't happen probably at a time that I needed it the most. I didn't learn how to have a voice, when it really mattered... and that's something I'm still working on.

Samantha is motivated to take Girl Power because of her desire to create a "safe space" for adolescents. She feels that by conducting the rap sessions, she is able to connect with her own past self and to continue the process of developing her own voice. Samantha invokes the concept of finding her voice multiple times in her interview, a recurring term in the interviews and idiom in feminist literature and pedagogy. Many interventions carried out in the rap sessions focus on helping women to develop a voice to express themselves in a world that often silences them. One of the directives of Girl Power is to enhance voice in the college and adolescent participants in the project. However, the practice of finding one's voice and giving voice can be critiqued on similar lines to the idea of empowerment (Ellsworth, 1989; Gruber \& Trickett, 1987; Riger, 1993).

The very group structures that gives one group power, thus enabling them to "give" empowerment (or voice) to someone else, can actually undermine empowerment. The structure that gives one group the position to empower others works against the 
process that facilitates empowerment. In order to decrease this problematic position effort must be made to decrease power differentials among group members and divide resource equally, which is often not possible. Further, empowerment is generally understood as an individual level phenomenon that does not account for relationships (Riger, 1993). A group may feel empowerment but this does not always translate into actual political power that can lead to social change (Gruber \& Trickett, 1987). Relating this to Girl Power, participants may feel as though they have an increased voice but this may or may not translate into being able to create meaningful change or maintaining the status quo.

Not all participants describe the rap sessions as a particularly powerful part of the course. In discussing her rap session group Elizabeth says "my group I guess, they were a little hit and miss, so that wasn't a really strong part of the class for me, I think we met with them three times, and it was different girls every time... it was hard to build a relationship with the girls." Since there were different girls present each week, Elizabeth feels the rap sessions lost some of their potential transformational power. However, Elizabeth's rap group partner stated that the sessions were, for her the, most powerful part of the course. Elizabeth's partner, who found the rap sessions highly meaningful, was more similar, demographically, to the girls in the rap session than was Elizabeth. This overlap of life experiences may have facilitated a more meaningful experience for Elizabeth's partner and precluded Elizabeth being able to connect with the girls and thus experience a personally meaningful rap session. Perhaps the difference in life experience brought up levels of anxiety in Elizabeth that prohibited her from being open to the rap 
session experience. Alternately, there may have been a demand characteristic present in the course and/or the interviews. Elizabeth's partner may feel compelled to suggest that the rap sessions were transformational because this is an outcome that was expected in the course. This again suggests the presence of a problematic demand characteristic in the course. This demand potentially limits participants' experiences in the course, prohibiting the full range of feelings and reactions available to them during and following this course. By prohibiting certain feelings and reactions participants may feel stress or mistrust of emotions that feel contrary to the prescribed course experience. Additionally, because these emotions are interpreted as deviant participants may not be able to work through them.

Violet expresses this sentiment, arguing that other aspects of the course were more meaningful to her than the rap sessions. "It was almost like that [the rap sessions] was the least powerful part of the class and I don't want to say it's not valuable, it was certainly valuable, but I expected it to be the most powerful part of the class and it wasn't." Violet is surprised that going into the community was not the most powerful experience for her. It seems that both Elizabeth and Violet seemed disappointed that the rap session experience was not in-and-of itself transformational. This disappointment is not very surprising given that the instructor and course mentors stress the importance and power of the rap sessions and their potential transformational power. This expectancy is potentially problematic in that it prefigures what students are expected to feel and experience in Girl Power. By limiting the range of experiences that can be had in the 
course, Girl Power carries a problematic tendency that is counter to the idea of critical consciousness development.

The instructor. Another important relationship in the class centers on the participants' relationship with the instructor. The instructor was usually viewed as a role model of thinking and living as a feminist, sometimes to the point of being highly idealized. Violet compares herself to the instructor, stating “...I'm not quite [the instructor], [she] is a force to be reckoned with, so I am not quite at the point where I am going to be like she does it, but maybe one day.” Because Violet understands awakening as an ongoing process, she sees the possibility that one day she might be at the stage she perceives the instructor as occupying. The instructor serves as a role model for Violet's development and as a motivation to drive her forward in continuing and applying her awakening.

The instructor is also seen as having a power and passion that the other participants can draw on and use as well as strive for. Bunny puts it this way, "I kept saying to everybody, I want to drink [the instructor]'s Kool Aid, because ((laughs a bit)) [she] was so wonderful." Bunny references the instructor as a figure with the power of a cult leader by referencing the popular phrase "drink the Kool Aid" which is associated with the murder suicide commonly known as the Jonestown Massacre. Interestingly, this phrase also implies a form of blind acceptance of an authority figure without critical thought. However, it is unclear that Bunny was using the phrase in this way. The laughter after the statement suggests that Bunny is using the term in a more ironic fashion, perhaps acknowledging some self-awareness of the potential problems of over- 
idealizing another person. Further, idealization in and of itself is an important part of the developmental process, including the development of SPC consciousness (Haaken, et al., 2012). Yet, the question of when idealization promotes development versus cultivating dependency is important to address. Bunny may be expressing her desire to incorporate the passion for social justice for which the instructor is known. As Elizabeth describes it, "I couldn't get enough, you know, she was giving all this information and she's super passionate and her passion rubs off."

Elizabeth continues to discuss the instructor's passion but she also moves to discuss her form of teaching:

I mean, her passion is very contagious and you can tell she's extremely knowledgeable and very real and approachable and I think that makes a huge part of it. Like she said so many times 'I'm not gonna stand here and teach at you guys', she's learning from us and she's just facilitating it, so yeah I think the way she approached the class with this kind of equality mentality was huge.

Elizabeth also discusses the instructor's use of feminist pedagogical methods in the course. Participants in many of the interviews discussed the fact that the instructor functions less like a traditional authority figure and more like a friend and/or facilitator. Mint Tea comments, "I felt like the role she played was our facilitator and friend rather than our professor." She acknowledges that the relationship with the instructor in Girl Power is different from other courses in that Girl Power was a more egalitarian ethos compared to other courses. However, she struggles to find the correct terminology to categorize the instructor's role in the class. This uncertain terrain is not without potential problems. Elizabeth goes on to state “...she approached it as our equal but clearly had far superior knowledge on that topic, but she was able to share that in not a superior 
function so I think toward the end, because we actually had to do something... it got a little jumbled." In this quote, Elizabeth acknowledges several issues. First, even in classrooms that strive for a more participatory environment with a less hierarchical relationship between teacher and students, the playing field is not totally equal. The teacher still has more power, control, and knowledge. Another tension involves the conflict between the laidback atmosphere of the class and the strict deadlines and rules in place for the completion of the Zine project. Elizabeth describes how some students had trouble navigating the relatively informal and communal nature of the class and the strict requirements to finish the Zine on time-a source of tension taken up in the Discussion section.

Dita discusses how the instructor approaches her efforts at social change:

Just being able to see how she [the instructor] approaches life and how it's zero percent aggressive... seeing how she interacts with the world was just inspiring to me to want to be more like that because she's not a threatening presence, scary feminist person... she's just, dealing with the world with a much more appropriate response and I realize that I wanted to be more like that because she's so likable...

According to Dita, the instructor serves as a role model. The instructor is seen as particularly inspiring in her capacity to model an approach to social change work that is not rooted in what students perceive as aggression. Other participants suggest that the instructor counters the idea that being a feminist (and fighting for social justice) has to be angry and threatening as suggested by the derogatory term "feminazi."

Many of the participants see the instructor as a role model that fights for social justice but does so in a way that still allows room for more traditional expressions of femininity. One participant commented on how the instructor is "really pretty" and 
another commented on the instructor's gender presentation as highly feminine. Both were considered attributes that help make feminism less threatening and scary. While the instructor is able to interest a wider swath of young adults into feminist and social justice movements and insight them with passion for social change work, it is possible that participants may regularly judge other members of the feminist movement that do not present or work for social justice in this way. Participants also may feel limited if they are unable to negotiate the tensions of a feminist identity in the way demonstrated by the instructor.

While relying on anger as the primary fuel for justice work is limiting over time, anger can be a good motivator for short-term actions. The fear of "angry feminist" seems to register anxiety that participants experience around maintaining relational ties threatened by this awakening consciousness. Participants struggle to simultaneously analyze problematic aspects of traditional femininity (e.g., interpersonal aggression) while maintaining identifications with traditional femininity (e.g., wearing makeup). While traditional femininity places limitations on women, it also can serve as a sight of pleasure. As participants learn to critique some aspects of traditional femininity, they struggle with how to deal with practices from which they still draw pleasure. Participants experience ambivalence as they struggle to find new boundaries for femininity and feminism in their life.

Other class members. One last important relationship in the course is that among classmates. Violet states "it was so useful to talk to people about their own personal experiences." Violet suggests that being exposed to and in dialogue with others was a 
major motivator in her changing perspective. Hearing other stories not only gave Violet a new way of viewing other participants but also herself. Violet stresses that her growing knowledge develops not just from exposure but specifically friendship with others.

The class is structured so that community is, ideally, built with all participants and through multiple chances for bonding and debriefing. When discussing the class Mint Tea stated:

...it really is a great way to learn where you feel there's no one superior to you and you're sitting in a circle and you just feel... I mean, I'm used to lecture courses where there's a 100 other people, I don't know everyone's names, I don't have a relationship with them... but in that [Girl Power] course I felt we were a family, we were all connected we all had relationships it was easy to ask people for favors... it was really comfortable and warming.

For Mint Tea the course was successful in creating a community structure that promoted sharing and relationship building. She uses the metaphor of family in suggesting that the connections in the course were not without conflict. Families have some level of inevitable conflict. So by choosing this term Mint Tea may have been suggesting that relationships in the course were more complicated than they appeared. However, it does seem that for the most part Mint Tea experienced a positive environment that facilitated comfort and sharing. In that the idea of community was often stressed in the course, Mint Tea may also be responding, however, to another demand characteristic of the class.

Elizabeth also talks about how the style of the course differed from her previous experiences in the classroom, "I remember going into the class, I hadn't ever talked to anybody else in any of my other classes, and I was like wow this is awesome, I know other people's names..." Elizabeth was responding to a question I asked regarding what from the course she found most memorable. She was one of many participants that 
responded that the structure of the class that allowed relationships to develop was one of the most impactful parts for her. For many participants the communal nature of the class was set early in the term with the social identity circle commonly named as a powerful moment in the class that cemented the relationships in the course. That said, classroom community in and of itself does not lead to critical consciousness development.

Classroom community creates the groundwork for working toward critical consciousness (e.g., dialogue).

The identity circle consists of the entire class standing in a circle and making statements about their past experiences. The statements started out lighthearted (e.g., I was a cheerleader) but quickly became more intense (e.g., I was raped). When a person made a statement, they would step into the center of the circle and other members of the class could step in to say they had also had that experience or students could step in the circle in solidarity. For some participants this experience was one of being surprised by how many people had experienced trauma in their past and for others the activity let them know that they were not alone in their experiences, thus building the classroom community. Sparkles also mentions the identity circle as an important class component: Yeah. Identity circle, I was very surprised that day and I was happy we did that activity, cause I remember I had been going through a lot of stuff that whole week ... everybody just coming together and really being honest about things that they had gone through and being open just helped me be like, oh, this is an awesome group of people, I remember I cried that day, in class, but it was awesome, I really like that activity.

This activity seemed to serve the dual purpose of bringing the class closer together and normalizing student experiences that are often kept secret. For example, when a woman is able to see and hear that other women have been sexually assaulted, she can begin the 
process of making that personal experience into political understanding of how sexism, patriarchy, and power function in the world. Further, sharing these events with others may help free students of any shame they may have felt around the experience. Despite the perceived usefulness of this activity, participants suggest it can also be emotionally triggering. I take up this potentially problematic aspect of the identity circle in a later section.

Participants tend to develop the closest relationships with their rap session partners; they provided encouragement when individuals became overwhelmed and frustrated and provided support surrounding the rap sessions. Bunny discusses her rap session partner this way “... she was my other person in this. Like her and I just glued together and her and I were just like okay let's do this." Samantha shares a similar sentiment:

It was, __ _... she made it enjoyable for me, because I had someone on those day that I just didn't want to be there, I was just F-this, she was like 'no we can do this, lets do it together', and so having a buddy in the class that can feel your pain and was experiencing some of those same emotions was great.

Samantha emphasizes the importance of having a person with whom she could work through negative emotions. In this quote, Samantha also suggests that there were days when she did not want to be in the class and struggled to show up. This suggests that at times she felt triggered by the course and pushed beyond her available resources. However, having one person to encourage her through these times provided some motivation to get through the difficult aspects of the process. This quote suggests that the relationships in the course are linked to emotion in the processes of "awakening."-and in staying awake. 
Elizabeth also talks about the importance of her relationship with her rap session partner “... a big part for me was my partnership with the paring made a big

difference for me... having somebody that I knew I could rely on...” The individual pairings provided an additional resource for students to debrief and discuss the emotions and thoughts brought up in both the course and in rap sessions. This additional level of relationship seemed to help participants' navigate the emotional terrain of the rap sessions.

Participants not only discussed changes in thought because of their processes of awakening but also change in their actions. The next section presents findings related to how the processes of awakening were applied to participants' behaviors in different areas of their lives.

Application of awakening. Critical consciousness requires not only the development of critical reflection but also engagement in critical action. Understanding what moves individuals from critical reflection to critical action was one of the guiding questions of this dissertation and motivated the focus on emotions and relationships. Students applied their processes of awakening primarily through the course concept of interrupting oppression, with participants being particularly vigilant to interrupting hurtful language.

Participants overwhelmingly reported that interruptions were the primary way that they carried their processes of awakening outside the classroom and applied critical consciousness in their own lives. Participants not only stated the importance of interruptions but many had instances of utilizing this practice. Bunny gives an example: 
What I really took away from Girl Power was interruptions. Like I interrupted my mom one time because she called my childhood friend a whore and... I like totally felt [the instructor] like come into my body and just say, 'now why would we call her a whore? How does that help the situation at all, why are we classifying her as that?' It really made my mom think, and now she doesn't use the word whore anymore.

In this example Bunny interrupts through questioning her mother's use of the term whore.

Bunny attributes the power of this intervention to the instructor. This attribution takes away her personal initiative in the process and suggests a quasi-religious experience of being possessed by the spirit of another person. This attribution could suggest that Bunny may not have integrated the course values into her sense of self. Alternatively, Bunny may also be using the instructor as an orienting or grounding object in her processes of transformation as she struggles to gain her own footing in her processes of awakening.

Mint Tea provides an example of using interruptions on her friends:

My girl friends will be like 'oh do you see what that girl's wearing?, she looks like such a slut' and I'm like oh you know, you shouldn't call peoples sluts... but what does it really mean? Like who gets to decide what's appropriate and what's not?... what is the meaning behind that word?... think about it, how would you feel if like other people were calling you a slut?... who really gets to define how a woman dresses?... So I've been interrupting my friends, I've been practicing on them because it's a little bit easier than strangers but I feel like I'm passing along the message to them.

Like Bunny, Mint Tea also uses a process of questioning to interrupt her friends use of the term slut. This style of interruptions attempts to avoid saying, "you are a bad person for using this word," and instead locates the negative connotation of the word in a broader system of oppression of women. Initially her friends are confused by Mint Tea's interruption, but later in the interview she states that "when they talk to me they are a 
little bit more cautions of what they say" because they have begun to expect her to interrupt. Mint Tea and Bunny's examples are representative in that they show participants interrupting friends and family members. Mint Tea expands on this dynamic by saying that it is easier to interrupt those that are close to you as opposed to strangers.

Mint Tea also uses another tactic in interrupting her friends' language:

I took a Women's Studies course before and I kinda had like a vague understanding of feminism... and then when I took [her] course and being able to, I feel, like actually apply feministic work... now I actually pay attention to what people say in their conversations and little things where before I would just let slip by but now I'm like, oh you know that's kind of oppressive or that's kind of hurtful and that kind of makes me feel this way and I feel it probably hurts other women to and maybe you shouldn't say things like that... [emphasis added]

In this quote, Mint Tea demonstrates another strategy for interrupting, she uses feeling language and qualifiers to soften the interruption, making the interruption seem less invasive and aggressive. Mint Tea also suggests that Girl Power succeeded where other courses had not in that it offered a concrete way to apply newfound knowledge.

For some participants they were drawn to the Girl Power model of creating change because it was seen as less aggressive and conflict oriented as compared to some other models of critical action such as protest. Sparkles states, "I feel... your creating change in a way that isn't like going out there and like getting in fights with people, it's like your talking about a topic that people don't normally want to talk about." The interrupting model of oppression felt more accessible and acceptable to Sparkles because it was based in talking and perceived as less aggressive. Elizabeth also prefers the method of interruptions because it is viewed as less confrontational:

Because it's one thing to be like here is what's going on, here's how it is in everyday life and how people are using oppressive language and you should stop 
it. But it's another to be like, all right, that's not gonna get you very far this is redirecting, educating, things like that made a big difference cause it's less confrontational.

Elizabeth notes that interrupting in and of itself can seem confrontational. The act of interrupting someone when they are speaking is seen as rude in many groups. Further, telling someone that they are being oppressive and should change their behavior is also potentially discourteous and may be perceived as highly confrontational. However, Elizabeth is able to deal with this dissonance by suggesting that interruptions do not change behavior this way and instead they "redirect" and educate, highlighting two variations of interrupting.

Few participants, at the point of the interviews, had moved to interrupting people outside of their immediate social circles, although Violet is an exception to this. Violet recounts an experience of interrupting oppression:

I had no idea that the term gyp, was related to gypsies and so then I think I heard somebody that I knew say that and I was like, ((takes on a lecturing tone)) did you know that that's offensive to ((laughs)) gypsies or something to that effect, like when you say that, what that really means is, and they were like I had no idea either so don't feel bad, neither did I ((laughs)).

Violet states during her interview that she likes to approach interruptions from a place of humor. She does that here by taking on a jokingly lecturing tone when interrupting but then quickly switches to laughter to try to keep things lighthearted. In addition, she tries to avoid making the person feel bad for using the word by stating that she also just learned that the term was offensive, so she tells the person, "don't feel bad." This response to interruptions may be because of Violet's previous experience with the practice. It seems that before Girl Power, Violet had a practice of trying to interrupt 
oppression but only with those close to her and often the results were perceived as negative, "went over their head" or "started a fight." For Violet learning about interruptions in Girl Power gave her not only the confidence to interrupt those outside of her immediate social circle but also the skills to do so in a way that minimized interpersonal conflict.

Many participants talked about how the practice of interruptions changed them in a broader sense. For example Peach states, "Learning and practicing about interruptions really changed me in a major way, I do an interruption everyday now, like [the instructor] said she does and I do too and it feels really good and really important to fight against oppression." For Peach, interruptions have become a daily part of her life. This integration of the practice into her life seems to have been facilitated by the fact that engaging in interruptions offers some positive ego recognition. For Sparkles, the course helped her overcome her fear of interrupting:

Taking that class really helped me learn how to do interruptions which was really helpful and I feel like after that class I've noticed the things that I say and the words that I use are completely different than before taking it... because I was always scared like oh this person's gonna freak out or I don't know what to say. Sparkle's quote represents a common tendency of research participants. Many speak to the fact that before the course they saw oppression and heard oppressive language but did not intervene, either because they were scared or because they felt they did not know how to intervene. Participants also note that the course has sensitized them to instances of oppressive language. It seems that the course's ability to help students overcome fear and learn a concrete and seemingly applicable way of dealing with oppression were 
major factors contributing to their ability to carry the process of awakening into action. I now turn to address the second theme, sources of dissonance.

\section{Listening to Girl Power: Sources of Dissonance}

Participants also describe the processes of awakening as fraught with conflict and complications. These difficulties arise when participants' newfound view of the world is met with resistance from both internal psychological forces and outside social forces, including friends, partners, and family members. These sources of dissonance both drive and inhibit participants' movement towards higher levels of SPC consciousness. In this section of the findings I move to address the second research question: What tensions arise between the dominant culture and/or significant others' values and the values of the Girl Power capstone? The second part of this question, How do these tensions move individuals toward or away from critical consciousness, is addressed more fully in the discussion section.

Although the primary sources of dissonance vary, all of the participants express some level of conflict in developing SPC consciousness. Some of this dissonance deals with the internal and psychological forces (dissonance inside) while other aspects deal with the external and social forces (dissonance outside). The role of Girl Power in dissonance varies within and between participants. The Girl Power experience, whether in the course or the rap sessions, is an important site of felt dissonance. At other times the knowledge and skills gained from Girl Power indirectly causes conflict in other aspects of participants' lives. The most commonly cited and most problematic source of dissonance centers on interpersonal relationships outside the class. Students talk about 
Girl Power impacting their friendships, partnerships, and family relations. These different areas of dissonance make up the subthemes for this chapter and are the focus of the rest of this section.

Dissonance within the self. Many participants talk about experiencing internal conflict, for some this takes the form of conflict among emotions. At other times, struggle between cognitions and on occasion a clash between internal thoughts and behaviors. Violet talks about dealing with oppressive language: “...the things that I've been programmed to say off the cuff that are possibly offensive to people, that in my heart of hearts I would never want to hurt anybody, but it's just the social conditioning." Violet struggles with the knowledge that she has gained through the course and the difficulty of breaking habits of saying certain words. She seems horrified by her capacity to perpetuate oppression through the use of learned language. Violet deals with the dissonance of being hurtful but seeing herself as a "good" person and placing the blame on society- "its just social conditioning." By placing the blame outside of herself she is able to overcome cognitive dissonance. However, by externalizing the blame she risks a loss of motivation to change personally because she places all of the bad on the outside. This is problematic in that this external environment becomes a part of one's conscious and unconscious landscape as they grow up in a culture that perpetuates oppression. In order to address oppression, which exists at multiple levels (Young, 1990), it must be attacked on each of these levels, including the psychological.

Remi also discusses dissonance around language, but for her this dissonance is a driving force for development. I asked Remi "if you were to think back at what point 
over the past few years did you have your biggest increase in that [feminist consciousness] where you started to see things in a new way?" She responded, "Probably when I did things, or said things that were kind of ignorant and then I thought about it and realized that that's weird and I shouldn't do that or think that way. And I still have those moments." Remi suggests it was the times when she felt a disconnect between what she was doing or saying and new ideals that prompted further development. She indicates that the feeling of dissonance was a signal that something was wrong. However, she also acknowledges that feminist consciousness is a process of development and that she still has these moments of "disconnect." Samantha also seems able to deal with the dissonance in herself and in others by reminding her that development is a process, and a hard one at that. By telling herself it is a process, she seems better able to deal with the ambivalence that arises when dissonance is encountered:

I find myself more and more doing interruptions and interrupting myself and really thinking about my language, because language is powerful. So... just being okay with knowing that this is a process, it's the language that we speak we didn't learn it overnight and so to deprogram and reprocess that it's okay if you make those mistakes sometime.

Dita talks about the difficulty in becoming "hyperaware" of the injustice surrounding her:

... there was definitely a moment in the middle [of the class] where I felt so hyperaware outside of class that it almost made me feel really uncomfortable. I was all of a sudden like, 'maybe I don't want to be so aware' and immediately realized, no, no, no you really do and it's better this way because that's how you move forward instead of just feeling trumped by these situation's. There's definitely a point in between where we were still in the process of learning where I was like 'if I left the class right now I would just be super aware of how unfortunate everything is and I wouldn't be able to do anything about it.' 
Dita acknowledges that her process of awakening causes discomfort, in part because oppressive situations are now more salient. She seems hesitant to acknowledge this struggle, however, in adding that, "it almost made me feel really uncomfortable." This reluctance may result from the demands of the course or the interview that suggests that the process of awakening is a positive experience. This simplified interpretation of the experience diminishes the emotional turmoil that is expected in any transformation. She seems able to overcome some of the uneasiness by learning specific behaviors for expressing or applying her process of awakening. She expresses a certain depressive burden of consciousness, of not being able to unlearn what she has discovered. I return to this idea of wanting to unsee what is now seen in the discussion section.

When I ask Elizabeth directly if she experienced any tension or dissonance when taking the course, she responded:

I mean, maybe with some of the things that she [the instructor] was presenting, like I'd get upset because that's something that I had been doing. Like I had caused more oppression I guess, so maybe like just frustration with myself... and just more like dissatisfaction of wanting to do more.

For Elizabeth, dissonance emerges in the realization that she had been engaging in oppression herself. This dissonance causes her a sense of frustration, which in turn motivates her to want to engage in more action, suggesting that frustration at one's self has both limiting and expanding capacities.

Dita suggests that she was able to "jump past" the frustration involved in the process of development, "...I feel like I got to jump from being oblivious to being very well informed and that I didn't have a middle period of just feeling utterly frustrated but not having any way to express myself..." Dita suggests that she is thankful that she did 
not have to deal with the frustration she assumes would come in the middle of her development; she feels that she was able to "jump past" the turmoil of the middle, transformational, level of development.

Violet talks about the importance of a safe space in dealing with dissonance, "I mean you have to create a safe space for people to say what they feel, to think what they feel, because I think a lot of people struggle with allowing themselves just to think about something without beating themselves up." The thoughts and feelings with development can seem deviant because they counter a person's previous ways of knowing. One of the important aspects of Girl Power was allowing space for participants to intellectually explore both academic material and personal experiences and how they may intersect in an open and safe communal setting. Violet seems to suggest that Girl Power played an important role in helping students transfer emotions and thoughts that previously felt deviant because they counter dominant ways of knowing.

If the identity matrix of Violet felt safe in Girl Power, it is possible that members of other identities did not feel so safe in this same space. Bunny states, for example, "I hated being a teenager... I don't want to go back to that, and so like, these girls are in it, and I'm like I feel terrible for you." For Bunny and other participants, rap sessions brought up feelings of their own difficult adolescent experiences. Some participants hoped that returning to this difficult period would help them work through some underlying tensions. Other participants expressed that being vulnerable opened the door to further development. Samantha discusses her experience of feeling vulnerable in the 
classroom, particularly around her marginalized identities and re-experiencing difficult aspects of her earlier life:

For me it's difficult sometime being the only sitting in the classroom, because, usually when we talk about what's happening in the world we're talking about it from a dominant perspective, well that's not me and so, to find a way in which I fit into all of this, it's not always easy. There were times when I've gotten upset... but it also makes you have to do a lot of personal work... and when we think about childhood and things that you've experienced that it didn't bring up some feelings that you probably wanted to bury forever

Samantha simultaneously recognizes the difficulty and necessity of integrating and understanding past experiences into her processes of awakening. Further, the difficult aspects of the course led her to a place of coming from love and striving to continue to grow and be "a better person." However, she also acknowledges that the course was difficult at times, which was a common theme in the interviews. These participants with negative adolescent experiences seemed to view the course, particularly the rap sessions, as a form of therapy for dealing with their past pain. I return to participants' association of the course with therapy in the discussion chapter.

Dissonance with the Course. A second area of tension identified by participants was felt within the course. Participants talk about various frustrations and conflict they felt in the course throughout the term. Sparkles talks about the day the group engages in participatory decision making to pick the title of the Zine:

I remember that day being frustrating cause everyone was coming up with ideas and then other people weren't coming up with ideas and then the people that weren't coming up with ideas were like 'oh I don't like this, I don't like that' but they didn't have anything better.

Sparkles feels that other members of the class were quick to offer criticisms but not to propose helpful suggestions in picking the Zine title. Elizabeth is also frustrated with the 
process, "Picking the title not a fun process ((laughs)), I was not a fan ((laughs))...I guess what I liked about the class in the beginning, about it being open and everybody talking kind of backfired, towards the end and it got a little old..." Elizabeth suggests that her sense of anger was coming from something she had previously thought was a benefit of the class, allowing room for everyone's voices. Elizabeth goes on to discuss her frustration she felt towards the end of the course:

We had a Zine making day and I think... we needed a little bit more direction, a little bit more time management. Because I value my time... And towards the end there we were just like, did we do anything today? Other than talk about, the weather?... I think there was a feeling of frustration on some ends, by the end, especially when we were going through the [themes] list and like lets get through this, c'mon.

Elizabeth suggests that other members of the course shared her feelings of frustration. For her the sharing and community building was a strength in the beginning of the course. Nevertheless, as assignments became due, the participatory decision-making of the course no longer seemed beneficial and was rather seen as inefficient. Towards the beginning of the course the activities designed to increase community building may have been perceived as more fun, with no real task attached to the activity, outside of building community and critical reflection. However, once the class process demanded that certain task be met, Elizabeth began to feel anxiety about these tasks getting completed and what was previously perceived as enjoyable quickly began to feel like a waste of time.

Frustration with these strategies is common. Individuals, particularly in the education system, are used to being told what to do; authoritarian decision-making processes are quick and relatively easy. This is the type of decision-making that is most 
often deployed in the classroom, the teacher makes the decisions and the students follow suit. A key component of Critical Pedagogy is offering the students power in making decisions. However, some students may not want this power, they may still feel more comfortable being told what to do. Further, some students do not seem to value the process itself so they are not willing to go along with the extra time and effort that is required to make a decisions when everyone's voice is heard, particularly when the process is no longer perceived as enjoyable or becomes imbued with anxiety because of looming deadlines. While participatory decision-making is not in an of itself a component of SPC consciousness, it often works as a driver of development towards critical consciousness.

It was common for participants to express frustration with the course process at one time or another. While Sparkles and Elizabeth were aggravated at the end of the course, some participants were irritated towards the beginning of the course, Mint Tea states:

At the beginning I didn't really know what she [the instructor] was talking about because we didn't really do any lecture on it and the class is kinda just more like, 'oh we're getting to know each other'... like I didn't really understand why we had all these check-ins that some of them would last like half an hour which made sense and then others would last for like a whole hour... I was just so used to lecture based classes that I was just like kinda waiting for that material to come and then I realized that the most important part of the class was getting to know each other.

Mint Tea had trouble adapting to this style of course because it was so different from her previous courses. Mint Tea's struggle to understand the course time that was spent on process (e.g., forming community) as opposed to learning content. She continues: 
I felt like I was bouncing back and forth between being okay, I'm vulnerable, I'm kinda overwhelmed, to oh I'm excited, and some things were kind of stressful, and so I felt like my emotions were just like bouncing all over the place. Towards the end for the most part I had like a really positive feeling about everything.

Mint Tea alludes to the labile nature of her emotions. For her, Girl Power elicits a broad range of changing emotions. However, she states that towards the end of the course she began to have a more steady and positive opinion of the course. Mint Tea goes on to say she talked with other students who shared this view of being overwhelmed and lacking structure in the beginning. For students that felt some frustration with the course, whether in the beginning or the end, it seemed important for these participants to either seek out confirmation that others were having a similar experience or at least justify their position by telling me they were not alone in their feelings.

For some students, particular activities elicit feelings of dissonance. The identity circle is described as a particularly emotionally demanding course component, Violet states:

The identity circle that was very difficult, it was especially difficult because she [the instructor] had said even if you hadn't gone through this maybe you should step in the circle so someone doesn't feel alone and nobody stepped in the circle for me when I said something and then I was in my car later crying. And then I was like, what the hell? Why am I crying about this? But then I realized you never know how you truly feel about something until you uncover those layers.

The activity was upsetting to Violet because she feels no one supported her during an activity, which is supposed to be about solidarity. Violet's experience in this quote and at other points in her interview, suggest that she did not develop many strong relationships in the course, as many other participants suggest they did. Violet was able to experience some personal transformation in the course without these peer relationships, although she 
does note that she felt a bond with the instructor. Despite the difficulty of this aspect of the course she puts a spin on it in suggesting that this was another area where she was able to work through feelings and grow in the course. This may reflect her interpretation of the experience or it may reflect demand characteristics of the course or interview that suggests the need to paint Girl Power as a positive experience. Finally, at other points in the interview Violet notes that she had to use resources outside the class, such as her job and therapy, to deal with the difficult experiences and feelings that arose in the course.

Bunny struggled in the identity circle and also had to draw on outside resources to deal with the experience, saying:

I've had to go through with therapy and stuff so like when we were talking about like social mapping, I was like hell no, identity circle, that was so tolling... It was like, yes this was unifying, but it was also taking a toll on my psychological well being. Luckily I still see a therapist regularly so I got to be like I'm tired, I'm drained, help.

Unlike Violet, Bunny felt a great deal of solidarity during the identity circle and she felt that the activity helped to cement relationships she was having in the class. However, she describes the experience as taking a significant emotional toll, so much so that she had to discuss the experience and get "help" from a therapist.

For many participants conflict in the classroom is perceived negatively. This is the case even when the conflict was a part of the participatory decision making process. Mint Tea was surprised when the structure of the course led to disagreement:

I was surprised that people didn't agree and there was just so much back and forth going on, but I don't know, maybe they were just very opinionated and they were advocating for themselves I guess but it was just really hard for us to like find common ground on a lot of topics. 
For Mint Tea, and other participants, the characteristics of a healthy community seems to include always getting along and agreeing, in other words the absence of dissonance. When discord does occur this does not match their view of the class, potentially limiting the ability of students to bring up feelings and thoughts they had that were seen as dissonant from the goals of the course. I return to this fear of conflict in class in the discussion section.

In addition to experiencing dissonance within the course, many participants experience some level of dissonance while conducting rap sessions. Remi says that the rap sessions were "challenging because it forced me to be outside my comfort zone." Peach similarly states, "the rap sessions pushed me out of my comfort zone.” Both women talk about struggling with the rap sessions because they put them in a situation they have never experienced before. However, Remi and Peach had self identified powerful experiences in their rap sessions that led them to either continue or desire to continue to work with teenagers in a similar capacity.

Dita found the rap sessions particularly anxiety provoking:

So I was really nervous at first and that was because I knew there was going to be the whole teen girl component... I felt like a total fish out of water like I'm not going to know how to relate to these girls even a little bit, which I couldn't be more wrong. Because I completely related to them and... the girls I got fortunately were very close to the kind of upbringing that I had had... so I connected with them much more than I thought I would.

Dita talks throughout her interview of her trepidation regarding teenage girls and the feelings that the entire course brought up for her regarding her difficult growing up experience. She was able to work through these feelings and have a self identified powerful experience in the course and the rap sessions that seems to have helped her not 
only develop critical reflection skills but also develop more meaningful relationships in her life. The rap sessions not only produced dissonance as participants struggled with their own issues, but also how they helped the girls work through issues related to adolescence. Dita continues:

Keeping positivity in our rap sessions was a really difficult thing, I can see just... how intensely women are really pitted against each other and placed in the positions of competition was very obvious when we were there cause it was a very loving group... but there was definitely a lot of cattiness also between the girls and it was very hard to defuse without making them not feel like we were attacking them. That we're not, not saying you're a bad person but your using the wrong language for this... that was the hardest part to approach was to explain how detrimental it is to them to constantly be judging each other.

The rap sessions not only illustrated a point of critical awareness for Dita, they also gave her practice in taking action on her awareness. However, the process was not simple. She acknowledges that she struggled with how to interrupt the girls without placing blame on them. By using the term "wrong language", Dita seems to suggest that there is one correct way of saying something, which may indicate a somewhat limited or closed view of the world. The idea that there was right and wrong language was common throughout the interviews. While I understand that participant's are working to counter oppressive language, the idea that there is a prescribed way of going about this seems to involve a form of psychological splitting—of separating the world into all good and bad categories. This all-or-nothing mode of thinking prohibits more complex and nuanced ways of understanding the world.

Dissonance with recreational activities and culture. Many participants mentioned a changed relationship with popular culture and other activities used for 
enjoyment and relaxation following their Girl Power experience. Mint Tea talks about her shifting understanding of advertising specifically:

Oh my gosh when I watch TV and I see commercials I'm like everything is wrong with that commercial. Especially when I see the girls in bikinis eating the hamburgers I'm like this is portraying so many negative message... before I was like 'oh yeah that's funny' but now its just, 'oh my gosh that is so wrong and that makes women look bad and I'm a woman and I would not want women to think that way of me'...

Mint Tea suggests that Girl Power helped her to be better able to interpret the messages presented in the media. This changing orientation has taken some of the fun and humor out of previously enjoyed activities. Remi shares a similar experience of developing new views of popular culture specifically:

Just being able to analyze the messages that media sends to people, especially women and girls... I mean its totally changed since I first started learning about feminism. Also, like the music that I like or that I used to like a lot, I started realizing it was really like male centered, like all dude bands and stuff and singing about dude problems I just kinda got bored with that so I stared listening to more music by women.

As her outlook on the world changes, Remi's previous choice of music is no longer interesting or fulfilling. For many participants this change was marked by feelings of dissonance. When I asked Peach if her reaction to recreational activities had changed, she responds "yeah, it sucks." I asked her why "it sucks":

I mean its totally positive, but there's TV shows and there's music that I like on some levels that I now have trouble listening to because... it's oppressive on one level or another and its just all up in your face all the time. [Back] when I was saying 'you can't take it out, you can't take it back,' not that you would want to but... [for example] I like to go out and dance... and that's where a lot of these negative stereotypes are being propagated is in like mainstream music and videos. I've always taken issue with offensive... music on some level and music videos as well, but it's maximized to a point where it's more intolerable to me now where before I could just be like, 'whatever I know that that's offensive and not okay but I still really like this song'... 
Peach struggles with her changing relationships with media as well. Her new critical lens has made it hard to enjoy experiences that previously brought her pleasure and/or relaxation. She does not credit Girl Power with being the sole source of seeing and hearing oppression in media, but rather points to the course for making these oppressive aspects seem more intolerable. A part of Peach seems to wish that she did not have this new added sense of injustice. She references an earlier part of the interview where she says, once you have the knowledge you cannot go back to seeing things as you previously did, no matter how inconvenient or uncomfortable it may be. Peach, like other participants, struggles to find a balance between enjoyment and critique of culture involved in recreational activities, like dancing, which creates some level of anxiety and potentially resentment towards Girl Power in creating this new area of tension.

Violet also discusses her changing relationships to fashion:

If I look at a Vogue magazine, I enjoy the fashion and I enjoy the stories and so on and so forth but then you sort of, struggle with that feeling of, am I being shallow? Or am I being superficial? Or should this even matter?... to have that balance its okay to take care of yourself and to improve your appearance but its not who you are.

Violet is able to acknowledge that she enjoys some things that might be seen as potentially oppressive but she also interrogates this feeling by asking, "where does this come from?" She seems able to deal with the dissonance of knowing something is potentially oppressive but also getting enjoyment out of that same thing. She seems able to handle this area of ambivalence without completely pushing away previously enjoyed things. However, this quote could also be interpreted as Violet being lost and confused 
regarding the dissonance she feels post Girl Power. Violet continues to discuss her relationship with fashion, beauty, and gender:

But yeah, I mean, I still struggle with that because... I struggle with it in the fact of gender identification being a woman the things that I enjoy, do I enjoy them because I was sort of raised in that culture? Or do I enjoy them because I'm a woman?

Again Violet probes her feelings and attitudes, where does her enjoyment from? Is it an aspect of culture or is a deeper feminine connection? Following this statement during the interview I asked how she dealt with this struggle and she responded, "I just try to find a balance... Maybe I don't deal with it very well, I don't have an answer I wish somebody would say, here's the answer." Again Violet returns to the idea of trying to find balance between being critical but also still allowing herself enjoyment, an important developmental capacity. However, she also questions herself and her solution- she would like someone else to give her a better solution. The new lens that she developed in Girl Power has complicated her life and she struggles to make sense of her new relationship with the media. She may still feel like finding balance is not a perfect solution because she still feels dissonance in her relationship with culture as she questions, why do I feel this way? Should I feel this way? This continued level of dissonance is also potentially limiting and harmful. Without a community of others to discuss this tension with, she may struggle with her dissonance and choose to resolve the tension by no longer being critical of media or no longer being able to find enjoyment from media. Few participants seem to have a community post Girl Power that is able to provide them with their needs as they struggle through the dissonance of changing awareness and actions, a point I return to in the discussion chapter. 
Dissonance and Relationships. Another sources of tension for participants was found in their relationships. Participants seemed to particularly struggle with this source of discord. It seems that outer sources of dissonance, such as with recreational activities and culture, could add to the dissonance felt in relationships as individuals involved in Girl Power change in ways that are not reflected in their surrounding community. In this section, I address dissonance in regards to participants' friendships, relationships with men, and their families.

Dissonance with friendships. Many participants discussed feelings of dissonance in old and new friendships following Girl Power. Remi articulates the issues, “...like having people in my life I guess who don't know what I'm learning, it's like not part of their consciousness so it's kind of hard sometime to be around [them]..." Remi acknowledges that what she has learned has changed her and that this change has resulted in tension with those that do not see the world as she now does. Violet discusses how her process of awakening disrupted her relationships with an older group of friends:

I have friends and... it's just not as fun being around them anymore, I just feel like they're not as with it. Like they're sort of stuck still in high school. I don't know if that has to do with Girl Power, maybe its does, maybe I'm becoming more of a feminist or ((laughs a bit)) something I don't know, it's just hard to go back to where you grew up and see, especially your female friends and, just putting up with $\mathrm{x}, \mathrm{y}, \mathrm{z}$ and not sort of being proactive and I feel like that does boil down to social justice, like they've drank the Kool-Aid or something and its not because I feel like better than them...

Violet feels that she has progressed but that her friends have not, although she is not sure what exactly has caused this personal transformation. Violet seems disappointed in this group of friends and accuses them of having drunk "the Kool-Aid." She uses this phrase in a different way than Bunny (discussed in previous sections) in that Violet accuses 
those who have not gone through a process of awakening as having drank the Kool Aid, whereas Bunny suggests that she drank the Kool Aid in her process of awakening in Girl Power. For Violet "drinking the Kool Aid" is associated with regressive tendencies of people in her past that she would rather not be associated with. For Bunny, this phrase represents an aspect of her progressive transformation. This cultural idiom takes on opposite meanings for these two participants experiences.

Peach also discusses tension with her friendship group. After I ask Peach if her group of friends or community has changed post Girl Power she responds:

There are people I don't hang out with anymore based on our different philosophies... The problem I'm having is that I have a couple of good friends who don't want to be oppressive... [but] I have a harder time... interrupting what they're saying and getting a positive result... It's kind of sad, you feel kind of lonely, in that now I'm thinking about these things and I wanna share it with these people but maybe they don't want them? Or they don't wanna be in community with me about these things. So then I kind of just feel alone and not really safe with these people because they've already kind of damaged our bond by saying something that I think is oppressive.

Peach discusses dissonance in two areas of friendship. First, she talks about tension in relationships with people in her broad friendship circle. To deal with this dissonance she says she stopped hanging out with these people. She then discuses the dissonance that is developing with two close friends and the pain and anxiety that this changing relationship produces. She struggles to interrupt these friends. The result is that Peach feels sad about losing connection with these friends and describes how her process of awakening has resulted in feelings of loneliness. I ask Peach how she deals with this tension in her friendships and she responds:

I don't know, I'm having trouble with it... And it's like... my love for this person and my hate for oppression... I don't know how really to reconcile those things... 
Well then [I think] I'm gonna cut this person out of my life because I don't feel safe around them, I feel like it's a toxic relationship. And there's times that I feel okay about doing that. And then there's these people where I'm like, I still have this love for you that isn't overcome by these other things that I'm having a hard time with and I haven't found a solution other than interrupting anyway... but I'm still struggling with it.

Peach considers just cutting individuals that cause dissonance out of her life, as Sparkles and Violet claim to have done, but this is not a solution for her in that she feels closeness with these individuals. The incongruence is so intense for Peach that she no longer feels safe in these relationships. Peach also claims that she has not been successful in her attempts to deal with the tension.

Elizabeth also describes the tensions in relationships that result from the interrupting. However, her way of coping is to avoid talking about certain topics. She describes this ongoing state of dissonance in her friendships:

Sometimes I would try to relay what we had experienced, like the revelations we had in class and I would try be really excited and the excitement level wasn't met... and I think that was the really frustrating part was there would be a lot of times when, 'oh my gosh, did you know this? Like, can you believe this?' I was shocked, and it would be like, oh, whatever... oh you don't have this passions, but I feel like everybody should, so which happens a lot, I'm sure with like new information for people that get so excited and want to share and people just don't function the same way but I don't know if it was maybe it does come differently from [the instructor] who has all the information, I mean just taking the class, I mean people don't see it as valid.

Elizabeth has a desire to share what she learned in Girl Power with her friends that have not taken the course but when she does this she becomes frustrated because she is excited and passionate about the material but those outside of the class do not meet these feelings. Elizabeth hypothesizes that her level of excitement and passion about the course may come from being in the class, particularly from the instructor. Here Elizabeth 
portrays the instructor as an expert whom has all the information and so when the information flows from her it is valid. In contrast, Elizabeth positions herself as less than an expert. As a result, Elizabeth concludes, people don't "see it as valid"

Participants seem to try to deal with tension in friendships by first trying to interrupt those friends. If that does not work, some resort to cutting people who do not respond out of their lives. Other participants deal with dissonance in relationships by avoiding areas of disagreement.

Dissonance with men. The participants experience dissonance with various men in their lives as well as with same-sex friends and family. These men range from husbands and boyfriends to their male friendships and men that the women were attracted to. Sparkles discusses her changing relationships with her boyfriend during and following Girl Power:

I was trying to develop this new lifestyle of interrupting oppression, not using oppressive words, and all of these things and when you're starting to do that and you use oppressive words you feel so bad about yourself, like 'oh my god I can't believe I just used this word' or if I would hear... my boyfriend says things like that and I would try to talk to them like hey, we're learning about this in class have you ever thought about this and put it in the words that [the instructor] would put it... [he would say] 'I'm just gonna talk about whatever I wanna talk about' [that] was hard.

Sparkles expresses frustration that her boyfriend does not respond positively to her attempts at interruption. She initially does not consider that it may be hard for her boyfriend to deal with what she is telling him. Sparkles then explain how she deals with this conflict:

Like if my boyfriend would use oppressive words I would just be 'okay, I don't like when you say things like that and this is why' and most the time he' $\mathrm{d}$ be, 'oh yeah, you're right sorry', or if he would just be like, 'oh god, again, just leave me 
alone, let me say whatever I want to say', I'd be like, 'okay, but you're better than this' or I would, at first just get angry, why aren't you listening to me? And then I just started thinking, okay it took me a while to internalize this material so [I'm] gonna keep feeding it and hopefully in a couple months it will become internalized and I feel it did and now it's way better and different.

When asked to reflect more on her response Sparkles does acknowledge that it took her a while to accept the material in Girl Power. She recognizes that her initial response of anger and frustration with her boyfriend may have not been helpful in moving him along, and attempts to adopt a more tolerant and patient position.

When I ask Violet if she experienced any conflict in her life during or after Girl Power she responded:

Yeah, I mean, my partner for one. I'm still just like drilling him about things, I'm sure he things I'm a shrew or a pit bull but there are things, the poor guy has to have me practice on him every little thing, he's sort of like, he was raised very differently from me so, I think there are things that he knows to be true in his heart of hearts but he hasn't worked on that whole conditioning, to say, so I'm on him like flies on crap ((laughs)).

Violet talks throughout the interview about her struggles with her partner. In this excerpt, she describes herself as policing and practicing on him. She does not elaborate on his reactions to her interventions, although she attempts to explain his behavior by referencing the home where he was raised. Violet does seem to have a sense of humor about the situation though, which maybe helps her deal with the dissonance and conflict involved in this relationship.

I asked Ruby if she experienced any conflict between what she was learning and doing in Girl Power and other aspects of her life and she responds:

Yeah with men, I mean ((sort of laughs)), cause after Girl Power and after other Women's Studies classes I became this little feminist ((laughs)) so all my guy friends, they like it but they don't like it, but I don't care its not about them its 
definitely about me... I don't give a damn that somebody doesn't like that, ((laughs)) I don't care if you feel like I'm trying to be a man, I'm not trying to be a man, whatever that means, so ((laughs)) stuff like that, guys are just like, 'you need to go back to being this sweet little woman' and I think its just they know that stuff gets on my nerves they'll say stuff like that to purposefully annoy me, so I just don't even entertain it, I'm just like whatever.

Ruby acknowledges that the classes she has taken over the past year have changed her into a "little feminist" and notes that her male friends have noticed but not responded well to this change. She laughs throughout this part of the interview, perhaps because laughing is a way of acknowledging her own discomfort. She also dismisses many of her male friends' reactions by suggesting they are not serious and instead just say things to irritate her.

Overall, the participants tend to deal with the dissonance they felt with male partners and male friends by trying to interrupt them. The responses to interruptions seemed to vary. Some men responded positively to being interrupted at times but responded defensively at other times. Violet and Sparkles seem to suggest that they were able to "bring along" their partners in their process of awakening, although this process was not necessarily a smooth one. As with other sources of dissonance and motivation for interruptions much of the tension seemed to allude to the men using oppressive or sexist language. However, it is unclear what language was being used and if other perceived transgressions were involved.

Dissonance with family. Participants did not often address family relationships. But when the topic of family did arise, it typically was identified as a site of conflict. For Elizabeth, the idea of talking to her family about what she learned in Girl Power is nonsensical she states, "I don't even really tell my mom that I take Women's Studies 
classes." At other points in the interview, Elizabeth talks about the fact that the culture she grew up in and the culture of Girl Power are very different. Just as she tries to avoid conflict with her friends by not discussing certain issues, Elizabeth avoids conflict with her family by not telling them what she does in school. Remi expresses a similar sentiment:

Talking to my parents sometime, they have no idea what really Women's Studies is, so my mom says weird stuff sometime to me ((laughs)). And then it's weird to be a person who I've immersed myself in this type of material and ways of thinking and theory and then real life isn't like this ((laughs a bit)), so I can try hard to like change it I guess, within my own bubble but its still hard ((laughs a bit))

Throughout the interview, Remi often uses the term "weird." She sometimes seems to use the term to describe people who are being oppressive or offensive, and other times as a reference to conflict or dissonance. In this part of the interview, she seems to use the term in both ways. First, she discusses not being able to connect with her parents around what she is learning in school and then continues by discussing the dissonance she feels by being involved in critical and feminist inquiry in school but knowing that these ways of thinking are less accepted in broader society. Remi and Elizabeth both choose to not share their changing view or understanding the world with their family. This decision could suggest that they have not fully integrated this new way of viewing the world into themselves. Alternatively, they may just wish to avoid conflict with their families. Whereas participants have the option of leaving friendship groups that perpetuate what they view as oppression, the option of leaving family may feel more difficult or traumatic.

Violet discusses how Girl Power made her family experience: 
... I started thinking about things that he [her father] would say to me when I was younger and it wouldn't be like, you are this way, it would be more like [all] women and it was sort of horrifying that somebody... that intelligent and worldly... would think that women were really not as worthy as men. So then I'm like oh great, how much of that did I soak up in childhood that I'm still harboring.

The course caused Violet to interpret her father's actions and words differently than she had previously. After the course, she feels she sees sexism but this contradicts her view of her father as "intelligent and worldly" and thus unlikely to express sexism. These revelations also cause Violet to be concerned about how his attitudes may have influenced her in ways that she has not understood or noticed before.

Overall, participants seem less likely to interrupt family members, as compared to friends and partners. The most favored way of dealing with dissonance within the family centered on making certain topics off limits for discussion, thus avoiding the areas of tension. Alternatively, participants dealt with dissonance in other areas using a wider variety of strategies, including avoiding relationships and situations viewed as oppressive. While participants' efforts to change some of these settings suggests engagement in critical action, a core component of critical consciousness, participants also have difficulty integrating "good" and "bad" aspects of people, and dealing with their own sources of ambivalence. I return to participants' struggle with these sources of tension in the next chapter. 


\section{Chapter Six: Discussion}

This dissertation began with background to my longstanding interest in critical consciousness. Long before entering graduate school, I have been interested in the question of how to go about promoting social change. As I pursued this question through my graduate studies, I became convinced that the social change methods utilized by many activists were inadequate in fully addressing the scope of emerging problems. Although these methods address the social and economic nature of oppression, they do little to address cultural and psychological effects. While it is important to attend to structural aspects of oppressive systems, it is also necessary to attend to the psychological effects of these conditions. Therefore, the question became one of how to go about promoting progressive social change at multiple levels. I began an ongoing investigation into theory and practice that attends to the psychological and cultural effects of oppression.

Although my interests have developed over the course of my graduate studies, I was committed to trying to promote and understand critical consciousness after my first reading of Paulo Freire's (2000) Pedagogy of the Oppressed as an undergraduate. According to Freire, dialogic and experiential education, also termed Critical Pedagogy, can be used to develop critical consciousness. The model laid out by Freire seemed to address the structural and psychological effects of systemic injustice on both the oppressed and oppressor. Freire not only addresses the problem but also proposes an eloquent solution. His method of transformation offers a powerful demonstration of how to create psychological and social change with oppressed groups while non-violently working with oppressors. 
My commitment deepened as an undergraduate when I spent the summer as a facilitator for an education program for "youth on the margins" that utilized a Freirean approach. Critical consciousness and Critical Pedagogy became real as I witnessed their transformational power for the youth, myself, and the other facilitators. During this same period, I spent time with my university's service-learning coordinator and I began to theorize that an ideal path to critical consciousness involved a combination of Critical Pedagogy and community engaged learning. I also stumbled upon the field of Community Psychology as I searched for a potential academic home. The field seemed liked the perfect place to pursue socially relevant research that worked with oppressed groups. Additionally, Community Psychology was an ideal avenue in which to pursue research related to critical consciousness, as detailed in the introduction chapter.

One of the reasons I chose to study at Portland State University was its unique and innovative commitment to community engaged research and university-community partnerships as exemplified by the University Studies Senior Capstones. These capstones require all students to take a course that utilizes community service-learning. While multiple outside sources directed me to the Girl Power capstone as an ideal environment to conduct my study, this setting also fit with my experiential knowledge regarding the development of critical consciousness. Together my personal activist experiences and disciplinary training in Community Psychology led me to study critical consciousness as a primary phenomenon of interest. Additionally, throughout my graduate education I have been exposed to feminist and psychoanalytic theory through my advisor and my minor studies in Sociology. I have integrated theoretical insights from these theories into 
my psychological study of critical consciousness in order to analyze the understudied but essential components of emotions and relationality. The intersection of these experiences and bases of knowledge (i.e., praxis) have played a role in all stages of the present study.

\section{Reflexivity and Countertransference in the Research Process}

The methods of participant observation, interviewing, and qualitative data analysis involve interpreting findings through multiple lenses. Self-reflexivity can add to the trustworthiness of findings, which is one of the primary markers of good qualitative research (Stiles, 1993). I strove to be continually aware of how my life experiences, gender, sexual orientation, class, ability, and race affect how I interacted with my phenomenon of interest and research participants. By using action and participatory research strategies, Critical Pedagogy, and qualitative methodologies, I hoped to avoid oppressive research tendencies while remaining cognizant of power dynamics attendant to any program of inquiry. To obtain trustworthiness I engaged in a number of suggested measures, including self-reflexivity, openness regarding possible personal biases, prolonged engagement with the material during and after data collection, and a process of peer debriefing. Additionally, I meet with my dissertation chair throughout the process to discuss my developing understanding of the data. Snow, Grady, and Goyette-Ewing (2002) also suggest that it is vital for community psychologists to have a strong awareness of personal values and how these influence their work in the field. I filtered every act I observed through my own experiences and biases. Even when striving for objectivity, researchers inevitably bring subjectivity into their programs of inquiry (Fine 
\& Vanderslice, 1992). It is important, therefore, to acknowledge and confront the experiences and biases that may affect my study (Tolman \& Brydon-Miller, 2001).

Reflection is a key aspect of Freire's theory and served as a means of data collection. Following each session of participant observation, interviewing, and data analysis, I kept a research journal that chronicled my reactions to the material and the overall experience. Further, this notebook served as a place for me to record my developing understanding of the data. I reviewed field notes, journals, and transcripts multiple times during data collection and analysis in addition to meeting and debriefing with graduate student peers and my research advisor to discuss my developing interpretations of the data.

This section takes up my subjective experience in carrying out the research, specifically through the psychoanalytic lens of countertransference. The concepts of transference and countertransference allow for the researcher to "weather the inevitable tensions and volatile emotions that arise: sadness, fear, hostility, hatred, tenderness, desire" (Haaken, 2008, p. 205). Transference and countertransference describe the way in which both the researcher and the participant carry a history of experiences that play out during their interactions and can affect the research process. Attending to those dynamics allowed room for understanding anxieties and defenses that emerged in the research process and provided a tool to avoid defensive distancing (Haaken et al., 2011).

When applied to the research process, the concept of countertransference can help in recognizing the latent or subtextual meanings of the data. Countertransference involves not only understanding how past life experiences and identities affect the 
research process but also how the theories used impact this process (Wachtel, 2011). Wachtel (2011) also advises that countertransference often occurs when ambiguity is highest and can be tracked by attending to the words one chooses to express themselves and communicate to therapeutic clients or research participants. Attending to my actions and words as I conducted this project allowed me to understand when and how countertransference played a role in the study development, data collection, and data analysis.

As a participant observer in the Girl Power course, several activities stirred strong emotional reactions in me during and after the class. I experienced a particularly strong physical and emotional response to the identity circle activity. During the activity, I felt shaky and my hands were sweaty. Following the activity I started crying over a simple frustration. I think the basis of this reaction was twofold. First, it was a response to hearing stories that are hard to hear. Even though I am aware of the statistics around sexual assault, eating disorders, and suicide attempts (for example), it is hard to learn that those around you have struggled with or experienced these issues. Second, I think that I felt some uneasiness and guilt in my role as researcher during this activity. Although I fully participated and did not take notes during the activity, I experienced myself as a voyeuristic presence during this aspect of the course. This struggle between being a participant and researcher in the course continued as I analyzed my data and struggled in the beginning in drawing out the potentially regressive aspects of the participants, many of whom I had grown fond. 
The course stresses the importance of rap sessions and suggests that they are the most transformational aspect of the course. I felt that I had failed in a sense because this was not the case for me. There was a large age and experience gap between the rap session participants and myself. One barrier for me, as a white woman, centered on how to talk about race and oppression with a group of young Latina women. While I was able to speak broadly about race and ethnicity in an academic sense, I was not able to engage participants on a personal level regarding the specific struggles they faced as Latina youth. Lack of identification and connection with rap session participants may be a barrier to having an immediately transformational experience. Further, my guilt for not having a powerful experience in the rap session suggests a demand characteristic of the course, something I saw reflected in some of the participants.

I developed positive affiliations with some participants and more negative associations with other class members. These relational valences influenced my analysis of the data. I often struggled to find "useful data" in interviews of participants with which I had a more negative response. This was particularly brought to my attention when I went over an interview of negative association with my dissertation chair, because I did not see the interview as very useful. However, my chair was able to guide me to find complex narrative threads in the data that I had overlooked. As a group, however, I felt a strong responsibility to the participants, even those with whom I had developed a more negative reaction.

Throughout the research process, I struggled with awareness of being critical at times of the responses of my participants. It took conferring with and support from 
graduate student peers and my dissertation chair to analyze the areas of the data that could be viewed as critical of the participants, as well as findings that implied criticism of the Girl Power course. The experience of attending the course led me to develop deeper investments than if I had just interviewed the participants. Further, I experienced Girl Power as a pleasurable course that aided my continued SPC consciousness development, potentially hindering my ability to see problematic aspects of the course. While I believe my involvement in the Girl Power course aided in rapport with participants and the quality of the data I collected, this same close involvement did, at times, inhibit me at the level of interpretation of my findings. Further, I was over committed to the outcome of SPC consciousness development and at times saw support for this outcome when maybe another interpretation of the data was more appropriate. However, through reflection, debriefing, and continued engagement with the data I worked to understand how these investments impacted me and thus expanded my capacity to analyze multiple interpretations of the data, some that supported progressive SPC consciousness development and others that supported more regressive capacities.

Additionally, I struggled with how to share results with research participants. I have immense respect for the participants and wanted to portray them in a positive light. However, I came to realize that over-simplifying the complex feelings and thoughts of the participants would be an injustice to them in its own right. Further, by not drawing out the problematic aspects of the Girl Power course, I lost a potential opportunity for my research to be valuable as it could lead to ways of improving the course and other efforts aimed at enhancing critical consciousness. Later drafts of the findings and discussion 
sections integrated more critical interpretations of the data. In working toward this aim, I have tried to attend to what Stein \& Mankowski (2004) describe as the need for researchers to consider the effect of their research findings on the participants at the time of the research project and into the future. As I analyzed and wrote up my findings I tried to maintain consideration for the impact this dissertation could have on the participants and on the course instructor. Within these words and their interpretation are the lives of real people; I strove to balance honoring their stories with drawing out areas for expansion and analysis. I hope that the areas of critique of the course I have drawn out can be used to improve the courses, and other courses like it, in the future.

I carried certain expectations to this project based on my fieldwork, prior experiences, and activist commitments. I choose the Girl Power course because it was ideally designed to facilitate critical consciousness, thus I expected to see some change in participants' level of SPC consciousness. While there is evidence that participants did undergo a process of development, which mirrored what was expected based on my theory of SPC consciousness development, at times I may have been overcommitted to this outcome and had to step back and debrief with peers and my research advisor to check my interpretations of findings. Often these conversations led me to interpret the data in multiple ways and to attend more carefully to the potentially problematic nature of the participants' comments and to the structure of the course.

My personal history of activism and critical consciousness development also influenced my developing understanding of the data. While such "insider status" can provide useful insight into data interpretation, it can also become problematic. My 
personal history with critical action had centered more on large-scale protests and boycotts as opposed to the practice of interpersonal interruptions discussed in the course. This led to one area where I was potentially overly critical of participants - the fact that their critical action did not seem to extend beyond interruptions. However, as I was discussing my interpretation with a graduate student peer, it was brought to my attention that a range of barriers contributed to engaging in critical action. These barriers are discussed more in later sections.

Writing the findings and discussion sections has led me to ask myself the questions I asked of my participants. I realized that despite my critique that interruptions are limiting, I found myself interrupting oppressive language more often after my Girl Power experience. I have been working to interrupt myself and those around me from using words like "bitch" and "crazy." Additionally, I have found that my focus on emotions and relationships in this study has changed my teaching strategies. This study has sensitized me to the role of emotions and relationships in my teaching practice, leading me to integrate and attend to these dynamics more effectively into classroom discussions.

Throughout this research process, I have tracked my subjective responses to the data. At times this has been challenging given that reactions are sometimes undesirable and at other times less consciously articulated. Combined with peer and mentor debriefing, this process has brought greater clarity and focus to my interpretations and helped me to recognize blind spots in my interpretations as well. The theoretical interpretations of the findings that follow result from not only the application of my 
theoretical framework but also my process of reflexivity outlined previously. Further, understanding the process of countertransference in the research process not only allowed me to account for some of the ways that my personal biases, experiences, and unconscious desires inhibited the analysis of my data but also enhanced my ability to theoretically engage with the data through my own understanding of the processes involved.

\section{Theoretical Interpretations}

The dissertation pursed two primary research questions:

1) How are emotionally and relationally significant Girl Power experiences related to Social-Political-Cultural (SPC) consciousness development?

2) What tensions arise between the dominant culture and/or significant others' values and the values of the Girl Power capstone and how do these tensions move individuals toward or away from critical consciousness?

These questions directed the data collected, as well as the analysis and interpretation of that data. The findings were grouped under two themes. First, the processes of awakening theme addressed participants changing conceptions of themselves, others, and the world around them and the role of Girl Power in these processes. This theme addressed the first research question. Second, the sources of dissonance theme addressed the internal and external tensions that participants faced during and following Girl Power. This theme helped address the second research question.

My model of critical consciousness development is based on three levels of SPC consciousness. Individuals may move forward or backward on this continuum based on 
internal and external experiences that enhance (i.e., movement towards critical consciousness) or hinder (i.e., movement towards pre-consciousness) capacities for critical reflection, critical efficacy, and critical action. The middle level of SPC consciousness, transitional consciousness, is a time of marked dissonance and strong emotions as participants began to transform and integrate old ways of being and acting on the world into critical consciousness. Additionally, each of the three levels of SPC consciousness is associated with cognitive, emotional, and behavioral characteristics. As discussed in chapter three, the theoretical drivers of SPC consciousness development include social support, role models, dialogue, exposure to diversity, managing deviant and limiting emotions, enhancing emotional energy, labile and collective nature of emotions, and dissonance.

Progressive drivers of development facilitate movement towards critical consciousness. Progressive capacities include the ability to tolerate ambivalence and conflict as well as the development of "psychic capacities to integrate infantile relational ties and egoistic strivings into a larger sphere of attachments and ethical commitments" (Haaken et al., 2002, p. 66). Regressive tendencies, on the other hand, involve "psychological merger with a group ideal and diminished capacity for critical distance or reflection" (Haaken et al., 2002, p. 66). Through the discussion, I attempt to draw out how Girl Power and the participants express both regressive and progressive developmental capacities by highlighting some of the key areas of tension participants experienced during and following the completion of the course and the role these tensions played in participants' SPC consciousness development. 
Magical thinking. Both in-class activities and rap sessions influenced participants' critical reflection, critical efficacy, and critical action components of critical consciousness. Further, the course's focus on building relationships contributed to participants' movement towards critical consciousness through building community and identity. These findings held for students that came into the course with relatively low and high levels of SPC consciousness ${ }^{2}$, although the processes and focuses of these two groups differed. The transformational experience in the class was problematic, however, in key ways. Participants often talk about their experiences in Girl Power as similar to a religious conversion. Freire (1993) argues that when exposed to Critical Pedagogy individuals will ideally move from magical consciousness, where a person accepts the phenomenal and natural inevitability of the social world, to naïve consciousness, which involves a greater but still limited capacity to interpret social, political, cultural and historical determinants. Ideally, through dialogue and critical education, naïve consciousness is transformed into a more complex critical consciousness. However, at the stage of naïve consciousness there is also the possibility that individuals may regress into fanaticized consciousness, characterized by irrationality, disengagement, and fear of freedom (Freire, 1993). In this state thinking becomes heavily one sided and dialogue across differences becomes difficult. These reasoning characteristics mirror the religious fanaticism common after some conversion or "born again" experiences. These experiences of conversion are often accompanied by decreased ability to deal with

\footnotetext{
${ }^{2}$ Participants' level of SPC consciousness before, during, and after the course was based on their self-reports in this area. Also, characteristics of the levels of SPC consciousness as identified in the theoretical framework were noted.
} 
ambivalence, increased fear of conflict, and a blind acceptance (or faith) in authority figures perceived as "good" or representatives of a higher power.

Further, in a fundamentalist conversion, the excitement generated by the new way of seeing and understanding the world may lead to seeking out only those that agree with the new worldview. Old ways of thinking are split off from consciousness and regarded as bad rather than being integrated into the new way of viewing the world. Dialogue with those with alternative points of view is diminished, as is the ability to critically think about different points of view. In short, those in this state of fanaticized consciousness, whether based on religion or politics, may become reactionary and lose the ability to think dialectically. This reduced capacity for cognitive and moral complexity is the opposite of what is expected when critical consciousness is enhanced (Mustakova-Possardt, 1998).

The awakening metaphor enlisted by participants and taken up in this dissertation suggests some dramatic change or transformational movement. However, the nature of this movement is not always clear. It may suggest movement toward critical consciousness or it may suggest a quasi-religious conversion experience. At times participants take on the language of fundamentalist religious groups. This language does not suggest dialogic thinking and movement towards critical consciousness but rather blind acceptance of a newly understood truth. Students also project what could be interpreted as a fanatical consciousness when they talk about only wanting to surround themselves with those who support their new ideas and language. While it is important to 
have community that supports growing levels of critical reflection and offers opportunity for critical action, limiting oneself to fellow believers may produce insularity.

Further, participants' process of awakening is also problematic in that it does not seem to integrate past experience and thoughts into new understandings of the world. A more developmentally advanced form of transformation would more fully incorporate past experiences and past selves with the newly developed views and self-representations. Totally eschewing all old ways of knowing and acting sets students up for difficulties. Isolation may result, as participants are no longer able to receive social support from family and friendship groups. Difficulties may arise when students see people they care about (or themselves) engaging in behaviors they view as oppressive. It is hard for participants to reconcile fighting oppression and loving those who continue to engage in oppression.

Yet, the overall content of responses does not suggest exclusive development of regressive tendencies. Some participants discuss integrating old and new ways of understanding the world. Participants also talk about being in dialogue with those outside of Girl Power and with those engaged in other areas of social justice work. Finally, participants demonstrate movement towards critical consciousness in their widening frameworks for thinking about injustice and engagement in critical action.

The theoretical framework for this dissertation highlights that SPC consciousness development is a complex process. The findings from this dissertation suggest that this process of development may serve to contain dissonance and anxiety. Through stressing critical consciousness development as an ongoing process, the Girl Power course offers 
potential protection against fanatic consciousness. When students are guided though development as a process, they can deal with the anxiety of falling short of their expectations and may be less likely to engage in psychological splitting that can lead towards magical thinking and fanatic consciousness.

Experiential and academic content of Girl Power. Previous investigation of critical consciousness has tended to avoid relational and emotional dynamics in focusing singularly on cognitive factors. Emotions are often viewed as hindering the process of critical consciousness development by prohibiting growth of critical reflective capacities (Carlson, Engebretson, \& Chamberlain, 2006; Freire, 1993). However, a more complex view of emotions proceeds from the premise that they can be central to critical consciousness development as well as limiting critical consciousness development. One of the aspects of the Girl Power course that led me to select it as a site of my dissertation was its focus on emotions and relationships. Emotions and relationships are often omitted from research on pedagogy, even in courses devoted to developing critical thinking. Many teachers are taught to fear emotions in the classroom as antithetical to process of rational inquiry. Emotions are also feared because they represent a seemingly unmanageable energy that may threaten traditional classroom dynamics of order and logic. Finally, some instructors feel they are not trained to handle the emotions that may results from some classroom experiences. However, if emotions and relationships are a key aspect of critical consciousness development, these aspects of mental life must be incorporated into our pedagogical models. Further, emotions and relationships are 
present in any context, including the classroom, whether instructors choose to acknowledge their presence or not.

Participants in the study noticed and appreciated the attention the Girl Power instructor gave to developing relationships and to working with emotions. Participants noted that the ability to transfer the information from the purely cognitive and academic to the emotional and personally meaningful seemed to enhance their ability to engage in critical reflection. Further, the experiential knowledge gained through the servicelearning component (i.e., rap sessions) was central to how many participants described SPC consciousness development.

The aspects of the Girl Power experience that were most meaningful for participants varied, depending on previous life experiences and their prior level of SPC consciousness. For some participants the emotional and relational aspects of the course were most important to their continuing SPC consciousness development. These aspects of the course were important for students who had previously taken Women's Studies courses that lacked an emotional or relational pedagogical focus. Other participants found the academic content of the course (e.g., readings and discussion of theories of oppression) particularly helpful in enhancing their SPC consciousness. These students often had previous personal experiences with oppression or exposure to marginalized groups but did not have the vocabulary or theoretical understanding to fully integrate or process these experiences. Other participants found the community engagement component particularly helpful and found the academic content "came alive" when they applied the knowledge they learned in the classroom to the rap sessions. Participants 
with this experience often found themselves connecting with and/or identifying with the girls in the rap session.

While Girl Power was not the primary impetus for SPC consciousness development for some participants, it played an important role in continuing to move them along the SPC consciousness continuum. The course worked through a variety of mechanisms in allowing participants at different starting places along the continuum to gain something productive from the course. These mechanisms included normalization and emotional energy.

The process of normalization of experiences (Summers-Effler, 2002) is an important aspect of SPC consciousness development as it enables individuals to understand that their personal experiences hold some validity in the development of critiques of society. This process of making the personal political is an important component of the critical reflection element of SPC consciousness. Normalization also validates emotions by making emotions previously viewed as personal and/or deviant part of a broader political structure. The communal and non-judgmental space built into the Girl Power course allowed some participants to express emotions that the dominant society might deem deviant, anger for example. Not only are "deviant" emotions allowed to be expressed but they also become acceptable, this process frees up cognitive processes that were previously dedicated to managing emotions and can now be used to develop critically reflective cognitive capacities (Summers-Effler, 2002).

The emotional work of the course occasionally took on a transformational tone. Through the activities and relationships built into the course, emotions that limit SPC 
consciousness development; for example, fear, guilt, and shame, could be transformed into emotions that enhance development such as, hope, love, and anger. The course seemed to help students overcome fear and to learn a concrete and applicable way of dealing with oppression. These emerged as major factors contributing to students' ability to apply their process of awakening.

Emotional experiences tied to the course were not static. Many students found that the course was both exciting and draining, a vacillation of emotions that fits with Hoggett's (2009) focus on the labile nature of emotion, which he theorizes as central to the empowering capacity of emotions. Hoggett argues that the dynamic coupling of opposite emotions (e.g., love/hate, despair/hope) allow for emotions to become a driving force for efficacy. Thus, the course not only normalized emotions but also worked to transform them.

Emotional energy was another important component of the course that contributed to the process of awakening. Emotional energy is:

.... a feeling of confidence, courage to take action, boldness in taking initiative. It is a morally suffused energy; it makes the individual feel not only good, but exalted, with the sense of doing what is most important and most valuable... feeling of emotional energy has a powerful motivating effect upon the individual; whoever has experienced this kind of moment wants to repeat it. (Collins, 2004, p. 39).

The concept of emotional energy helps understand dynamic connections between feelings, efficacy, and motivation (Summers-Effler, 2002). The Girl Power course is designed to enhance emotional energy through a number of practices. The high levels of emotional energy experienced during the course and during reflection back on the course appear to enhance participants' movement along the SPC consciousness continuum. 
However, emotional energy is not in and of itself a progressive force. Emotional energy has the capacity to drive individuals to less advanced moral and thinking tendencies. Emotional energy is very high in many cultish experiences; the individuals involved in these experiences often exhibit movement towards regressive thinking tendencies such as magical thinking, blind acceptance of authority, binary splitting, and fanaticism.

Another important component of emotional energy is its role in strong relational ties. Emotional energy is enhanced through interpersonal connections and collective identity formation (Summers-Effler, 2002). The role of emotions in relational development is also emphasized in another theoretical touchstone for this dissertationthe work of Carol Gilligan. Gilligan (1982) theorizes that the ethic of care is central to female development and to building and maintaining relationships as a key component of moral development. Based on my data, this seems to hold true for critical consciousness development. The relationships built in the course-with the instructor, rap session participants, and with other class members-are a key driving force for movement on the SPC consciousness continuum. Students with stronger relationships, particularly in the rap sessions and with other course members, appeared to have experiences that are more meaningful in the class. Further, the emotions and relational aspects of the course seem dynamically linked. The parts of the course that tended to elicit strong relational bonds also tended to stir emotions. These emotions in turn often enhanced relational ties.

A number of participants stated that they preferred to avoid conflict whenever possible. Additionally, dialogue in the class itself was often viewed as rife with tension. Participants preferred that everyone "get along" and agree. This tendency does not leave 
room for critical dialogue, a primary means to, and result of, critical consciousness development. This fear of dissonance, conflict, and aggression seems to mirror Gilligan's model of female moral development in its focus on an ethic of care as opposed to an ethic of justice.

Gilligan (1982) presents a feminist critique of the work of Lawrence Kohlberg, which exclusively focuses on morality as centered on an ethic of justice. By focusing only on justice and independence as the basis of advanced moral reasoning, Kohlberg overlooks the equally important capacity for care and interdependence. The ethic of care stresses relatedness and the value of everyone having a voice. The participants' aversion to conflict represents a problematic tendency in Gilligan's moral framework. An ethic of care alone does not account for the full range of moral reasoning that is needed to address oppression. While the stress on community is important to building solidarity and critical consciousness, an ethic of care alone does not necessarily motivate individuals to work toward greater equality. An ethic of care integrated into an ethic of justice offers the strongest moral ground for confronting relations of domination in all aspects of life. Many of the participants in this study seem to have developed a strong ethic of care but lack an ethic of justice, limiting their ability to engage in diverse critical actions.

Polletta (2002) situates the inability to tolerate conflict not within the individual but within the social setting and process of participatory decision-making. She notes a similar inability to deal with conflict in many of the social movement organizations she studied. When discussing this weakness of the participatory form Polletta (2002) states:

When a groups has common interests, consensus-based, face-to-face, and egalitarian deliberation can deliver good decisions... When member's interests 
diverge, however, practices that were intended to express and strengthen the group's shared purpose must protect individual interests against those of the majority or the powerful, and they cannot do this. If the groups... do not bring in some features of conventional adversary forms, they end up simply pressuring people into line... The participatory democratic dilemma is how to choose a 'group' interest from among competing individual ones. (p. 15)

In other words, the participatory form does not have the capacity to fully address the interpersonal conflicts that invariably arise. The situation becomes even more complicated when relationships among the group are taken into account. The inability to resolve this conflict was a source of frustration for participants in the course and represents an important dimensions for groups that utilize this style of deliberation to consider and deal with in the future, including Girl Power. However, it is important to note that despite these limitations this style of decision-making does allow more room for expressive and relational dimensions than more hierarchical and less participatory approaches.

Girl Power as therapy and consciousness raising group. Enlisting experiential knowledge in the classroom can become problematic. Emotionally evocative learning experiences can illicit overzealousness or fanatical consciousness-a form of thinking that precludes the development of dialectic reasoning required in critical consciousness. Participants sometimes express the grandiose belief that interruptions and altering language use alone can change the world — a belief rooted in what Freire (2001) calls magical thinking, a mode of thinking that is counter to critical consciousness.

The focus on emotions and relationships in the course can also lead to students emotionally investing in the experience in ways that require additional outside resources. Many participants described Girl Power as draining and two participants stated that they 
needed to seek outside therapeutic help in dealing with the challenging nature of the course. These participants had the resources and connections to gain these outside resources in that they were already seeing therapist. However, other students may have not had access to therapy when the course became overwhelming. Students that had this taxing experience in Girl Power but did not have the resources to cope may have been less likely to respond to my request to talk about the course, making this experience potentially underrepresented in my data.

Emotionally and cognitively withdrawing or distancing from a setting is one way in which individuals may express resistance to some aspect of that environment (WallinRuschman, 2011). This is particularly true in a setting, such as an academic course, that has punitive repercussions for physically or totally leaving the environment (e.g., receiving a failing grade, losing money or financial aid, delayed graduation). Given the negative consequences that may limit students' ability to withdrawal completely from the situation, it is important to consider the ethical implications of creating an emotionally intense and demanding environment in a classroom setting. While Girl Power does try to provide some resources to students in helping them cope with the evocative experience, for example, time for debriefing, relationship building, access to mentors and the instructor, these resource may not be enough for some students.

Some participants did talk about the Girl Power course as a therapeutic experience. Participants often described the course as emotionally reparative and placed the instructor in the role of therapist. This is problematic for an academic university course, not only because instructors are not trained counselors but also that the role of 
grades in the course precludes a therapeutic environment. Future iterations of Girl Power could integrate resources to help students deal with the emotionally taxing aspects of the course. Further, it is important for instructors of course of this nature to work on clarifying the boundaries between the classroom and therapy session. These settings offer distinct contexts, roles, and goals that require their separation in order for each to be maximally effective.

While Girl Power utilizes many of the strategies developed in activists and other consciousness raising groups, it is important to note that the classroom has important limitations that are not present within these community groups. In addition to the financial commitment, the university classroom also maintains a level of authority for the teacher. Although instructors committed to Critical Pedagogy often attempt to diminish the distance between teacher and student, the teacher still maintains the most control over the classroom because of the assignment of grades, development of the syllabus, and control of classroom time. Many consciousness-raising groups strive for a complete lack of hierarchy that is just not possible in the classroom setting.

The two areas of relationships most dominant in the Girl Power course, that among the students and that between the student and instructor, also represent limitations of the extent to which class can be an inclusive and participatory space. Polletta (2002), in her work on social movements, suggest that many attempts at participatory democracy eventually fail because they over rely on models of interaction common in society, including: friendship and the relationship between teacher and student. Each of these relationships inhibits the fullest practice of participatory democracy. Polletta's 
suggestion is that we must find new models of interacting in society to overcome the limitations of these types of relationships. Some of the disadvantages of friendship as a basis of social change are noted in the Girl Power capstone. For example, some participants do not feel included or integrated into the capstone community, such as Violet as demonstrated when she recollects her story of not being supported in the identity circle.

Idealization of the instructor. Participants placed many desires, fantasies, and needs onto the instructor in the course. Often participants struggled to label the role of the instructor because she occupies a different position than do many other university teachers, and was seen as fulfilling many roles simultaneously (e.g., friend, facilitator, teacher, therapist, leader, coach, role model). Some of these roles conflicted. For example, a therapist that is also viewed as a friend can become problematic. Often these roles are projected — as a form of transference-onto the instructor by the students. As the embodiment of a worldview, the instructor readily served as a site of idealization. Idealization is a central and vital capacity for development. It drives development from a very young age and is often what brings people into social activism (Brenner \& Haaken, 2000). Idealization can become limiting, however, when it is "split off or repressed and thus emerge(s) in a primitive, unintegrated way" (Breener \& Haaken, 2000, p. 334). This process of idealization may result in seeing the idealized group or leader as all "good" and outside groups as all "bad", a blunting form of binary splitting. The focus on idealization can take the tone of a religious conversion experience, as Brenner and Haaken (2000) suggest, "Utopian ideals can be destructive in that they often do mobilize 
longings for surrender, and create hypnotic group ties based on suspension of critical thought" (p. 334). The idealization that occurs in Girl Power seemed to generate both progressive and regressive tendencies.

When analyzing the interviews I listened for the basis of idealization. Participants stated that their admiration of the instructor was important as she was able to bridge worlds (the Girl Power community and the dominant society) in a way that facilitated learning and expansion. However, participants' fears of conflict suggested that the idealization might have disguised domination (Brenner \& Haaken, 2000). The participants discussed the instructor in a way that suggested she possessed a sort of omnipotent power. They drew on this power when they engaged in interruptions outside of the classroom. Students may have difficulties developing capacities that do not depend on the energy and passion of the instructor, thus impeding further development of SPC consciousness after the conclusion of the course.

Summers-Effler (2002) argues that a charismatic leader can often be an important source of emotional energy that can drive development:

A charismatic leader emerges as a symbol of a high solidarity ritual. In becoming a symbol of the experience, they are repositories of high emotional energy, which enables them to bring emotional energy to situations that have little... Through emotional energy incentives, those who have already developed a raised consciousness can spread the experience of consciousness raising. They can bring it to those who have not developed a critical consciousness by instrumentally creating intense rituals to legitimize deviant emotions and build collective identity. (p. 58).

While a charismatic leader can bring emotional energy to settings that lack this integral ingredient of developing resistance and critical consciousness, this leader does not create emotional energy but rather transfers it from one setting to another through teaching the 
process of building solidarity and the rewards of investing emotional energy. However, it is possible in situations of high emotional energy for some individuals to benefit more than others. Often the charismatic leader is positioned to benefit from the investment of emotional energy from others; indeed this is one of the things that positions them to transfer energy from setting to setting. Not everyone may benefit equally from this investment. Additionally, reliance on a charismatic leader is dangerous in that the leader may abuse their power or the individuals in the group may over rely on the leader so that when the leader is gone they are not able to continue their engagement in critical reflection and critical action

One of the primary roles of the charismatic leader is in developing rituals that normalize emotions previously seen as deviant and to enhance collective identity. In Girl Power, the instructor seems to fill the role of charismatic leader. She facilitates emotional energy through the use of check-ins, mentorship groups, and dialogue. This suggests that these aspects of the course are essential to one of the driving forces of SPC consciousness development. The instructor also serves as a role model for how to transfer critical reflection into critical action. She teaches one area of critical action specifically, interruptions.

Interruptions as a form of critical action. During their process of awakening, students seek tools to put their altered view of the world into practice. The process of "interruptions" is something that students identify as particularly meaningful. As taught in the course, interruptions are primarily conducted in regards to language viewed as oppressive. Participants talk about engaging in interruptions in a variety of settings. 
These interruptions are seen as a small step in creating a better world. Further, interruptions are seen as preferable to other methods of direct political action because interruptions are viewed as less confrontational.

Many participants noted that they have continued to engage in interruptions on a regular basis following the course, suggesting that the process had become an integrated aspect of their identity. Participants mentioned that learning about interruptions gave them a sense of efficacy - a feeling that they could do something to intervene in injustice. Many participants viewed this practice as a powerful force for personal and political change. A sense of efficacy is identified by Watts and colleagues (2011) as one of the core components of critical consciousness development. Individuals are unlikely to engage in critical action if they do not believe that their action can produce some sort of effect. Polletta (2002) suggest that efficacy can be developed through learning the skills of dialogue necessary for participatory decision-making, such as that used in Girl Power. While participants sometimes found this process frustrating, Polletta (2002) argues it can teach important skills, including "[training] people to present arguments and weigh the cost and benefits of different options" (p. 10).

Individuals may begin to develop critical reflective capacity without a sense of efficacy. However, when the knowledge of how to intervene in the oppressive structures is missing (i.e., critical action) development of SPC consciousness may become stalled at the cognitive level. As people begin to see more injustice in the world, it can become harder to sustain feelings of efficacy. Without tools to intervene through critical action, individuals may eventually deal with their dissonance by avoiding exposure to instances 
of injustice. Participants expressed dissonance by wishing they could "take back" their new lens of the world. Yet if given the tools to engage in critical action students can continue in their process of development through the praxis cycle.

The Girl Power course succeeds where other critical consciousness interventions sometimes fail insofar as it provided participants with a site for enacting a process of awakening through action. The practice of interruptions are relatively easy to learn and can be practiced individually. In addition to interruptions, many participants developed a critique of popular culture and subsequently altered their engagement in cultural and related recreational activities to reflect this new level of awareness. However, interruptions may be limiting in that they only address oppression at one level. If participants had developed an enhanced capacity for critical reflection coupled with critical efficacy, I expected that they would move on to engage in other forms of critical action (e.g., direct action, advocacy).

However, scholars have noted the difficulty in engaging in structural change efforts. Watts and colleagues (2011) suggest that opportunity is needed for individuals to engage in collective action. In other words, individuals are not likely to engage in a protest, for example, unless the opportunity to do so arises. Not only does the person need to be made aware of opportunities to engage in direct action, they also need to feel welcomed and valued by others involved in these change efforts. Lack of access to communities of social action becomes a limiting factor to engaging in systematic change efforts. 
Critical action, in general, requires higher levels of emotional energy than does critical reflection or critical efficacy (Summers-Effler, 2002). Engaging in critical action can create dissonance with friends and family, and requires considerable emotional investments to manage or override these states of dissonance. Critical action is also visible to those with more power, therefore increasing the likelihood of negative repercussions. While developing critical reflection and critical efficacy are internal processes, engaging in critical action is a behavior that can be observed (e.g., engaging in a protest, interrupting oppressive language). By engaging in this visible form of resistance to the status quo, and the structures of authority that maintain these systems of domination, an individual opens themselves up for negative repercussions to this resistance (e.g., arrest, losing a job, losing a relationship).

Given this, it is less surprising then that interruptions were the only form of critical action made available in the course. The course focuses on language, relationships, and emotions as well as emphasizing the interpersonal and cultural aspects of oppression. Yet, this focus on the interpersonal is also a limiting aspect of the course. Future Girl Power courses would strengthen their capacity for building critical consciousness if they addressed critical action strategies beyond interruptions.

"You can't take it back." One recurring theme centered on the "burden of consciousness," the sense that once the participants had undergone a process of awakening, and the dissonance associated with this, they could not go back to their previous way of being. In other words, they felt they were stuck, for better or worse, with an altered view of the world. This is an under-discussed issue in critical 
consciousness development. Learning to see oppression, everywhere (e.g., in relationships, media, social structures, language) takes a toll on most individuals. It also creates dissonance in multiple areas of one's life. While dissonance can facilitate SPC consciousness development, it can also create anxiety, strain, and other potentially negative consequences. The strain is greatest when individuals do not have the knowledge and/or access to participate in change efforts within a supportive community.

Fine and colleagues (2003) discuss this problematic aspect of critical consciousness development in their work with women prisoners. They describe how helping to increase the critical reflective capacities of incarcerated women put these women in the difficult position of more clearly seeing the injustice of their situation. However, the women were severely limited in their ability to fight this injustice, possibly leading to feelings of hopelessness and impotence. While my participants are not as limited in their actions as incarcerated women, they have their own set of restraints in engaging in critical action. Students must continue to earn money in jobs that they may begin to view as problematic. They must also continue to deal with family members who espouse views they now see as oppressive. When the Girl Power course ends, students are left with few resources to deal with these new areas of dissonance in their lives. They are "stuck" with this altered view of reality where everything seems problematic but hard to alter.

On the other hand, participants' view that they "can't take it back" suggests that they cannot reverse gains in critical reflection. This counters the theoretical framework of this dissertation, which highlights that individuals can move forward or backward on 
the continuum of SPC consciousness as they move through life. It cannot be concluded based on the data available in this dissertation if participants or the theoretical assertions are correct.

Community post Girl Power. Participants struggled to deal with the dissonance that developed in their relationships with friends, family members, and partners. This was a strong source of tension in their lives. Participants dealt with this tension in different ways. Some stopped "hanging out" with people they viewed as oppressive. Another strategy was to avoid particular topics of conversation in order to keep the peace and avoid conflict. A further strategy was to try to change people in their lives through interrupting oppressive behaviors. This strategy worked for some participants over time, even as it generated tension initially in the relationship. Participants that were not able to resolve tension through one of these strategies expressed distress in feeling that they were not able to leave these relationships.

Dissonance in relationships can become problematic for SPC consciousness development over time. When students leave college, many no longer have access to the kind of open learning space and support that Girl Power provided. Many participants professed to want to continue to grow in their ability to recognize oppression and to fight injustice, but most participants did not directly express a worry about losing the support of the Girl Power course. Rather, this sentiment was often expressed in comments that they wished that the class would continue over several terms or that they could stay in school longer. A number of students did, in fact, continue their school year by taking other Women's Studies courses. However, it is unclear how capstone participants will 
maintain this process of growth without a supportive community that allows continued progression in reflective capacities and opportunities for critical action - two key components of continued SPC consciousness development and maintenance identified by Watts and colleagues (2011).

Further, finding a critical community to engage in also provides the emotional energy necessary to transfer reflection into action (Summers-Effler, 2002). Participants stressed the importance of safe spaces, both for themselves and for the girls in the rap sessions, but no participants expressed plans to seek out or create such spaces after leaving the higher education system. This is a not a problem exclusive to Girl Power, but extends to all capstone courses and classes in Critical Pedagogy more broadly. At the time the interviews were being conducted, members of the University Studies community at Portland State University were beginning to talk about and develop strategies to keep students engaged in social change work following the intensive capstone experience and even after graduation.

The idea of safe space was central to many participants. They discussed how both the rap sessions and the course served as a safe space. The idea of safe spaces was important to the second wave of the women's movement in the United States (Ferree \& Hess, 2000). Currently, safe spaces are being used in LGTBQ communities (Fetner, Elafros, Bortolin, \& Drechsler, 2012). Collins (2000) discusses the importance of safe spaces as a realm of relatively sheltered discourse, which she views as a necessary condition for Black women's resistance. She suggests these spaces are not only safe but they form prime locations for resisting objectification as the other. Fetner and colleagues 
(2012) suggests that these spaces have three important areas of safety, context (safe from what?), membership (safe for whom?), and activities (safe for what?). The course functions as a safe space that allows room (figurative and physical) for identity development, sharing a sense of community, and development of critical consciousness. However, the idea of safe spaces is in and of itself problematic. It is important to ask who a space is safe for, and simultaneously who or what is thus unwelcome in that space. While it is important for any safe space to negotiate guidelines for interaction (as is done in the Girl Power activity, co-creating our ideal classroom community) these guidelines cannot guarantee that all members of the space feel welcomed. In fact, by creating guidelines, the values of some groups are held as more appropriate, thus potentially violating the values of other groups. Even when the values of the safe space include important aspects of civil discourse and dialogue, such as mutual respect and openness, it is important to consider how these even seemingly innocuous and positive goals may make some groups feels more comfortable than others.

While joining a critical community or safe space has the potential to maintain and expand critically conscious capacities, if taken too far this can also become limiting. If students were to eschew all other forms of social support except for the critical community, they might be pushed towards fanatic consciousness. Further, such communities have the capacity to become insular or labeled as separatist, thus limiting opportunities for exposure to new ideas and opportunities for creating change in broader society. 


\section{Chapter Seven: Conclusion}

This chapter concludes the dissertation by reflecting on the theoretical implications of the findings of my study, as well as their limitations in transferring beyond the site of the Girl Power capstone course. The implications of the dissertation for future research on SPC consciousness development also are discussed.

\section{Overview of Study}

Methods. This dissertation involved two phases of data collection. First, I underwent a process of preliminary research that involved sitting through one quarterterm of the Girl Power capstone course as a participant observer. Utilizing the findings of that preliminary work and my theoretical framework I developed and conducted interviews with consenting capstone participants from the term I observed. Ten semistructured interviews were conducted, transcribed, and analyzed using the Listening Guide. Following the analysis, two primary themes were identified_processes of awakening and sources of dissonance.

Processes of Awakening. The processes of awakening was a recurring and multidimensional theme throughout the interviews. The material in the processes of awakening theme addressed the first research question: How are emotionally and relationally significant Girl Power experiences related to socio-political-cultural (SPC) consciousness development? The metaphor of "awakening"- as a central themeemerged from descriptors enlisted by participants themselves in characterizing what many viewed as a dramatic learning experience. The theme is described as process because many participants viewed their awakening as an ongoing commitment rather 
than a discrete academic experience based on traditional learning objectives. By combining these two terms, I suggested that the Girl Power course is a part of an ongoing experience of coming to understand the world in a new way. Although participants sometimes described their developing consciousness as a sudden transformation, the general tendency was to describe the "awakening" as a gradual process.

The way in which participants talked about their views and actions before, during, and after Girl Power registered important shifts in reflection, efficacy, and action. Many participants used the language of moving from a place of metaphorical blindness to seeing the world more clearly. Participants noted increased capacity to identify contradictions, deal with ambivalence, observe injustice, think critically, overcome fear, and increased motivation for change, which suggest movement toward critical consciousness. Overall, many participants seemed to believe that they experienced some sort of transformation during or following the Girl Power course. They also tended to view this change as positive. It seemed that a number of structured and unstructured experiences surrounding the course contributed to enhancing SPC consciousness. Important drivers of this process were identified by the four subthemes-the role of Girl Power in the processes of awakening, emotions and the process of awakening, the importance of relationality, and application of the awakening. The first theme addressed the role of the course in participants' experience of transformation. The second and third themes looked specifically at the emotional and relational dynamics that participants identified as playing a role in their changing awareness. The final area addressed participants' behavioral changes that accompanied their changing views of the world. 
Each of these subthemes contributed to the processes of awakening in distinct and overlapping ways.

Overall, the processes of awakening theme seemed to support the dissertation's focus on dialectical consciousness as it stressed bringing previously hidden or unacknowledged thoughts and feelings into awareness. This awareness then brought to light concealed aspects of ideology that maintain oppression (Moane, 2010). Awareness, coupled with the community-oriented nature of the course, allowed students to begin to express emotions and thoughts in advancing a critical analysis of the dominant society. Further, the coupling of awareness, relationships, and emotions allowed participants to shift the blame from individuals to larger social structures. The developing awareness combined with the emotional energy generated in the course grew out of a number of sites of activity, from rituals (e.g., check-ins and check-outs), cultural artifacts (e.g., the Zine), to identification with a charismatic leader (e.g., the instructor) and an intergenerational subculture (e.g., the mentor groups). Each allowed students to develop their level of critical reflection and their capacity for critical efficacy and action. As suggested by Summers-Effler (2002) and laid out in the theoretical framework, each of these areas enhance emotional energy which allows individuals to break free of their dependence on the status quo for fulfillment, allowing room for movement intellectually, emotionally, and behaviorally towards critical consciousness.

The level of reflection developed by the participants did not seem to extend to analyzing oppression at multiple levels. While many participants were adept at discussing and interrupting oppression at the interpersonal level, their analysis of how 
sexism and other forms of oppression functioned at the institutional or systematic levels was limited. This blind spot suggested an area of potential development for future Girl Power courses. In addition, no participants expressed that they expanded their level of awareness on their own in the months following the class. This lack of awareness of oppression at multiple levels may have contributed to a corresponding lack of interest in fighting oppression on these same levels (a key component of critical consciousness). Indeed, participants exclusively relied on interruptions as a critical action strategy.

Sources of Dissonance. The theme of dissonance emerged in relation to the second research question: What tensions arise between the dominant culture and/or significant others' values and the values of the Girl Power capstone and how do these tensions move individuals toward or away from critical consciousness? This question was included because dissonance was hypothesized as an important factor in moving students along the SPC consciousness continuum. This study conceptualizes consciousness as a dialectic process involving internal forces (e.g., drives, cognitions, and emotions), which are dynamically related to the external world (e.g., material reality, culture, and ideology) in an ongoing process of the world acting on consciousness and consciousness (through behaviors) acting on the world (i.e., praxis). Dissonance may move students forward toward critical consciousness or backward toward preconsciousness, depending on how they work with the dissonance. Individuals that are able to deal with ambivalence and dissonance move towards critical consciousness. Those that eschew conflict or cannot tolerate tension are driven backward on the continuum of SPC consciousness. 
Participants experienced multiple sources of dissonance in the course. First, participants experienced internal discord as they struggled to integrate their new understanding of the world with old thoughts and actions. Dissonance in this area often seemed to motivate participants to work for more continuity in their new understanding of the world and the thoughts, actions, and language they deployed on a regular basis.

A second area of tension arose around the Girl Power course itself, including the rap sessions. While the specific areas of tension surrounding the course and the intensity of the tension varied from participant to participant, most experienced some level of conflict during the course. Participants particularly struggled to balance the conflicting demands of the experiential and academic content of the class.

A third area of dissonance was evident in students' interpersonal relationships. As participants began to see the world in different ways through their process of awakening, they struggled with relationships outside of the course. Participants stated that friends, family members, and partners that had previously known them sometimes resisted their new views of the world. The process of interrupting oppressive language of friends and family often exacerbated this conflict. For many participants, this was the area of dissonance that was most salient and problematic.

A final area of dissonance for participants centered on their changing relationships and reactions to recreational activities and mainstream culture. While participants overwhelmingly stated that the new critical lens they developed towards culture was an advance, they also confronted new barriers. Some participants were no longer able to enjoy previously relaxing and fun activities (e.g., reading magazines, going 
dancing, watching television). Along with contributing to dissonance in relationships, this area of tension may be problematic if participants are not able to find new activities to enjoy without guilt. It participants began to feel guilt over everything they watched, read, and listened to, the unpleasant feeling might drive them backward on the SPC consciousness continuum. Individuals are driven to relieve feelings of dissonance because of the psychological discomfort they cause. Changing thoughts, feelings, or behaviors can relieve this tension. While changing a behavior to relieve dissonance can be a long-term solution that prevents further dissonance, this option is difficult because many behaviors are difficult to change. A simpler option for dealing with dissonance involves changing one's thoughts or emotions, which could result in regressive trends in the critical reflection or critical efficacy aspects of SPC consciousness.

Bridging the themes. While the two themes were separate strands in the interview data, the themes were also interconnected, as would be expected utilizing the Listening Guide as a mode of analysis. Themes are dynamically related to one another, sometimes in dissonance and sometimes in harmony. The two primary themes presented in this dissertation seem to support one another. For example, many participants were able to deal with the internal dissonance of falling short of the expectations and ideals developed through the Girl Power course by reminding themselves that awakening is a process - and a very hard process. Overall, the strategy of justifying tension through acknowledging difficulties as part of a process seemed to make areas of dissonance more manageable for participants. In other words, the idea that awakening is a process seemed to help participants deal with many of the sources of conflict they faced during and 
following the Girl Power class. The idea of process allowed participants room to make mistakes, such as slipping up and using an oppressive term they were trying to strike from their vocabulary. Further, the idea of process allowed participants to have patience with conflict in relationships, as they acknowledged that it might take friends and family a while to "catch up" to their awakening.

While the relationship between the two themes was not the focus of the current study, this area of inquiry offers an opportunity for expansion and deepening of the findings presented in this dissertation. Later analysis of the collected interviews with the focus of bridging these two themes might elucidate additional findings regarding the process of SPC consciousness development.

\section{Limitations to the Study}

The limitations and the strengths of this study are intertwined. While the qualitative and context-specific nature of the study allowed me to pursue my interests in processes and a specific locale, it also introduced a number of limitations. One of the restrictions of the study involved the limited life experiences and geographical region of the site of inquiry. All participants were women, living in Portland and college students who were within a few quarters of graduation. Their shared participation in the Girl Power course made their experience highly unique and not necessarily generalizable to other arenas in which critical consciousness development may unfold. Further, I was not able to interview all of the participants in the capstone course. A sample size of ten is acceptable for the methods utilized in this dissertation but the students that decided to 
participate may have been different in their responses from students that chose not to participate.

Other limitations of the study centered on the time frame and resources available. I did not have any funding to conduct this study and I was limited in my ability to engage in more long-term research because of the requirements to finish my doctorial degree in a timely manner. The time frame, lack of funding, and individual nature of the dissertation meant that the number of interviews and courses that could be observed was restricted.

While the applied value of the study must be addressed given the very specific context in which it was conducted, the goal of the dissertation was not generalizability. Instead, this study aimed for transferability. Also, I was interested in gaining insight into the process of critical consciousness development, and particularly in developing an understanding of the emotional and relational components of the process.

Emotions are notoriously difficult to study. Emotions are sometimes fleeting, often conflicting, partially unconscious, and socially constructed. Because of their complexity, individuals are often limited in their capacity to discuss and interpret their own emotions. The very idea of emotions as something that is felt suggests difficulty in describing them in a cognizant way. People often turn to art to express emotions because the bounds of language confound the ability to describe such a complex phenomenon. Given the elusiveness of these phenomena, it is not surprising that at times the participants struggled to articulate the emotions connected to Girl Power and their process of SPC consciousness. In interviews, participants often stammered around finding the words to use for emotions, or they chose words such as happy or frustrated. 
They often seem unsatisfied with the choice of these words, as if these words could not fully capture the range of their experience. While this study does offer a more nuanced look into the emotions involved in critical consciousness development, it is also limited in its understanding of emotions.

The findings related to relationality, more specifically, seemed more clear and prominent than findings related to emotional dimensions of SPC consciousness development. Participants were more open and able to express the impact their changing consciousness had on their relationships as compared to the emotions involved in the process. Many participants were particularly animated and talkative when discussing the role of relationships in their processes of awakening and the subsequent dissonance that their developing SPC consciousness had on their relationships.

The study was primarily situated in a constructivist interpretive paradigm that strove for trustworthiness, credibility, transferability, and confirmability (Denzin \& Lincoln, 2005). The focus on transferability replaced the idea of generalization. To facilitate readers' ability to transfer my findings and conclusions to environments and populations with which they are familiar I have provided detailed description of the settings and the methods utilized in the research (Lincoln \& Guba, 1985).

\section{Contributions and Implications}

This study has sought to expand Martín-Baró's (1994) idea that "the fundamental horizon for psychology as a field of knowledge is concientizacion [critical consciousness]" (p. 39). The importance of addressing critical consciousness as an area of intersection between psychology, education, and social change has also been taken up by 
other community psychologists (see Montero, 2009; Watts et al., 2011). However in a recent literature review on the subject Watts and colleagues (2011) lamented that the understanding of critical consciousness lacked theoretical models and empirical support. The work presented here begins to address this gap in the field by offering a way to understand the relational and emotional aspects of critical consciousness development through the integration of feminist, psychoanalytic, and social movement theories. By utilizing these theoretical tools, this study developed a more nuanced understanding of the role of emotions and relationality in the process of critical consciousness development that adds to the previous focus on the cognitive aspects of the phenomenon. Further, by integrating an understanding of emotions and relationality, this study offered a psychological avenue to understand the disconnection that is often observed between thoughts and actions- specifically, the development of critical reflection that is not accompanied by a subsequent increase in critical action.

The findings of this dissertation sought to offer a context specific understanding of the process of development of SPC consciousness. This focus on contextualized knowledge follows in the tradition of community psychologist such as Trickett (1996) who call for the necessity of context specific knowledge that attends to historical and environmental factors. By applying a dialectic understanding to critical consciousness in a specific setting, I was able to develop a model of critical consciousness that goes beyond conceptualizing the phenomenon as a simple state or a one-way stage model of development. The utility of the model of critical consciousness as the highest state of SPC consciousness offers conceptual and theoretical specificity to the phenomenon that 
was lacking in previous literature. The data collected in this dissertation represents a first step in supporting the theoretical model of critical consciousness development presented in the theoretical framework.

On a practical or applied level, the findings of the dissertation could be used to build better interventions to develop critical consciousness. For those committed to social change the three aspects of critical consciousness- critical reflection, critical efficacy, and critical action- represent three important capacities needed for working towards social justice. SPC consciousness development can occur in a variety of settings. This study focused on its development in a university classroom, which had its own set of specific constraints. Higher education is distinct from other settings that focus on enhancing critical consciousness in that it must be paid for and carried out within a larger bureaucratic structure-one that is typically hierarchical, and includes a formal evaluation (i.e., grades). Further, there are punitive rules associated with dropping a class, such as, receiving a failing grade, losing money, losing financial aid, and delaying graduation because of a loss in credit hours. These issues are particularly salient in the Girl Power course because it was worth six credit hours (double that of most courses). While the classrooms focus on enhancing SPC consciousness often strove to integrate aspects of activist groups into its setting (e.g., decreasing the amount of hierarchy, utilizing participatory decision making, analyzing oppressive structures) it could never fully reproduce those conditions. Understanding the role of the unique setting of the higher education classroom on SPC consciousness development helps to contextualize the psychological knowledge of the critical consciousness development. While the 
importance of emotions and relationships is likely a driving force for the development of critical consciousness in activist circles (see Polletta, 2002), no less than in capstone courses, some areas of dissonance and tension may be different in the two settings. Some forms of psychological dissonance seem potentially unique to the classroom setting, particularly those centered on performance demands and the problem of how to continue a process of SPC consciousness development beyond the temporal framework of a course.

Important parallels could be drawn between the struggles the students face following the completion of Girl Power and those faced by activists when a movement or change organization dissolves. The findings of this dissertation suggest that relationality is a central driving force for SPC consciousness development. It seems that both groups would benefit from an increased understanding of how to develop and maintain spaces that foster opportunities for increasing critical reflection, critical efficacy, and involvement in critical action. While the Girl Power course offers one way of building a space many consider safe, an enhanced understanding of how to construct these spaces and the limitations of these spaces would be beneficial to future work on critical consciousness development and social change more broadly.

This dissertation also has implications for the field of education. The role of emotions in the classroom is heavily debated. Some scholars argue that emotions are essential to the learning process and thus must be integrated into any classroom (Krathwohl, Bloom, \& Masia, 1964). That said, some education practitioners are trained that emotions are the enemy of rationality and are an unpredictable and dangerous force 
within the classroom and should be avoided. Still many scholars and educators suggest that emotions are present in any group setting and ignoring them is problematic (Boler, 1999). This dissertation fell within the theorizing of this last group in arguing that emotions are integral to any process of learning, and particularly where ideals and investments in worldviews are at stake. However, few educators receive training in how to work with emotions that arise in the classroom-a factor that may contribute to teachers' perceptions that emotions can easily get out of control. The findings from this study suggests that if the classroom is to be a setting with the capacity to enhance critical consciousness then it must be recognized as a site of intense feeling and relationality. Educators could benefit from feminist, psychoanalytic, and social movement scholars that bring emotional processes into analyses of social change projects. The role of emotions and the corresponding role of relationality can both drive and inhibit SPC consciousness development (Hoggett, 2009; Summers-Effler, 2002). The findings of this dissertation specifically suggested that emotions such as hope and anxiety seemed to play a role in facilitating critical efficacy that can contribute to critical consciousness. Alternatively, fear seemed to inhibit SPC consciousness development. Harnessing and understanding the role of these and other emotions in the classroom offers the opportunity for the expansion of Critical Pedagogy techniques beyond the purely cognitive level.

It is also important that instructors avoid delving into areas that confuse the classroom with the clinical setting. While emotions exist in the classroom and should be recognized and worked with, it is important to keep the classroom from turning into a group therapy session. While emotionally evocative class time may aid in the process of 
critical consciousness development, if students feel forced to over share in such settings this may become problematic for some individuals. Some participants in this study were perhaps pushed too far beyond their comfort level in the course, as was suggested by two participants suggesting they had to seek outside therapeutic help in dealing with the emotions that arose surrounding the course. The findings from this dissertation suggest that instructors should attend to what constitutes appropriate barriers in emotional expression and sharing in the classroom.

\section{Possibilities for Future Research}

Much of the prior research on Critical Pedagogy has focused on participants' reactions at the completion of an experiential learning experience. There is very little research available, however, on participants' subsequent assessments of that experience. This study sought to address this gap by delaying primary data collection until five months after the completion of the course. However, future research on critical consciousness development might look at multiple points in time following the completion of an intervention. This longitudinal frame might generate better understandings of how participants' work through those areas of dissonance identified in this dissertation. This understanding could help recognize if SPC consciousness development moves both forward and backward as predicted by the theoretical framework or if once a specific level of SPC consciousness has been reached, movement only occurs in a forward direction, as suggested by participants. This knowledge could aid in designing future critical consciousness interventions. 
While the focus of this research was on the process of critical consciousness development, future research might include gathering data before and after the interventions to gain better understanding of changes in critical consciousness as a result of the intervention. Evaluations of this sort might help in gaining a better understanding of the best practices for developing critical consciousness in different settings and populations.

The Girl Power capstone is a particular type of course and experience that requires a high level of investment from students in the classroom and in the community, as well as on the part of the instructor. The capstones, particularly those based on social justice principles such as Girl Power, may include an expectation that students produce a narrative of personal transformation. While this expectation may have invited the processes of awakening that unfolded in Girl Power, it might also have been accompanied by problematic demand characteristics in how students respond to the course, and to my research. It would be important for future research in this area to look at other types of classes to see if these findings hold in a broader range of education settings. Further, the idea of a capstone as an integration of knowledge with a transformational future may, in itself, carry problematic assumptions about higher education and advancement.

The dissertation was carried out in an urban environment that is known as relatively progressive politically. The dissonance that participants face in this environment may be very different from the tensions students experience in a more conservative city or region of the country. Future research might seek to untangle how 
different social, political, cultural, and religious environments affect the types and intensities of dissonance faced by individuals as they develop and attempt to maintain critical consciousness.

Another area of expansion for future research could look into different ways to study the emotional component of SPC consciousness development. As discussed in the limitations section, this dissertation's focus on interviews and observations limited what could be known about complex emotional experiences. Inquiry might involve multiple interviews with participants so that the researcher could probe for deeper and more nuanced emotional experiences. Further, researchers might enlist focus groups to attend to collective processes in the construction of emotions. Additionally, studies might include methodologies that involve art or the use of facial expressions and other nonlingual ways of expressing and understanding emotional components of critical consciousness development.

\section{Conclusion}

This dissertation was situated at the nexus of three areas--psychology, education, and social change. While these areas are often seen as distinct from one another as domains and fields of study, this dissertation has the aim of bridging these three areas and understanding how their convergence contributes to critical inquiry. Psychology can offer important insight into how education and social change are enacted, and education is an important aspect of social change.

In this dissertation, I attempted to contribute to a body of psychological knowledge that can lead to social change efforts. In understanding the processes and 
tensions involved in critical consciousness development, including emotional and relational components, better interventions could be designed to enhance human capacities for critical reflection that is oriented toward critical action and social transformation.

In this research project I have also attempted to connect education to the social change project through the development of engaged citizens envisioned by a long line of scholars working at the intersection of psychology, education, and social change over the course of the past century, from John Dewey, Paulo Freire, Ignacio Martín-Baró to bell hooks, Carol Gilligan, and Michelle Fine. In contributing to this critical tradition of scholarship, I hope to increase the understanding and enhance the practice of fighting oppression at multiple levels and subsequently contribute to the ongoing work of social change. 


\section{References}

Adler, P., \& Adler, P. (1987). The past and the future of ethnography. Journal of Contemporary Ethnography, 16, 1, 4-24.

Albee, G. W. (1978). A competency model to replace the defect model. Paper presented at the Vermont Conference on Primary Prevention.

Armstrong, E. A. (2002). Forging gay identities: Organizing sexuality in San Francisco, 1950-1994. Chicago: University of Chicago Press.

Au, W. (2007). Epistemology of the oppressed: The dialectics of Paulo Freire's theory of knowledge. Journal for Critical Education Policy Studies, 5, 2.

Au, W. (2012). Critical curriculum studies: Education, consciousness, and the politics of knowing. New York: Routledge.

Bartky, S. (2004). On psychological oppression. In L. M. Heldke \& P. O'Connor (Eds.), Oppression, privilege, and resistance: Theoretical perspectives on racism, sexism, and heterosexism. (pp. 24-36). Boston: McGraw-Hill.

Bernstein, M. (1997). Celebration and suppression: The strategic uses of identity by the Lesbian and Gay Movement. American Journal of Sociology, 103, 3, 531-565.

Blumer, H. (1951). Social Movements. In Lee, A. M. C., \& Park, R. E. (Eds.), New outline of the principles of sociology. (pp. 199-220). New York: Barnes \& Noble.

Boler, M. (1997). Disciplined emotions: Philosophies of educated feelings. Educational Theory, 47, 2, 203-227.

Boler, M. (1999). Feeling power: Emotions and education. New York: Routledge.

Brenner, J., \& Haaken, J. (2000). Utopian thought: re-visioning gender, family, and community. Community, Work \& Family, 3, 3, 333-347.

Brown, L. M. (2001). White working-class girls, femininities, and that paradox of resistance. In D. L. Toleman \& M. Brydon-Miller (Eds.), From subjects to subjectives: A handbook of interpretive and participatory methods (pp. 95-110). New York and London: New York University Press.

Brydon-Miller, M. (2001). Education, research, and action: Theory and methods of participatory action research. In D. L. Toleman \& M. Brydon-Miller (Eds.), From subjects to subjectives: A handbook of interpretive and participatory methods (pp. 76-89). New York and London: New York University Press. 
Burton, M., \& Kagan, C. (2005). Liberation social psychology: Learning from Latin America. Journal of Community \& Applied Social Psychology, 15, 1, 63-78.

Carlson, E. D., Engbretson, J., \& Chamberlain, R. M. (2006). Photovoice as a social process of critical consciousness. Qualitative Health Research, 16, 6, 836-852.

Checkoway, B. (2000). Public service: Our new mission. Academe, 86, 4, 24-28.

Cho, D., \& Lewis, T. (2005). The persistent life of oppression: The unconscious, power, and subjectivity. Interchange, 36, 3, 313-329.

Clarke, S., Hoggett, P., \& Thompson, S. (2006). Emotion, politics and society. Basingstoke England: Palgrave Macmillan.

Collins, P. H. (1992). Black feminist thought: Knowledge, consciousness, and the politics of empowerment. New York: London: Routledge.

Cornelius, D. (1998). Walking the walk: Socializing students to social activism. Teaching Sociology, 26, 190-197.

Crenshaw, K. (1991). Mapping the margins: Intersectionality, identity politics, and violence against women of color. Stanford Law Review, 43, 1241-1258.

Crociani-Windland, L., \& Hoggett, P. (2012). Politics and affect. Subjectivity, 5, 2, 161 179.

Cutforth, N., Stoecker, R., Marullo, S., Donohue, P., \& Strand, Kerry J. (2006). Community-Based Research and Higher Education: Principles and Practices. Wiley.

Cudd, A. (2006). Analyzing oppression. New York: Oxford University Press.

Damasio, A. R. (1994). Descartes' error: Emotion, reason, and the human brain. New York: Putnam.

Denzin, N. K., \& Lincoln, Y. S. (2005). Introduction: The discipline and practice of qualitative research. In N. K. Denzin \& Y. S. Lincoln (Eds.), The SAGE handbook of qualitative research (pp. 1-32). Thousand Oaks: SAGE Publications.

Dewey, J. (1999). Democracy and education: An introduction to the philosophy of education. New York: Free Press. 
Diemer, M., \& Blustein, D. (2007). Vocational hope and vocational identity: Urban adolescents career development. Journal of Career Assessment, 15, 1, 98-118.

Diemer, M., Hsieh, C., \& Pan, T. (2009). School and parental influences on sociopolitical development among poor adolescents of color. The Counseling Psychologist, 37, 2, 317-344.

Diemer, M. A., \& Li, C. H. (2011). Critical consciousness development and political participation among marginalized youth. Child Development, 82, 6, 1815-1833.

Diemer, M. A., Wang, Q., Gregory, S. R., Hatcher, K. M., Voight, A. M., \& Moore, T. (2010). Sociopolitical development, work salience, and vocational expectations among low socioeconomic status African American, Latin American, and Asian American Youth. Developmental Psychology, 46, 3, 619-635.

Doucet, A., \& Mauthner, N. S. (2008). What can be known and how? Narrated subjects and the listening guide. Qualitative Research, 8, 3, 399-409.

Erikson, E. H. (1968). Identity, youth, and crisis. New York: W. W. Norton.

Eyler, J. (2002). Reflection: Linking service and learning—linking students and communities. Journal of Social Issues, 58, 3, 517-534.

Fanon, F. (1967). Black skin, white masks. New York: Grove Press.

Ferree, M. M., \& Hess, B. B. (2000). Controversy and coalition: The new feminist movement across three decades of change. New York: Routledge.

Festinger, L., Riecken, H. W., \& Schachter, S. (1956). When prophecy fails. Minneapolis: University of Minnesota Press.

Fetner, T., Elafros, A., Bortolin, S., \& Drechsler, C. (2012). Safe spaces: gay-straight alliances in high schools. Canadian Review of Sociology $=$ Revue Canadienne De Sociologie, 49, 2, 188- 207.

Fine, M., Torre, M. E., Boudin, K., Brown, I., Clark, J., Hylton, D., et al. (2003). Participatory action research: From within and beyond prison bars. In P. M. Camic, J. E. Rhodes \& L. Yardley (Eds.), Qualitative research in psychology: Expanding perspectives in methodology and design. Washington, DC: American Psychological Association.

Fine, M., \& Vanderslice, V. (1992). Qualitative activist research: Reflections on methods and politics. New York: Plenum. 
Freeman, J. (1973). The origins of the women's liberation movement. American Journal of Sociology, 78, 4, 792-811.

Freire, P. (1993). Education for critical consciousness. New York: Continuum.

Freire, P. (2000). Pedagogy of the oppressed. New York: Continuum.

Fromm, E. (1994). Escape from freedom. New York: H. Holt

Gamson, W. A. (1990). The strategy of social protest. Belmont, Calif: Wadsworth Pub

Gamson, W. A. (1992). Talking politics. Cambridge England: Cambridge University Press.

Gemignani, M. (2011). Between Researcher and Researched: An Introduction to Countertransference in Qualitative Inquiry. Qualitative Inquiry, 17, 8, 701-708.

Gilligan, C. (1982). In a different voice: Psychological theory and women's development. Cambridge, Mass: Harvard University Press

Gilligan, C., Spencer, R., Weinberg, M. K., \& Bertsch, T. (2003). On the listening guide: A voice-centered relational method. In S. N. Hesse-Biber \& P. Leavy (Eds.), Emergent methods in social research (pp. 253-271). Thousand Oaks, CA: SAGE Publications.

Guest, G., Bunce, A. \& Johnson, L. (2006) How many interviews are enough? An experiment with data saturation and variability. Field Methods, 18, 1, 59-82.

Guishard, M. (2009). The false paths, the endless labors, the turns now this way and Now that: Participatory Action Research, mutual vulnerability, and the politics of inquiry. The Urban Review, 41, 1, 85-105.

Haaken, J. (1998). Pillar of salt: Gender, memory, and the perils of looking back. New Brunswick, N.J: Rutgers University Press.

Haaken, J. (2008). When White Buffalo Calf Woman meets Oedipus on the road: Psychoanalysis, Lakota psychology and male violence. Theory \& Psychology, 18, 195-208.

Haaken, J., Wallin-Ruschman, J., \& Patange, S. (2012). Global hip-hop identities: Black youth, psychoanalytic action research, and the Moving to the Beat project. Journal of Community and Applied Social Psychology, 22, 1, 63-74. 
Hochschild, A. R. (1983). The managed heart: Commercialization of human feeling. Berkeley: University of California Press.

Hoggett, P. (2009). Politics, identity, and emotion. Boulder, CO: Paradigm Publishers.

hooks, b. (1994). Teaching to transgress: Education as the practice of freedom. New York: Routledge.

Ife, J. (2001). Human rights and social work: Towards rights-based practice. Cambridge: Cambridge University Press.

Jasper, J. M. (2002). Review essay: What the devil are emotions?. Qualitative Sociology, $25,1,145-148$.

Jasper, J. M. (2011). Emotions and social movements: Twenty years of theory and research. Annual Review of Sociology, 37, 285-303. Publications.

Kelly, A. (1971). Qualities for the community psychologist. American Psychologist, 26, 897-903.

Kidder, L. H., \& Fine, M. (1997). Qualitative inquiry in psychology: A radical tradition. In D. Fox and I. Prilleltensky (Eds.), Critical psychology: An introduction (pp. 34-50). Thousand Oaks, CA: SAGE Publications.

Kohlberg, L. (2008). The development of children's orientations toward a moral order: Sequence in the development of moral thought. Human Development, 51, 8-20

Krathwohl, D. R., Bloom, B. S., \& Masia, B. B. (1964). Taxonomy of educational objectives: The classification of educational goals. Handbook 2: Affective domain. New York: McKay.

Landreman, L. M., Rasmussen, C. J., King, P. M., \& Jiang, C. X. (2007). A phenomenological study of the development of university educators' critical consciousness. Journal of College Student Development, 48, 3, 275-296.

Lincoln, Y. S., \& Guba, E. G. (1985). Naturalistic inquiry. Beverly Hills, Calif: Sage Publications.

Lincoln, Y. S., \& Guba, E. G. (2005). Paradigmatic controversies, contradictions and emerging confluences. In N. K. Denzin \& Y. S. Lincoln (Eds.), The SAGE handbook of qualitative research (pp. 191-215). Thousand Oaks, CA: SAGE 
Lykes, M. B. (1989). Dialogue with Guatemalan Indian women: Critical perspectives on constructing collaborative research. In R. K. Unger (Ed.), Representations: Social constructions of gender (pp. 167-185). Amityville, NY: Baywood Publishing Co.

Lynn, M., Benigno, G., Williams, A., Park, G., \& Mitchell, C. (2006). Critical theories of race, class, and gender in urban education. Encounter: Education for meaning and social justice, 19, 2, 17-25.

Martín-Baró, I., Aron, A., \& Corne, S. (1994). Writings for a liberation psychology. Cambridge, Mass: Harvard University Press.

Marx, K. (1978). Critique of the Hegelian dialectic and philosophy as a whole. In R. C. Tucker (Ed.), The Marx-Engels reader (pp. 106-125). New York: W. W. Norton.

Moane, G. (2010). Sociopolitical development and political activism: Synergies between feminist and liberation psychology. Psychology of Women Quarterly, 34, 4, 521529.

Montero, M., \& Sonn, C. C. (2009). Psychology of liberation: Theory and applications. New York: Springer.

Mustakova-Possardt, E. (1998). Critical consciousness: An alternative pathway for positive personal and social development. Journal of Adult Development, 5, 1, 13 30.

Mustakova-Possardt, E. (2003). Critical consciousness: A study of morality in global, historical context. Westport, Conn: Praeger.

Mustakova-Possardt, E. (2004). Education for critical moral consciousness. Journal of Moral Education, 33, 3, 245-269.

Nelson, G. B., \& Prilleltensky, I. (2010). Community psychology: In pursuit of liberation and well-being. Basingstoke, UK: Palgrave Macmillan.

Passini, S., \& Morselli, D. (2011). In the name of democracy: Disobedience and valueoriented citizenship. Journal of Community and Applied Social Psychology, 21, 3, 255-267.

Patton, M. Q. (2001). Qualitative research \& evaluation methods. Thousand Oaks, Calif: Sage Publications.

Piaget, J. (1953). The origin of intelligence in the child. London: Routledge \& Paul. 
Pitner, R. O., \& Sakamoto, I. (2005). The role of critical consciousness in multicultural practice: Examining how its strength becomes its limitation. The American Journal of Orthopsychiatry, 75, 4, 684-94.

Piven, F. F., \& Cloward, R. A. (1979). Poor people's movements: Why they succeed, how they fail. New York: Vintage books.

Polletta, F. (2002). Freedom is an endless meeting: Democracy in American social movements. Chicago: University of Chicago Press.

Prilleltensky, I. (1989). Psychology and the status quo. American Psychologist, 44, 5, 795-802.

Prilleltensky, I. (2001). Value-based praxis in community psychology: Moving toward social justice and social action. American Journal of Community Psychology, 29, $5,747-778$

Prilleltensky, I. (2008). The role of power in wellness, oppression, and liberation: The promise of psychopolitical validity. Journal of Community Psychology, 36, 2, 116-136.

Prilleltensky, I., \& Gonick, L. (1996). Polities change, oppression remains: On the psychology and politics of oppression. Political Psychology, 17, 1, 127-148.

Prilleltensky, I., \& Nelson, G. (1997). Community psychology: Reclaiming social justice. In D. Fox \& I. Prilleltensky (Eds.), Critical psychology, pp. 166-184. Thousand Oaks, CA: Sage.

Rappaport, J. (1977). Community psychology: Values, research and action. New York: Holt, Rinehart \& Winston.

Rappaport, J. (1987). Terms of empowerment/exemplars of prevention: Toward a theory for community psychology. American Journal of Community Psychology, 15, 2, 121-48.

Rappaport, J. (1998). The art of social change: Community narratives as resources for individual and collective identity. In X.B. Arriaga \& S. Oskamp (Eds.), Addressing community problems: Psychological research and interventions, (pp. 225-246). Thousand Oaks, CA: Sage.

Reason, R., Scales, T., \& Roosa, M. E. (2005). Encouraging the development of racial justice allies. New Directions for Student Services, 110, 55- 66. 
Rhodes, T. L., \& Agre-Kippenhan, S. (2004). A Multiplicity of learning: Capstones at Portland State University. Assessment Update, 16, 1, 4-6.

Riger, S. (1993). What's wrong with empowerment? American Journal of Community Psychology, 21, 279-292.

Ryan, W. (1971). Blaming the victim. New York : Vintage Books.

Serrano-Garcia, I. (1994). The ethics of the powerful and the power of ethics. American Journal of Community Psychology, 22, 1-20.

Smelser, N. J. (1963). Theory of collective behavior. New York: Free Press of Glencoe.

Smith, J.A. (1995). Semi-structured interviewing and qualitative analysis. In J.A. Smith, R. Harre \& L. Van Langenhove (Eds.), Rethinking methods in psychology (pp. 926). Thousand Oaks, CA: SAGE Publications.

Smith, W. A. (1976). The meaning of conscientizacao: The goal of Paulo Freire's pedagogy. Amherst, Mass: Center for International Education, University of Massachusetts.

Snow, D.L, Grady, K., \& Goyette-Ewing, M. (2002). A perspective on ethical issues in community psychology. In J. Rappaport \& E. Seidman (Eds.), Handbook of Community Psychology. (pp. 897-917). New York: Kluwer/Plenum.

Staggenborg, S. (2011). Social movements. New York: Oxford University Press.

Stein, C.H. \& Mankowski, E.S. (2004). Asking, witnessing, interpreting, and knowing: The process of conducting qualitative research in community psychology. American Journal of Community Psychology, 33, 3/4, 21-35.

Stiles, W. B. (1993). Quality control in qualitative research. Clinical Psychology Review, $13,6,593-618$.

Stokamer, S. T., \& Portland State University. (2011). Pedagogical catalysts of civic competence: The development of a critical epistemological model for communitybased learning. Portland, Or.: Portland State University.

Summers-Effler, E. (2002). The micro potential for social change: Emotion, consciousness, and social movement formation. Sociological Theory, 20, 1, 4160. 
Tolman, D. L., \& Brydon-Miller, M. (2001). From subjects to subjectivities: A handbook of interpretive and participatory methods. New York: New York University Press.

Trickett, E. J. (1998). Toward a framework for defining and resolving ethical issues in the protection of communities involved in primary prevention projects. Ethics \& Behavior, 8, 4, 321-337.

Valenzuela, A. (2005). Subtractive schooling, caring relations and social capital in the schooling of U.S.-Mexican youth. In L. Weis \& M. Fine (Eds.), Beyond silenced voices: Class, race, and gender in United States schools (pp. 83-94). New York: SUNY Press.

Wachtel, P. L. (2011). Therapeutic communication: Knowing what to say when. New York: Guilford Press.

Wallerstein, N., \& Sanchez-Merki, V. (1994). Freirian praxis in health education: Research results from an adolescent prevention program. Health Education Research, 9, 1, 105-18.

Watts, R. J., \& Abdul-Adil, J. K. (1998). Promoting critical consciousness in young, African-American men. Journal of Prevention and Intervention in the Community, 16, 63-86.

Watts, R. J., Abdul-Adil, J. K., \& Pratt, T. (2002). Enhancing critical consciousness in young African American men: A psychoeducational approach. Psychology of Men and Masculinity, 3, 40, 41-50.

Watts, R. J., Diemer, M. A., \& Voight, A. M. (2011). Critical consciousness: current status and future directions. New Directions for Child and Adolescent Development, 2011, 134, 43-57.

Watts, R. J., \& Flanagan, C. (2007). Pushing the envelope on youth civic engagement: A developmental and liberation psychology perspective. Journal of Community Psychology, 35, 6, 779-792.

Watts, R. J., Griffith, D. M., \& Abdul-Adil, J. (1999). Sociopolitical development as an antidote for oppression-theory and action. American Journal of Community Psychology, 27, 2, 255-271.

Watts, R., \& Guessous, O. (2006). Sociopolitical development: The missing link in research and policy on adolescents. In S. Ginwright, P. Noguera, \& J. Cammarota (Eds.), Beyond Resistance! Youth Activism and Community Change: New 
Democratic Possibilities for Practice and Policy for America's Youth. (pp. 59-80). NY: Routledge.

Watts, R. J., Williams, N. C., \& Jagers, R. J. (2003). Sociopolitical Development. American Journal of Community Psychology, 31, 1-2.

Wallin-Ruschman, J., \& Portland State University. (2011). The moving to the beat documentary and hip-hop based curriculum guide: Youth reactions and resistance. Portland, Or: Portland State University.

Young, I. M. (1990). Justice and the politics of difference. Princeton, N.J: Princeton University Press. 


\section{Appendix A: Interview Guide}

1. When you think back on the course, what stands out?

a. Why do you think that is?

b. Do you think other students had a similar experience? Why or why not?

2. Did you bring in any artifacts (e.g., Zine pages, reflection papers, art) from the course?

If yes, can you show them to me and tell me about them?

a. Why did you bring these particular artifacts?

b. What do these artifacts mean to you?

c. How do these artifacts make you feel?

d. Have you produced any artifacts like this post-Girl Power?

If not, think of the artifacts you produced from the course that were most meaningful.

a. What would you have brought, and why?

b. What do these artifacts mean to you?

c. How do these artifacts make you feel?

d. Have you produced any artifacts like this post-Girl Power?

3. Here is a timeline of significant events that occurred throughout the course. [after offering a chance to review the timeline]

a. Could you talk about how you felt about the course at the beginning?

Could you tie these feelings to specific course experiences?

i. In the middle?

ii. In the end?

iii. When you think back on these experiences now, what are your feelings now?

iv. Do you think other students had a similar overall experience? Why or Why not?

b. Were any of the events on the timeline particularly hard or challenging? How so?

i. Were there times during the course that you felt conflicted about something? What?

ii. Do you think other students experienced similar conflicts? Why or why not?

4. When discussing the goals of Girl Power the instructor often says, "it is approaching the cultural lie in a courageous way." 
a. What does this statement mean to you?

b. Has your understanding of this phrase changed over time? If yes, how so?

5. How would you classify your level of feminist or social justice consciousness before the course? Immediately following the completion of the course? Now?

a. If changes: To what do you attribute these changes?

b. Do you think your level of social justice consciousness will stay the same? Why or why not?

c. If changes: Have you experienced any change in behavior connected to your change in social justice consciousness?

a. If no changes: Did anything change as a result of your Girl Power experience?

Below I start the more specific questions. I may skip these if they have already been addressed.

6. What was your overall mood in the Girl Power capstone? Did this fluctuate over time?

a. What was your overall level of emotional energy in the course? Did this fluctuate over time?

7. Has your group of friends or community changed following Girl Power? How so?

a. Do you hang out with any of the people you met in Girl Power?

8. Has your reaction to dominant culture (e.g., movies, media, music) changed following Girl Power? How so?

9. Overall, do you think Girl Power works in creating feminist and/or social justice consciousness? Why or why not?

a. What aspects of the course seem most central to success (or failure)?

b. What could be done to make it more effective at this task?

10. Before the capstone did you ever feel that you experienced emotions that were 'wrong' or considered 'unacceptable'?

a. If yes, what were these emotions?

b. Why do you think you felt this way about these emotions?

c. Did these feelings change (either the emotions themselves or how you felt about them) change during or after Girl Power?

11. I know this is a big questions but could you tell me what you think are the roots of social injustice?

a. Is this something that changed over you Girl Power experience?

b. To what do you attribute these changes? 
c. In your experience, what do you think are the best methods to promote social justice?

12. Did you experience any tension between what you were learning and feeling in Girl Power and other aspects of your life (e.g., internal, family, religion, media, other classes)? How so?

a. If yes: could you describe this tension?

b. Did you resolve this tension? How?

13. Was there any part of your Girl Power experience that was significant that we have not discussed? What? Could you tell me more about it? Why was it significant? 


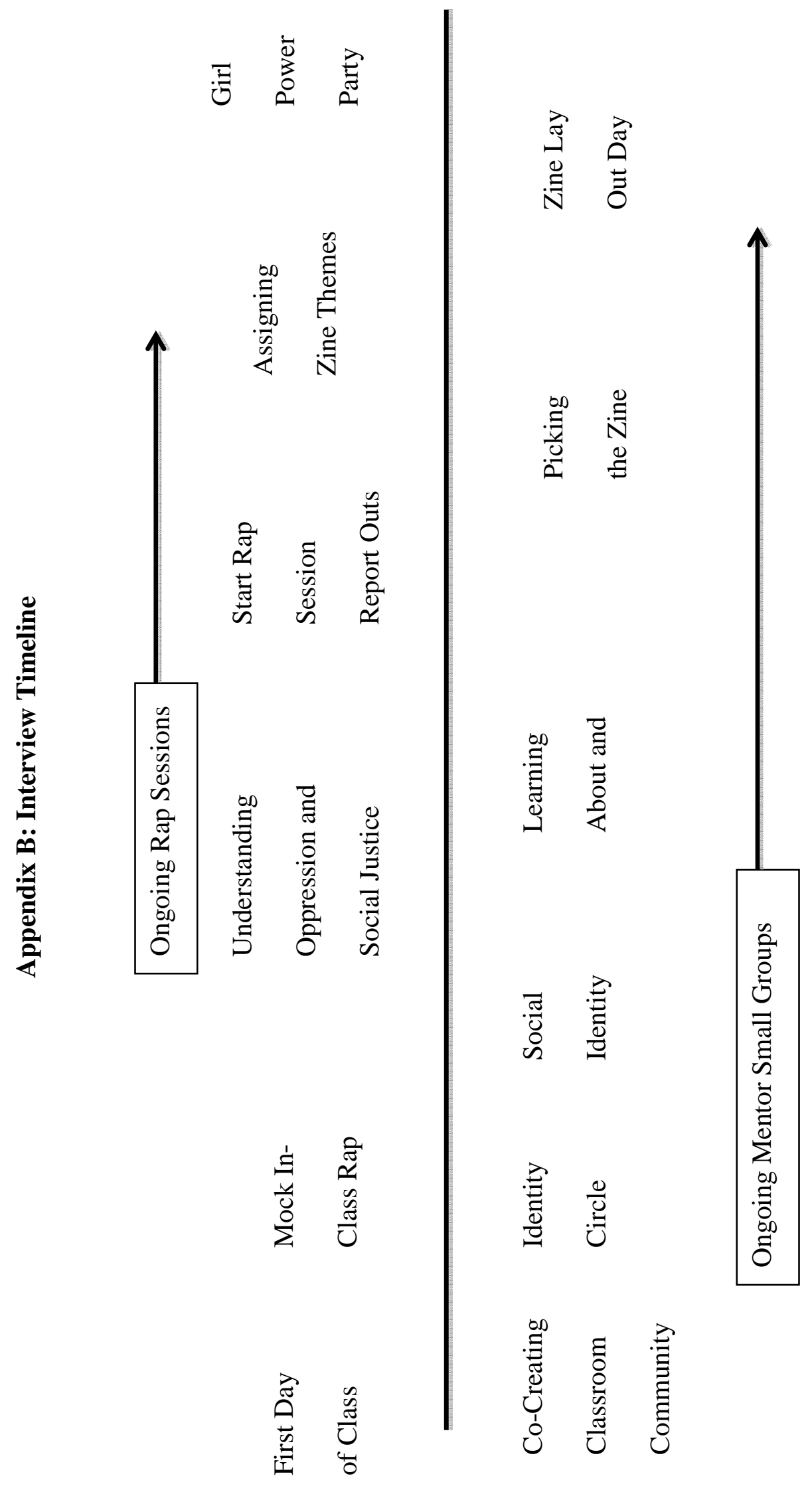




\section{Appendix C: Smith's Conscientization Coding Categories Outline}

$\underline{\text { Magical Consciousness }}$

A. Naming

a. Problem Denial

i. Overt denial

ii. Problem avoidance

b. Survival Problems

i. Poor physical state/health

ii. Poverty

iii. Lack of work

iv. Insufficient work

v. Money as end in itself

B. Reflecting

a. Simplistic Causal Relationships

i. Blames physical state/health

ii. Blames objects over people

b. Facts Attributed to Superior Powers

i. Uncontrollable factors: God/Fate/luck/Age/etc.

ii. Fear of oppressor

iii. Oppressor as inevitable winner

C. Acting

iv. Empathy for oppressor

a. Fatalism

i. Resignation

ii. Acceptance

b. Passively Playing Host to Oppressor

i. Waiting for "good:" luck/patron

ii. Dependence on oppressor

\section{Naïve Consciousness}

A. Naming

a. Oppressed Deviates from Ideal Expectations

i. Oppressed not like oppressor/oppressed not meet oppressor's expectations

B. Reflecting

ii. Horizontal aggressiveness/intrapunitiveness

a. Blames Oppressed

i. Accepts oppressor's expectations (education as end in itself)

ii. Self-peer deprecation

iii. Blames ancestors 
iv. Self-pity

b. Understands How Individual Oppressor Violates Norms

i. Sees intentionality by oppressor

ii. Sees relationships between oppressor/oppressor's agents

iii. Generalizes from one individual oppressor to another.

C. Acting

a. Actively Playing Host to Oppressor (Collusion)

i. Models oppressor's behavior (education, dress, habits)

ii. Misdirected aggression (horizontal aggression/intrapunitiveness)

iii. Paternalistic towards peers

iv. Meets oppressors expectations

b. Defending

i. Gregariousness

ii. Makes system work

iii. Avoids oppressor

iv. Opposes individual oppressor

v. Change environment

Critical Consciousness

A. Naming

a. Rejection of Oppressor Groups/Self-Peer Affirmation

i. Rejects oppressors groups

ii. Seeks to maintain ethnicity

iii. Seeks to affirm uniqueness

b. Transforms System

i. Procedures-People

B. Reflecting

ii. Rejects oppressive system

a. Understands/Rejects Oppressors' Ideology and Their Collusion

i. Sympathy/understanding peers

ii. Self-critical/sees contradiction between actions and critical goals

iii. Rejects horizontal aggression/affirms self

iv. Sees oppressor as weak/victim of system

v. Rejects oppressor/oppressor's ideology

vi. Generalizes from one oppressive group to another

b. Understands How System Works

i. Sees systems as cause

ii. Sees contradictions between rhetoric/results

iii. Macro-socio-economic analysis

iv. Generalizes from one oppressive system to another

C. Acting 
a. Self-Actualization

i. Sees appropriate role models

ii. Personal/ethnic self-esteem

iii. Self-growth /transforming learning

iv. Subject/author

v. Faith in peers/peer learning

vi. Boldness/risk-taking/unorthodox solutions

vii. Reliance on community resources/participation

viii. Opposes oppressor groups

b. Transforming System

i. Dialogue-polemics

ii. Comradeship

iii. Scientific approach

iv. Change norms/laws/procedures

From:

Smith, W. A. (1976). The meaning of conscientizacao: The goal of Paulo Freire's pedagogy. Amherst, Mass: Center for International Education, University of Massachusetts, p. 72-74. 


\section{Appendix D: Watts and Abdul-Adil Five Stage Model of Sociopolitical Development}

1. Acritical Stage: Asymmetry is not subject to critical thought and the capacity of critical consciousness is limited. The person may see inequity as the natural way of the world; that is, it is a "just world" and those with low status deserve it because they are inferior. Internalized oppression (thoughts and feelings of inferiority) can help sustain feelings of powerlessness or inferiority.

2. Adaptive Stage: The person may acknowledge inequity, but the system maintaining it is seen as immutable. Predatory, anti-social or accommodation strategies are employed to maintain a positive sense of self and to acquire social and material rewards. Critical consciousness does not contribute to an understanding of the long-term sociopolitical consequences of adaptive behavior or its role in maintaining oppression.

3. Pre-Critical Stage: Complacency gives way to a critical awareness of, and concerns about asymmetry and inequality. The value of adaptation is questioned.

4. Critical Stage: Critical consciousness skills undergo their most rapid development and use. Motivation is highest for learning about asymmetry by the analysis of the social and historical roots of asymmetry, injustice, and oppression. Critical consciousness will lead some people to conclude that the asymmetry is unjust, and that social-change efforts are needed. The person uses Adaptive strategies less frequently.

5. Liberation Stage: The experience and awareness of oppression are salient. Involvement in social action and community development is tangible and frequent. Adaptive behavior is eschewed. Critical consciousness is an established component of self.

From:

Watts, R. J., \& Abdul-Adil, J. K. (1998). Promoting Critical Consciousness in Young, African-American Men. Journal of Prevention and Intervention in the Community, 16, 63-86. 


\section{WHAT I LIKEABCUT BEINGA GIILL}

Tooth fairy: I like being able to (guys don't really match their outfits), but women can... we have more options than them

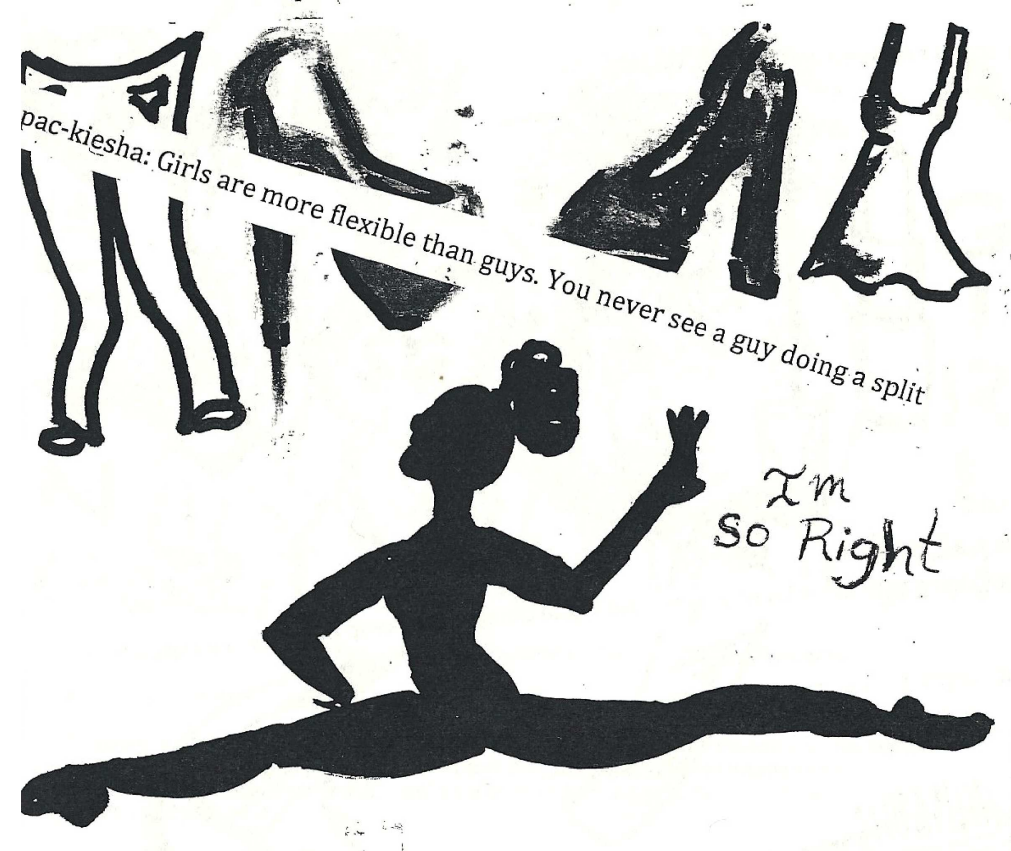

Ieeh: you can show guys that they're not boss and you can prove them wrong 


\title{
Appendix F: Informed Consent Document
}

\author{
A Girl Power Study: Looking and Listening to the Role of Emotions in \\ Developing Critical Consciousness
}

You are invited to participate in a research study conducted by graduate student researcher Jen Wallin-Ruschman under the direction of Jan Haaken $\mathrm{PhD}$ at Portland State University (PSU). The purpose of this consent form is to give you the information you will need to help you decide whether or not to be in the study. Please read this form carefully. You may ask questions about the purpose of the research, what I will ask you in the interview, the possible risks and benefits, your rights as a volunteer, and anything else about the research or this form. When all your questions have been answered, you can decide if you want to be in the study.

\section{Purpose of the Study}

The researchers hope to learn about the role of emotions in feminist and social justice consciousness and the role of the Girl Power capstone in increasing feminist and social justice consciousness. This study is being conducted in partial fulfillment for a doctoral degree and is under the supervision of Jan Haaken. You were selected as a possible participant in this study because you were a student in the Girl Power capstone course in Fall 2012.

\section{$\underline{\text { Procedures }}$}

If you agree to participate, you will be asked to be interviewed for one to two hours about your emotional experiences in Girl Power and your feminist and social justice consciousness. The interviews will take place at Portland State University. With your permission, I would like to audio record your interview so that I can have an accurate record. The audio recording will be saved on a password protected computer. You will have the opportunity to pick the alias, see below, I will use to identify you in the transcribed interview and materials based on the information you provide in your interview. If you choose to pick an alias that could be linked to your identity confidentiality can no longer be guaranteed. Please indicate below whether or not you give your permission for me to audio record your interview. If you do not give permission to audio record your interview, I will take notes during the interview to document your responses. Only my advisers, trained research assistants, and I will have access to the research materials (e.g., audio recording, transcriptions).

\section{Possible Risks}

Although it is unlikely, there is a chance that you may experience discomfort in talking about your feelings and/or Girl Power experience. You will have the chance to discuss their feelings during the interview but may also choose not to share these thoughts and 
feelings. You can choose to not answer any questions and you can decide to stop at any time. If you decide to stop your participation, you will not face any negative repercussions, and I will not be offended or upset.

\section{Potential Benefits}

You may feel good talking about your experiences and sharing your story. The information we collect in this study will help us to improve and understand the education practices and themes related to social justice and feminist consciousness. Information also will be the basis of a graduate student dissertation project, and potentially papers published through academic journals.

\section{Participant Statement}

Your participation is voluntary and may be terminated at any time. If you do not wish to participate it will not affect your relationship with Girl Power, or Portland State University, or the researchers.

This study has been explained to me. I volunteer to participate in this research. I have had a chance to ask questions. If I have questions later on about the research, I can ask the investigator, Jen Wallin-Ruschman by calling her at (270) 792-7906, emailing her at jlwallin@pdx.edu, or writing to her at P.O. Box 751, Department of Psychology, Portland State University, Portland, OR 97207-0751.

If I have questions about my rights as a research participant, I can contact the Portland State University Human Subjects Research Review Committee at (503) 725-4288, (877) 480-4400, by mail at Portland State University, Market Center Building, 6th Floor, P.O. Box 751, Portland, OR 97207-0751, or by email at hsrrc@pdx.edu.

If I have questions for Jen Wallin-Ruschman's adviser, I can contact Jan Haaken, PhD at (503) 725-3967, by mail at Psychology Department, 317 Cramer Hall, Portland State University, P.O. Box 751 Portland, OR 97207-0751, or by email at haakenj@pdx.edu.

I have received a copy of this consent form.

\section{Audio Recording}

I give my permission for the researcher to audio record my interview.

I do not give my permission for the researcher to audio record my interview.

\section{Consent}


I give my consent to be interviewed.

Participant Printed Name

Participant Signature

Date

Investigator Signature

Date

Alias

The alias I choose to identify my information for this project is: 